Portland State University

PDXScholar

Winter 3-24-2014

\title{
Adjustment to College among Lower Division Students with Disabilities: An Exploratory Study
}

Kristy Lee Ann McNulty

Portland State University

Follow this and additional works at: https://pdxscholar.library.pdx.edu/open_access_etds

Part of the Disability and Equity in Education Commons

Let us know how access to this document benefits you.

\section{Recommended Citation}

McNulty, Kristy Lee Ann, "Adjustment to College among Lower Division Students with Disabilities: An Exploratory Study" (2014). Dissertations and Theses. Paper 1653.

https://doi.org/10.15760/etd.1652

This Dissertation is brought to you for free and open access. It has been accepted for inclusion in Dissertations and Theses by an authorized administrator of PDXScholar. Please contact us if we can make this document more accessible: pdxscholar@pdx.edu. 
Adjustment to College among Lower Division Students with Disabilities: An Exploratory Study

by

Kristy Lee Ann McNulty

A dissertation submitted in partial fulfillment of the requirements for the degree of

\author{
Doctor of Education \\ in
}

Educational Leadership: Special and Counselor Education

\author{
Dissertation Committee: \\ Hanoch Livneh, Chair \\ Russ Miars \\ Susan Halverson-Westerberg \\ Laurie Powers
}

Portland State University

2014 
(C) 2014 Kristy Lee Ann McNulty 


\begin{abstract}
This study utilized a quality of life framework of psychosocial adaptation to explore relationships among college stress, functional limitations, coping strategies (engagement-type and disengagement-type coping), and perceived social support in adjustment to college among first-year and second-year undergraduate students with disabilities, based on specific hypothesized relations. College adjustment outcomes included: life satisfaction, academic performance, and psychosocial-emotional adjustment to college.
\end{abstract}

A nonprobability sample of 103 first-year and second-year undergraduate college students with disabilities participated in the study. Respondents were registered with an office of support services for students with disabilities at a public, four-year university, located in the Pacific Northwest of the United States. Respondents were recruited by responding to an e-mail requesting participation in an online, web-based survey.

Eight self-report measures included: (a) Participant Survey (developed by the researcher to collect socio-demographic information), (b) College Stress Inventory (CSI; Solberg, Hale, Villarreal, \& Kavanagh, 1993), (c) Disability Functional Limitations Scale (DFLS) (developed by the researcher), (d) Brief COPE; Carver, 1997, (e) Social Support Appraisals-Revised (SSA-R) scale; Vaux et al., 1986), (f) Satisfaction with Life Scale (SWLS; Diener, Emmons, Larsen, \& Griffin, 1985), (g) Grade Point Average-Scale (GPA-S; adapted by the researcher from a self-reported grading scale], and (h) Student Adaptation to College Questionnaire (SACQ; Baker \& 
Siryk, 1999).

Data were analyzed using descriptive and correlational procedures. Bivariate analysis suggested that all predictor variables (i.e., college stress, functional limitations, engagement coping, and perceived social support) were significantly associated with student adjustment to college. Hierarchical multiple regression suggested mostly direct (i.e., main) effects for engagement coping and perceived social support. No interacting role for either engagement-type coping or perceived social support was suggested, except for the following: Engagement-type coping moderated the relationship between disability-related functional limitations (as measured by increased restrictions in the ability to perform daily routines, activities, and life roles) and adaptation to college, as measured by life satisfaction. Analyses of socio-demographic variables revealed significant associations between chronological age, gender, hours employed, and adjustment to college. Lastly, hierarchical multiple regression analysis revealed disengagement coping accounting for as much as $53 \%$ of the variance in adjustment scores. This result suggested disengagement coping adding significant predictive utility for adaptation-associated college adjustment.

In light of these findings, counseling professionals may wish to consider the beneficial role of engagement coping in promoting optimal adjustment to college for lower-division undergraduate students with disabilities. 


\section{Dedication}

This dissertation is dedicated to my mother, Milly McNulty, brother, Jack McNulty, and nephew, Alan McNulty. I especially appreciated their support and assistance with computer-related crashes and other serious computer-related hardware and software problems occurring throughout the entire dissertation process. 


\section{Acknowledgements}

To my advisor, Hanoch Livneh Ph.D., I cannot express enough gratitude and thanks for your guidance and support throughout the entire dissertation process. A special thank you also goes to Paula Stanovich, Ph.D. who served on my dissertation committee until her unexpected retirement in 2011. Paula Stanovich Ph.D. provided valuable insights and contributions to this project from her perspective as a professional in the field of special education. I would also like to especially recognize and thank Russ Miars, Ph.D., for his assistance in coordinating my dissertation credits during summer and fall terms, 2013. It was also an honor and pleasure to work in the Master's counseling clinic with Russ Miars, Ph.D. A special thanks is also extended to Susan Halverson-Westerberg Ph.D., and Laurie Powers Ph.D., who served as members of my dissertation committee. 


\section{Table of Contents}

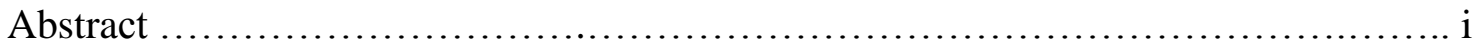

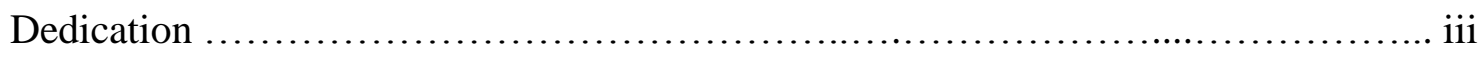

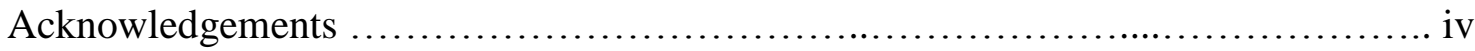

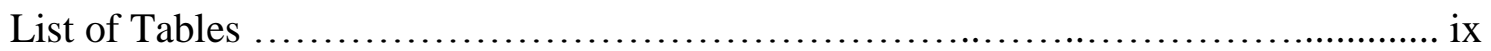

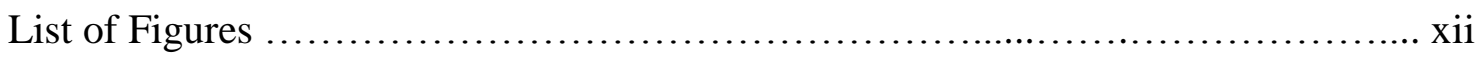

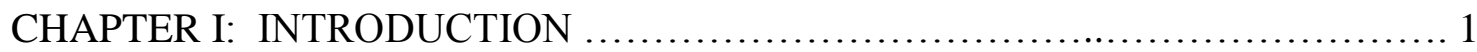

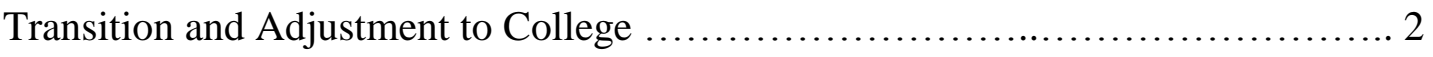

Students with Disabilities and Adjustment to College $\ldots \ldots \ldots \ldots \ldots \ldots \ldots \ldots \ldots \ldots \ldots 5$

The Concept of Functionality and Adjustment to College ........................ 6

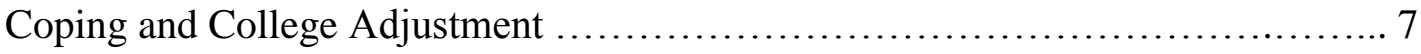

Perceived Social Support and College Adjustment ............................ 12

Adaptation-Associated Outcomes: A Quality-of-Life Framework ................ 17

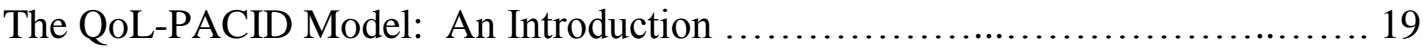

Intrapersonal Coping \& Adjustment to College .............................. 22

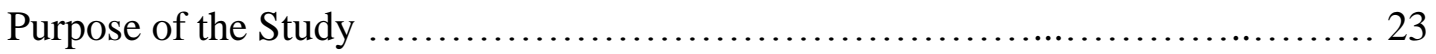

Study Aims, Hypotheses, and Research Questions ............................ 25

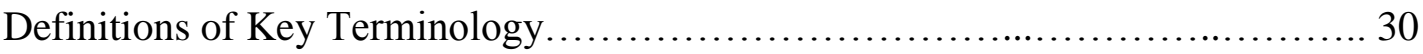

CHAPTER II: REVIEW OF THE LITERATURE ............................ 32

Overview: Students with Disabilities and Adjustment to College .................. 33

Frameworks of Adaptation: A Rehabilitation Perspective ........................ 39 


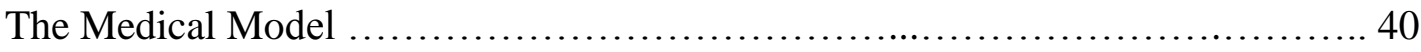

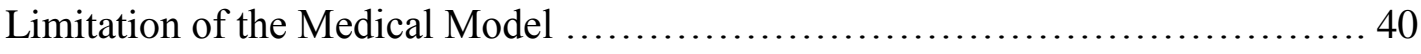

Stage/Phase Models .................................................. 41

Limitations of Stage/Phase Models ....................................... 44

Ecological Models ..................................................... 46

Limitations of Ecological Models: The Process of Adaptation ..................... 53

Limitations of Ecological Models: Outcomes of Adaptation Process .............. 54

Theoretical Consensus................................................. 55

Quality-of-Life-Based Model of Adaptation .................................. 57

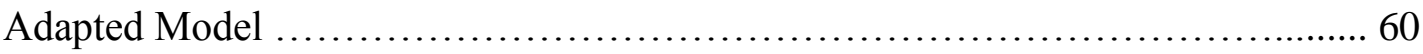

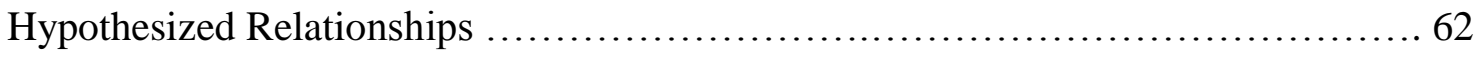

CHAPTER III: METHODOLOGY ......................................... 79

Research Aims/Statistical Analysis ....................................... 79

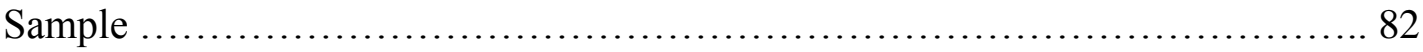

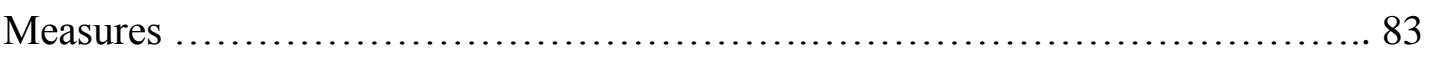

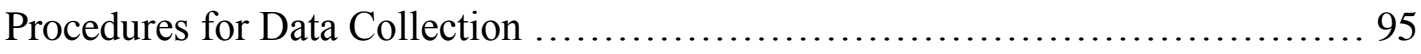

CHAPTER IV: DATA ANALYSIS ........................................ 97

Preparation of Data for Analysis ......................................... 98

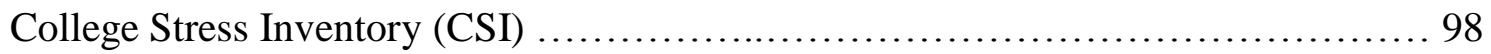

Disability Functional Limitations Scale (DFLS) .............................. 99

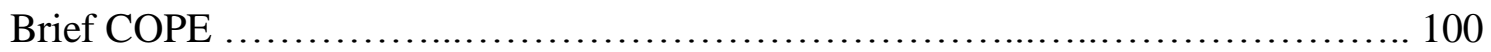


Social Support Appraisals-Revised Scale (SSA-R) ............................ 102

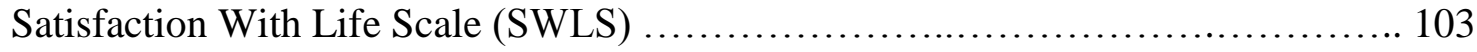

Grade Point Average-Scale (GPA-S) ...................................... 104

Student Adaptation to College Questionnaire (SACQ) .............................. 105

Results of Quantitative Analysis ........................................ 107

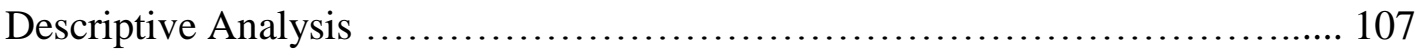

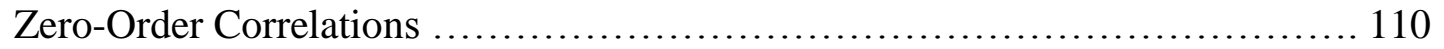

Testing Bivariate Relationships: Hypotheses 1-4............................. 113

Testing Moderating (Interactive) Relationships: Hypotheses 5-8 ............... 122

Exploratory Research Questions ........................................ 156

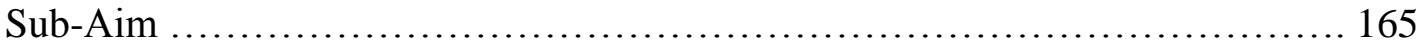

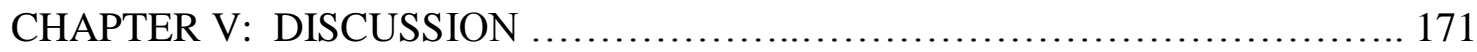

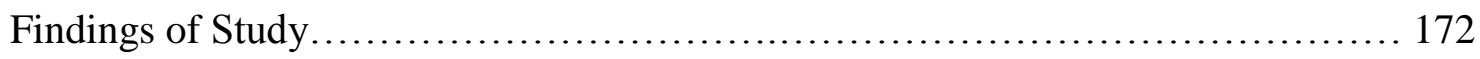

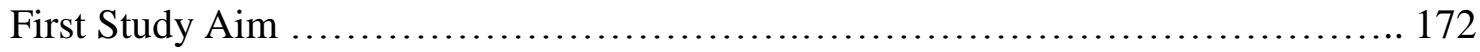

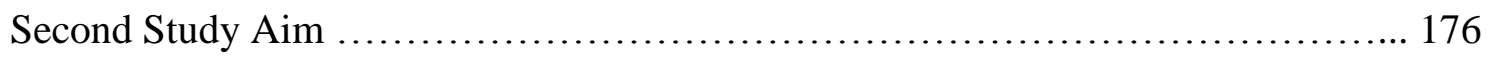

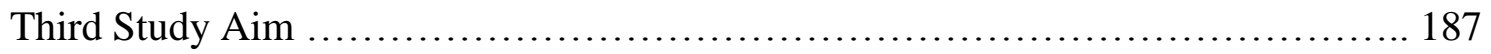

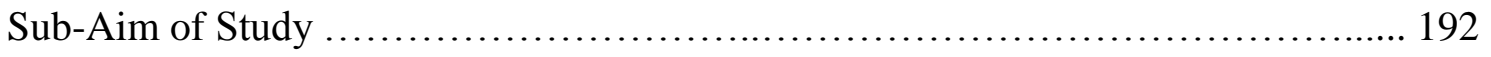

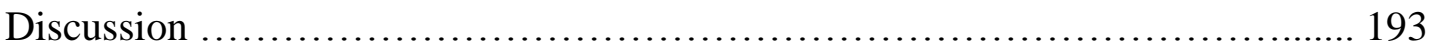

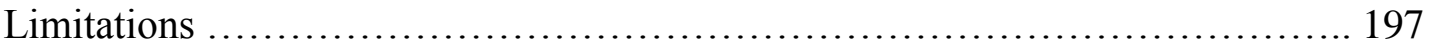

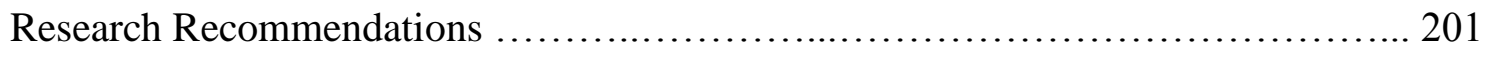

Implications for Rehabilitation Practice ....................................... 202 


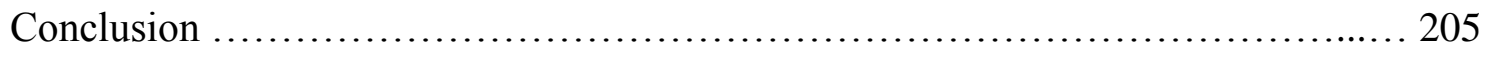

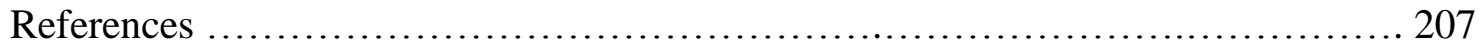

APPENDIX A: Participant Questionnaire ......................................... 260

APPENDIX B: College Stress Inventory (CSI) ................................. 265

APPENDIX C: Disability Functional Limitations Scale (DFLS) …............... 267

APPENDIX D: Social Support Appraisals-Revised Scale (SSA-R) ................. 268

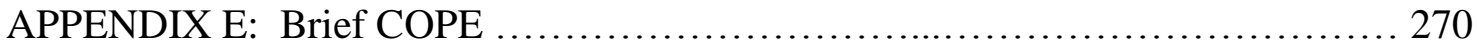

APPENDIX F: Satisfaction With Life Sale (SWLS) .............................. 273 


\section{List of Tables}

Table

Page

1. Means and Standard Deviations for Predictor and Outcome Variables

112

2. Zero-Order Correlations for Predictor and Outcome Adjustment Variables

3. Correlations among Predictor (CSI) and Outcome Measures (SWLS, GPA-S, SACQ-T) in Sample $(N=103)$ of First-Year and Second-Year College Students with CID

4. Correlations among Predictor (DFLS) and Outcome Measures (SWLS, GPA-S, SACQ-T) in Sample $(N=103)$ of First-Year and Second-Year College Students with CID

5. Correlations among Predictor (E-COPE) and Outcome Measures (SWLS, GPA-S, SACQ-T) in Sample $(N=103)$ of First-Year and Second-Year College Students with CID

6. Correlations among Predictor (SSA-R) and Outcome Measures (SWLS, GPA-S, SACQ-T) in Sample $(N=103)$ of First-Year and Second-Year College Students with CID

7. Hierarchical MRA for Moderating Effects of Engagement Coping on the Predictors (College Stress, Engagement Coping) as measured by Life Satisfaction: An Interaction Model

8. Hierarchical MRA for Moderating Effects of Engagement Coping on Predictors (College Stress, Engagement Coping) as measured by Academic Performance: An Interaction Model

9. Hierarchical MRA for Moderating Effects of Coping on the Predictors (College Stress, Engagement Coping) as measured by Psychosocial-Emotional Adjustment to College: An Interaction Model

10. Hierarchical MRA for Moderating Effects of Coping on the Predictors 135 (Functional Limitations, Engagement Coping) as measured by Life Satisfaction: An Interaction Model 
11. Hierarchical MRA for Moderating Effects of Coping on the Predictors (Functional Limitations, Engagement Coping) as measured by Academic Performance: An Interaction Model

12. Hierarchical MRA for Moderating Effects of Coping on the Predictors (Functional Limitations, Engagement Coping) as measured by Psychosocial-Emotional Adjustment to College: An Interaction Model

13. Hierarchical MRA for Moderating Effects of Perceived Social Support on the Predictors (College Stress, Perceived Social Support) as Measured by Life Satisfaction: An Interaction Model

14. Hierarchical MRA for Moderating Effects of Perceived Social Support on the Predictors (College Stress, Perceived Social Support) as Measured by Academic Performance: An Interaction Model

15. Hierarchical MRA for Moderating Effects of Perceived Social Support on the Predictors (College Stress, Perceived Social Support) as Measured by Psychosocial-Emotional Adjustment to College: An Interaction Model

16. Hierarchical MRA for Moderating Effects of Perceived Social Support on the Predictors (Functional Limitations, Perceived Social Support) as Measured by Life Satisfaction: An Interaction Model

17. Hierarchical MRA for Moderating Effects of Perceived Social Support on the Predictors (Functional Limitations, Perceived Social Support) as Measured by Academic Performance: An Interaction Model

18. Hierarchical MRA for Moderating Effects of Perceived Social Support on the Predictors (Functional Limitations, Perceived Social Support) as Measured by Psychosocial-Emotional Adjustment to College: An Interaction Model

19. Correlations among Predictor (DOD) and Outcome Measures (SWLS, GPA-S, SACQ-T) in Sample $(N=103)$ of First-Year and Second-Year College Students with CID

20. Correlations among Predictor (Chronological Age) and Outcome 161 Measures (SWLS, GPA-S, SACQ-T) in Sample $(N=103)$ of First-Year and Second-Year College Students with CID 
21. Correlations among Predictor (Hours Employed) and Outcome Measures 164 (SWLS, GPA-S, SACQ-T) in Sample $(N=102)$ of First-Year and Second-Year College Students with CID

22. Hierarchical MRA of Disengagement Coping (D-COPE) on Life Satisfaction (SWLS) in the Sample $(N=103)$ of First-Year and Second-Year College Students with CID

23. Hierarchical MRA of Disengagement Coping (D-COPE) on Academic Performance (GPA-S) in the Sample $(N=103)$ of First-Year and Second-Year College Students with CID

24. Hierarchical MRA of Disengagement Coping (D-COPE) on

Psychosocial-Emotional Adjustment (SACQ-T) in the Sample $(N=103)$ of First-Year and Second-Year College Students with CID 


\section{List of Figures}

Figure $\quad$ Page

1. Interaction Effects of DFLS and E-COPE on Life Satisfaction (SWLS) 133 


\section{Chapter I}

\section{Introduction}

The current study used a quality-of-life framework of adaptation to explore predictors of college adjustment among lower division undergraduate students with disabilities. Four specific predictor variables included: college stress (college-related characteristic), functional limitations (disability-related characteristic), coping (engagement-type; disengagement-type [intrapersonal characteristics]), and perceived social support (intrapersonal characteristic). The study's adaptation-associated quality-of-life outcomes were measured by three college-related indices of adjustment that included: life satisfaction, academic performance, and psychosocial-emotional adjustment to college.

The primary purpose of the study was to investigate the role of engagement coping and perceived social support (each) as moderating variables that may influence adaptation-associated college adjustment (as measured by life satisfaction, academic performance, and psychosocial-emotional adjustment to college). An empirical exploration of these specific intrapersonal coping strategies may be helpful in guiding rehabilitation professionals to the selection of therapeutic interventions that will

promote optimal adjustment to college for those individuals with disabilities choosing a vocation or career requiring a postsecondary degree.

This chapter begins with a brief discussion on adaptation to college in the general student population, which includes an identification of several college-related factors 
that many undergraduate students face as they transition into college. This is followed by a brief overview of pertinent disability-related factors most individuals with CID deal with on a daily basis, and that may add to the challenges and demands of college life for students with disabilities. One significant disability-related characteristic, and focus of the current study pertains to the concept of functionality. Functionality is defined and discussed, along with its relevance to this study. This is followed by a brief discussion of engagement-type coping and perceived social support as important intrapersonal coping strategies that may influence (un)successful adaptation to college, to include their potential role as moderators in the stress-adjustment relation. Also highlighted in this chapter is the concept of adaptation from a rehabilitation psychology perspective, especially as it pertains to quality-of-life, and the quality-of-life model chosen as the framework to guide the current study. The purpose of the study will then be discussed, followed by the proposed research hypotheses and research questions. Lastly, pertinent terminology used in this study is listed and defined.

\section{Transition and Adjustment to College}

Transitions have been defined as "periods of change, disequilibrium, and internal conflict about gains and losses that occur between periods of stability, balance, and relative quiescence" (Cowan, 1991, p. 7). Such life changes, while often positive in many ways, may also bring with them increased levels of stress as the individual struggles to regain stability in the face of new challenges. One specific life change in American society that has attracted considerable research attention involves students' 
transition to college (Lenz, 2001; Schlossberg, 1981). Although entry into college yields many opportunities for personal growth and development (Arnett, 2000), the literature reveals that students, particularly during their first two years of undergraduate study (Hamilton \& Hamilton, 2006), face a number of potential college-related stressors as they adjust to their new environment (e.g., Baker \& Siryk, 1984; Dyson \& Renk, 2006; Dziegielewski, Turnage, \& Roest-Marti, 2004; Edwards, Herschberger, Russell, \& Market, 2001; Gerdes \& Mallinckrodt, 1994; Herrington, Matheny, Curlette, McCarthy, \& Penick, 2005; Hickman, Bartholomae, \& McKenry, 2000; Kadison \& DiGeronimo, 2004; Kerr, Johnson, Gans, \& Krumrine, 2004; Kitzrow, 2003; Tinto, 1987, 1993; Zivin, Eisenberg, Gollust, \& Golberstein, 2009). Stress may arise from academic factors (e.g., maintaining high academic standards, competition for grades, text anxiety, time management) (e.g., Appelhans \& Schmeck, 2002; Gerdes \& Mallikrodt, 1994; Misra, 2000; Misra \& Castillo, 2004; Mortenson, 2006; Trockel, Barners, \& Egget, 2000) financial pressures (e.g., student debt, credit-card debt) (e.g., Fram \& Bonvillian, 2001; Harding, 2011; Kim, Chatterjee, \& Kim, 2012; Nelson, Lust, Story, \& Ehlinger, 2008) or interpersonal factors (e.g., fluxes in perceived supportive interpersonal relationships due to relocation) (e.g., Calvete \& Connor-Smith, 2006; Coffman \& Gilligan, 2002; Edwards et al., 2001; Friedlander, Reid, Shupak, \& Cribbie, 2007; Gloria \& Ho, 2003; Hickman et al., 2000; Hinderlie \& Kenny, 2002; Misra \& Castillo, 2004; Morosanu, Handley, \& O’Donovan, 2010; Pritchard \& Wilson, 2003; Wintre \& Yaffe, 2000). On-campus counseling professionals have noted that the level of perceived stress 
experienced by college students has grown significantly over the past thirty years, particularly among lower division undergraduate students (Pritchard, Wilson, \& Yamnitz, 2007). The importance of exploring college stress (i.e., as a predictor variable) is related to the recognition by counseling and education professionals that excessive student stress may lead to a negative outcome, such as poor adjustment (e.g., low grade point average [GPA], excessive drinking, depression, anxiety (e.g., Allgower, Wardle, \& Steptoe, 2001; Allgower, Wardle, \& Steptoe, 2001; Constantine, Wilton, \& Caldwell, 2003; Dyson \& Renk, 2006; Kadison \& DeGeronimo, 2004; Misra \& McKean, 2000; Pierceall \& Keim, 2007; Zea, Jarama, \& Bianchi, 1995), or even withdrawal from a college or university (Baker \& Siryk, 1984; Hamilton \& Hamilton, 2006; Tinto, 1993; Zivin et al., 2009).

Despite the importance of a college education for increased earnings, meaningful employment, and subsequent quality-of-life (Carnevale \& Desrochers, 2003), nearly one in four undergraduate students leave college before completing their second academic year (Hamiton \& Hamilton, 2006). First-year (i.e., freshmen-level) and second-year (i.e., sophomore-level) undergraduate students are therefore, most at-risk for college-related adjustment problems. This has prompted counseling and education professionals to focus attention on the research subject of stress, coping, and college adjustment, particularly among first-year and second-year undergraduate students (e.g., Baker, 2003; Pierceall \& Keim, 2007; Saber, Mohmoud, Staten, Hall, \& Lennie, 2012; Sharkin, 2004). 


\section{Students with Disabilities and Adjustment to College}

Because periods of transition are inherently stressful (Cowan, 1991; Lenz, 2001; Schlossberg, 1981), it seems reasonable, therefore, to conclude that the transition to college may be more challenging for those concurrently dealing with other major life demands, such as students with varying chronic illness and disability conditions. Indeed, the rehabilitation psychology and education literatures reveal notable difficulties in adjustment to a postsecondary setting among individuals with varied physical impairments (e.g., epilepsy, multiple sclerosis, paraplegia, diabetes, deafness, blindness, visual/hearing [e.g., Adams \& Proctor, 2010; Elliott, Herrick, \& Witty, 1992; Wodka \& Barakat, 2007; Yagodich, Wolfe, \& Boone, 2000]), cognitive/neurocognitive conditions (e.g., dyslexia, Aspergers, attention deficit hyperactivity disorder [ADHD] [e.g., Blase et al., 2009; Carroll \& Iles, 2006; Gregg, 2007; Gregg et al., 2005; Kaminski, Turnock, Rosen, \& Laster, 2006; Lovett \& Lewandowski, 2006; Milsom, 2007; Milsom \& Hartley, 2005; Neilson, 2001; Nickolas, Menchetti, \& Nettles, 2005; Norwalk, Norvilitis, \& MacLean, 2009; Norvillitis, Sun, \& Zhang, 2009; Shaw-Zirt, Popali-Lehane, \& Chaplin, 2005; Skinner \& Lindstrom, 2003; Sparks \& Lovett, 2009; Weyandt \& DuPaul, 2006]) and psychiatric disabilities (e.g., bipolar disorder, anxiety, depression, post-traumatic stress disorder, schizophrenia [e.g., Breslau, Lane, Sampson, \& Kessler, 2008; Hysenbegasi, Hass, \& Rowland, 2005; Kerr et al., 2004; Megivern, Pellerito, \& Mowbray, 2003; Zivin et al., 2009]). Unfortunately, the role of disability-related factors as possibly influencing adjustment to college among students 
with disabilities remains largely unexplored in the empirical literature. The current study addressed this gap in the literature by including a focus on disability-related variables that may be linked to adaptation-associated college adjustment outcomes in first-year and second-year undergraduate students with disabilities.

\section{The Concept of Functionality and Adjustment to College}

According to the rehabilitation psychology literature (Falvo, 1999; Livneh \& Antonak, 1997, 2005), individuals with chronic illness and disability (i.e., CID) normally face an increase in both the frequency and severity of stressful situations. Among the more commonly recognized disability-related factors that may interact to create increased demands on the lives of individuals with CID include: interference or limitations regarding functional ability, prolonged course of medical or psychiatric treatment, dealing with medication side-effects, and financial concerns involving the cost of health insurance and health care) (Falvo, 1999). In particular, functional aspects of CID's (also termed functionality) have gained increasing recognition among rehabilitation psychology researchers as an important disability-related predictor of psychosocial adaptive outcomes (Livneh \& Wilson, 2003). Functionality has been described in terms of what an individual can and cannot do in relation to the performance of everyday activities and life roles (Eide \& Roysamb, 2002; Heinemann \& Mallinson, 2010; Livneh \& Wilson, 2003; Wineman, 1990; Wineman, Durand, \& Steiner, 1994), and is typically assessed by degree of functional limitations observed or reported (Schalock, 2004). 
Studies on adjustment to CID indicate that severe impediment with functionality (as measured by increased limitations in the ability to perform usual daily tasks and roles) is often associated with poorer psychosocial outcomes (e.g., depression, psychosocial distress, decreased sense of purpose in life) (Dunn, 1996; Eide \& Roysamb, 2002; Ferington, 1986; Haden, Scarpa, Jones, \& Ollendick, 2007; Wineman, 1990; Wineman et al., 1994). For example, in a longitudinal study conducted by the research team of Eide and Roysamb (2002), it was found that functional limitations, as reported by respondents with a wide range of disabling conditions (e.g., emotional, respiratory, musculoskeletal, circulatory) predicted psychosocial adjustment problems over a period of several years. No empirical studies could be located from the rehabilitation psychology and/or education research literatures however, that examined the role of functionality (i.e., more severe restrictions in the ability to perform daily tasks and life roles) as possibly linked to adaptation-associated college adjustment among students with CIDs. Thus, the current study included an exploration of the functional aspects of disability as possibly related to college adjustment outcomes in lower division students with disabilities.

\section{Coping and College Adjustment}

When faced with the transition to a postsecondary setting, new undergraduate students will inevitably have to draw upon coping to deal with their stressful experiences. Indeed, psychosocial adaptation to stressful life conditions and/or crisis situations invariably intimates the existence of personal coping (Livneh \& Wilson, 
2003). As such, coping has increasingly assumed a dominant role in the empirical literature in investigating psychosocial adaptation, to include adaptation to college (e.g., Calvete \& Connor-Smith, 2006; Dyson \& Renk, 2006; Edwards \& Trimble, 1992; Endler, Kantor, \& Parker, 1994; Julal, 2012; Kariv \& Heiman, 2005; Leong et al., 1997; Pieceall \& Keim, 2007; Struthers et al., 2000).

Coping can be viewed as a personal-internal (i.e., intrapersonal) resource that may be mobilized in an effort to decrease, modify, or diffuse the impact of stress-generating life events and/or crisis situations (Billings \& Moos, 1981; Lazarus \&Folkman, 1984). It is defined as "a person's constantly changing cognitive and behavioral efforts to manage specific external and/or internal demands that are appraised as taxing or exceeding the resources of the person" (Lazarus \& Folkman, 1984, p. 141). The individual's coping responses or strategies, are therefore important in sustaining overall functioning during a stressful person-environment interaction (Lazarus \& Folkman, 1984).

Traditionally, coping strategies have been dichotomized into problem-focused coping strategies, or making attempts to actively alter a problematic situation, and emotion-focused coping strategies, or managing emotional responses to a problematic situation (Lazarus \& Folkman, 1984). An alternative empirical categorization of coping has been identified by Tobin, Holroyd, Reynolds, and Wigal (1989): Engagement vs. disengagement. Engagement-type coping strategies refer to those efforts directed at defusing stressful situations through active, goal-oriented activities. Foremost among 
the engagement-type coping efforts are problem focusing, information seeking, seeking social support, active planning, positive reinterpreting/reappraisal, and confronting the source of the problem. In contrast, disengagement coping refers to those strategies that seek to minimize the impact of the stressful situation through passive, indirect, and escape-like efforts. Included are efforts such as denial, wishful thinking, blaming self or others, using alcohol and/or other drugs, social withdrawal, and avoidance (Tobin et al., 1989).

The two dimensions of coping modes, engagement-type and disengagement-type strategies, have been related to different adaptive outcomes in postsecondary student samples, with engagement-type coping efforts positively associated with academic and psychosocial-emotional adjustment (e.g., Appelhans \& Schmeck, 2002; Calvete \& Connor-Smith, 2006; Cosden \& McNamara, 1997; Crockett et al., 2007; Friedlander et al., 2007; Kariv \& Heiman, 2005; Pierceall \& Keim, 2007), and disengagement-type coping efforts associated with poorer overall academic and psychosocial-emotional college adjustment (e.g., Appelhans \& Schmeck, 2002; Aspinwall \& Taylor, 1992; Dyson \& Renk, 2006; Leong et al., 1997; Pierceall \& Keim, 2007; Wodka \& Barakat, 2007).

Engagement-type and disengagement-type strategies, have also been perceived as occupying specific roles within the process of adaptation to life stressors (Livneh \& Wilson, 2003; McNulty, Livneh, \& Wilson, 2004). Two identified roles, also the focus of the current study are (a) predictors of (un)successful adaptation to college (i.e., a 
direct relationship is found between a predictor variable [i.e., engagement-type coping] and various outcome measures), and (b) moderators of the impact of the predictor on the outcome variable, such that the moderator (e.g., engagement-type coping) interacts with the predictor variable to exert a unique impact on the level of the outcome variable (e.g., severity of predictor variable [i.e., college stress; functional limitations] will have less influence on psychosocial adaptation to college when engagement-focused coping is adopted).

Coping as a predictor of adaptation. The empirical literature supports active, engagement-type coping strategies (i.e., one's ability to alter the problematic person-environment relationship causing distress) as most effective in managing stress, and promoting positive adaptive outcomes in postsecondary student samples (e.g., Aspinwall \& Taylor, 1992; Edwards \& Trimble,1992; Endler et al., 1994; Julal, 2012; Kariv \& Heiman, 2005; Leong et al., 1997; Lightsey \& Hulsey, 2002; Misra \& McKean, 2000; Yum, Kember, \& Siaw, 2005). These investigations have included both behavioral (e.g., active planning, seeking social support, information seeking) and cognitive (e.g., cognitive restructuring, reinterpreting/appraising) aspects of engagement-type coping efforts. Furthermore, passive, disengaged coping strategies (the denial of problems; emotional venting; mental and behavioral disengagement) have been associated with poorer outcomes (e.g., Calvete \& Connor-Smith, 2006; Dyson \& Renk, 2006; Heiman \& Kariv, 2004; Pierceall \& Keim, 2007; Wodka \& Barakat, 2007). Only a paucity of studies however, have investigated coping strategies as a predictor of 
(un)successful college adjustment outcomes among students with disabilities.

Unfortunately, these studies have been limited by their relatively small sample sizes, limiting statistical power in testing the association between coping and adaptation-associated outcomes (Heiman \& Kariv, 2004; Sanders \& DuBois, 1996; Wodka \& Barakat, 2007).

Coping as a moderator of adaptation. In classic stress and coping theory, coping strategies have played a critical role in the stress-adjustment relation (Lazarus \& Folkman, 1984). The moderator model is based on the premise that an interaction between stress and the resource (i.e., engagement-type coping) will result in a significant attenuation of negative outcome(s) as a result of higher levels of the resource (Wheaton, 1985). As an intrapersonal strategy, coping has included constantly changing cognitive or behavioral efforts employed to manage situations appraised as taxing or exceeding the resources of a person (Lazarus \& Folkman, 1984). Thus, coping has been viewed as a regulatory process that can reduce or modify the negative feelings resulting from stressful events, particularly when high levels of stress are faced. Empirical investigations support the role of coping strategies operating as a moderator, protecting the individual against the negative impact of stressful episodes when high levels of stress are faced (e.g., Crockett et al., 2007; Haden et al., 2007; Lazarus \& Folkman, 1984; Lee, 2007; Terry, 1989; Wheaton, 1985).

Findings suggest that the engagement dimension (i.e., problem-focused; approach-oriented) of intrapersonal coping may be particularly effective in mitigating 
adverse outcomes under high levels of stress (e.g., Aldwin \& Revenson, 1987; Billings \& Moos, 1981; Crockett et al., 2007; Haden et al., 2007; Lazarus \& Folkman, 1984; Lee, 2007; Olff, Brosschot, \& Godsert, 1993; Pearlin, Menaghan, Lieberman, \& Mullan., 1981; Taylor \& Aspinwall, 1996; Terry, 1989; Wheaton, 1985). The stress interacting effects of engagement-type coping efforts have been documented in studies of the general college student population (e.g., Haden et al., 2007; Hovantz \& Kozora, 1989; Lee, 2007), as well as in ethnically diverse groups, such as African-American and Latino students (e.g., Calvete \& Connor-Smith, 2006; Crockett et al., 2007; Zea, Jarma, \& Bianchi, 1995). On the basis of these studies, engagement-type (i.e., problem-focused forms) coping strategies are suggested as effective in mitigating adverse outcomes under high levels of stress. Unfortunately, no studies could be located in the literature examining the role of coping as a moderator of college-related (i.e., college stress) and/or disability-related (i.e., functional limitations) variables that may be considered stressful, in adjustment to college among students with disabilities. Importantly, the current study sought to address this gap in the literature by exploring the potential moderating role of engagement-type coping in college adjustment among first-year and second-year undergraduate students with disabilities, based on specific hypothesized relationships.

\section{Perceived Social Support and College Adjustment}

The construct of social support has received considerable research attention as a valuable resource influencing (un)successful psychosocial adaptation (Sarason \& 
Sarason, 2009), including adaptation-associated outcomes related to college adjustment (e.g., Calvete \& Connor-Smith, 2006; Coffman \& Gilligan, 2002; Cohen \& Hoberman, 1983; Constantine et al., 2003; Demakis \& McAdams, 1994; Hinderlie \& Kenny, 2002; Mattanah et al., 2010; Mortenson, 2006; Murray, Lombardi, Bender, \& Gerdes, 2012; Swift \& Wright, 2000; Wilks \& Spivey, 2010; Wodka \& Barakat, 2007; Yalcin, 2011; Zea et al., 1995). Conceptually, researchers have distinguished between two extensive types of social support: structural and functional. Structural support refers to quantitative aspects of support such as network size, whereas functional support refers to the qualitative aspects of relationships; that is, the extent to which support is available to an individual (Sarason \& Sarason, 2009; Wills \& Fegan, 2001).

An extensive body of research has delineated the various typologies of social support functions (e.g., Cohen \& Wills, 1985; Hutchinson, 1999), including esteem support (e.g., affect, trust, understanding, value, love, concern, listening, comforting), informational support (e.g., directives, advising, suggestions, information, problem-solving), appraisal support (e.g., social comparison, affirmation, feedback, evaluation), and instrumental support (e.g., material resources, aid-in-kind, tangible assistance). When social support has been conceptualized as the perception of esteem support, also commonly referred to in the literature as perceived social support (Cohen \& Wills, 1985; Thoits, 1982, 1995), it appears to be a much stronger influence in reducing the effects of stress experiences during times of negative life circumstances than any other component or aspect of the social support construct (e.g., Cohen \& Wills, 
1985; Cropley \& Steptoe, 2005; Holahan \& Moos, 1981; Mitchell, Billings, \& Moos, 1983; Thoits, 1995). Perceived social support has also emerged in the empirical literature as a prominent concept that characterizes social support as the cognitive appraisal of being reliably connected to others (Barrera, 1986). It is defined in the current study as: "the belief or perception that one is cared for and loved, esteemed and valued, and a member of a network of mutual support" (Cobb, 1976, p. 26). This definition of perceived social support also fits cognitive models of stress and coping processes that emphasize the appraisal of potentially threatening situations, and intrapersonal strategies that can be enlisted in coping efforts (e.g., Folkman, Schaefer, \& Lazarus, 1979; Lazarus \& Launier, 1978; Moos \& Holahan, 2007; Moos \& Schaefer, 1984, 1993; Wheaton, 1985).

Research on perceived social support has highlighted its role as (a) predictor, and (b) moderator variables influencing (un)successful college adjustment outcomes (e.g., Calvete \& Connor-Smith, 2006; Coffman \& Gilligan, 2002; Solberg \& Villarreal, 1997; Swift \& Wright, 2000; Winterowd, Street, \& Boswell, 1998; Wodka \& Barakat, 2007).

Perceived social support as predictor. Among postsecondary student populations in general, the empirical literature reveals beneficial aspects of perceived social support as a predictor of successful college adaptation; measured for example, by such outcomes as: life satisfaction (e.g., Chao, 2011; Demarkis \& McAdams, 1994; Yalcin, 2011), lower levels of depression (e.g., Constantine et al., 2003; Cohen \& Wills, 1985; 
Coffman \& Gilligan, 2002), academic performance (e.g., Hinderlie \& Kenny, 2002; Murray et al., 2012; Wilks \& Spivey, 2010) and psychosocial-emotional adjustment to college (e.g., Burleson \& Mortenson, 2003; Chang, 2001; Felsten \& Wilcox, 1992; Halamandaris \& Power, 1999; Hinderlie \& Kenny, 2002; Lamothe et al., 1995; Mortenson, 2006; Rodriguez, Mira, Myers, Morris, \& Cardoza, 2003; Sanders \& DuBois, 1996; Winter \& Yaffe, 2000; Wodka \& Barakat, 2007).

Perceived social support has also been noted in the literature as a valuable intrapersonal coping strategy in college adaptation among traditionally under-represented student populations, such as minority students (e.g., Latino, Black, Asian, Asian-American, Chinese, Mexican-American; Muslim) (e.g., Burleson \& Mortenson, 2003; Chang, 2001; Constantine et al., 2003; Crockett et al., 2007; Hinderlie \& Kenny, 2002; Mallinckrodt, 1988; Mortenson, 2006; Rodriguez et al., 2003; Solberg \& Villarreal, 1997; Tao, Dong, Pratt, Hunsberger, \& Prancer, 2000; Yalcin, 2011; Zea et al.,1995). However, only a handful of studies have explored the predictive role of perceived social support in college adjustment outcomes among undergraduate students with disabilities. With few exceptions (Winterowd et al., 1998; Wodka \& Barakat, 2007), such research efforts have typically focused on establishing the presence of relevant stresses, instead of investigating the possible influence of perceived social support in psychosocial adjustment to college (e.g., Cosden \& McNamara, 1997; Sanders \& DuBois, 1996; Spekman, Goldberg, \& Herman, 1992). Moreover, generalizability of the findings are severely restricted in many of these 
studies, due to their relatively small sample sizes (e.g., Sanders \& DuBois, 1996; Wodka \& Barakat, 2007). The current study therefore, sought to contribute to the empirical literature by exploring relationships between perceived social support and adaptation-associated college adjustment outcomes in lower division students with disabilities.

Perceived social support as moderator. In addition to its direct, predictive relationship with adaptive postsecondary outcomes, perceived social support has also been portrayed as a moderator variable that protects an individual against the negative consequences of stress and, hence, its effect has been evident only at high levels of stress (e.g., Cobb, 1976; Cohen \& Hoberman, 1983; Crockett et al., 2007; Folkman \& Moskowitz, 2004; Ptacek \& Pierce, 2003; Sek, 1991). Perceived social support may serve as a moderator against stress by preventing a situation from being appraised as stressful, by providing a solution to a stressful problem, and/or by facilitating healthy, adaptive behavioral responses (Cohen \& Wills, 1985). Research supports the role of intrapersonal processes, such as perceived social support, moderating the adverse psychological impact of exposure to stressful life events related to college adjustment (e.g., Billings \& Moos, 1981; Cassel, 1976; Cohen \& Wills, 1985; Crockett et al., 2007; Folkman \& Moskowitz, 2004; Heller \& Swindle, 1983; Ptacek \& Pierce, 2003; Sek, 1991; Swift \& Wright, 2000). However, no studies were found in the literature that investigated perceived social support as a moderator in the relationship between negative life events (e.g., college stress; functional limitations) and 
adaptation-associated college adjustment outcomes among undergraduate students with disabilities. The current study addressed this void in the literature by exploring perceived social support as a potential moderating variable, based on specific hypothesized relations, in first-year and second-year undergraduate students with disabilities.

\section{Adaptation-Associated Outcomes: A Quality of Life Framework}

Too often, rehabilitation psychology (e.g., Antonak \& Livneh, 1995; Bishop, 2005; Dunn, 2005; Dunn \& Dougherty, 2005; Livneh, 2001; Livneh \& Antonak, 2005) and education (e.g., Dyson \& Renk, 2006; Mooney, Sherman, \& LoPresto, 1991; Zea, Belgrave, Townsend, Jarama, \& Banks, 1996) researchers have operationalized adaptation-associated outcome measures using univariate (i.e., measured by items that are summed to yield a single score, representing degree or level of adjustment) and negatively skewed (i.e., deficit-oriented, such as the absence or presence of anxiety or depression) approaches. For example, a study by Dyson and Renk (2006) explored coping and adaptation to university life among 74 first-year college students. These researchers operationalized college adjustment by using the Beck Depression Inventory-II (BDI-II; Beck, Steer, \& Brown, 1996) to assess symptoms of depression consistent with the depressive disorders listed in the Diagnostic and Statistical Manual of Mental Disorders, Fourth Edition-Text Revision (American Psychiatric Association, 2000). Level of depressive symptomatology was therefore selected as the criterion variable. Although psychological dysfunction has often been used as an indicator of 
adjustment, Diener and Larsen (1993) noted that successful adaptive outcomes should not simply reflect the absence of pathology. Such a perspective yields only a partial picture of adjustment, and is not able to register any positive aspects of individuals' lives (Folkman \& Greer, 2000; Folkman \& Moskowitz, 2000). A more holistic and balanced view of psychosocial adaptation has been identified in the field of positive psychology (Seligman, Steen, Park, \& Peterson, 2005). Positive psychologists recognize that the human experience post-CID has not exclusively been that of psychosocial distress or suffering, but does, indeed, offer the potential for growth, life satisfaction, and finding meaning in life. The measurement of adaptation-associated outcomes should, therefore, include positive indicators that can give researchers a broader and more complete picture of the disability experience. Such a perspective also promotes the pursuit of lifestyles and behaviors that are conducive to better mental health (Seligman et al., 2005).

One positive indicator of adaptation that has been increasingly advanced by researchers in the fields of positive psychology (e.g., Seligman et al., 2005; Dunn, 1996; 2005; Dunn \& Dougherty, 2005), rehabilitation psychology (e.g., Bishop, 2005; Bishop et al., 2009; Dunn, 1996, 2005; Dunn \& Dougherty, 2005) and education (e.g., Zea et al., 1996; Zullig, Huebner, Gilman, Patton, \& Murray, 2005; Zullig, Huebner, \& Pun, 2009) is the individual's appraisal of satisfaction in various life domains. In fact, there are a number of researchers who view life satisfaction as the crux of positive adjustment. Some researchers are interested in overall appraisals of 
general (i.e., global) life satisfaction, whereas others focus on satisfaction in specific life domains, such as family, education, recreation, and vocation (Diener, Suh, Lucas, \& Smith, 1999). Both general and specific conceptualizations have been referred to in the rehabilitation psychology and education literatures as subjective well-being, or “quality-of-life," (QoL) (e.g., Diener et al., 1999; Dunn, 2005; Zullig et al., 2005, 2009), relying on the "standards of the respondent to determine what is the good life" (Diener, 1984, p. 543).

QoL also offers an appraisal of human functioning that can be both objective (typically associated with socio-demographic data, such as employment, income, economic status) and subjective (i.e., self-appraisal in overall life satisfaction, or in various life domains) in nature. QoL, therefore, represents a framework of human functioning that can encompass a wide range of life domains, including global life satisfaction, well-being (e.g., Diener \& Seligman, 2004; Diener et al., 1999;

Frisch et al., 2005), as well as specific domains (e.g., health domain, educational domain, recreational domain, vocational domain) (Frisch, 2000; Livneh, 2001). In the current study, the broader QoL construct was conceptualized more specifically as an individual's subjective appraisal of college-related adaptation-associated outcomes. These outcomes included appraisals of life satisfaction, as well as the academic and psychosocial-emotional domains of college life.

\section{The QoL-PACID Model: An Introduction}

The construct of adaptation has been studied from the perspective of a number of 
disciplines (e.g., psychology, education, sociology, rehabilitation psychology) and from the viewpoint of a number of extant theories (Bishop et al., 2009; Livneh, 2001; Livneh $\&$ Martz, 2012). Although specific models differ with regard to their essential components, processes, dynamics, and temporal relationships among proposed factors, most share similar perspectives on the nature of human adaptation (Livneh \& Martz, 2012). In the current study, (un)successful adaptation to college among first-year and second-year undergraduate students with disabilities was conceived as subjective quality-of-life (QoL), measured by self-appraisals of: global life satisfaction, academic performance, and psychosocial-emotional adjustment to college. Because the current study considered disability-related characteristics as possibly influencing a normative transitional event (i.e., adjustment to college) that was specific to a population of undergraduate students with varying chronic illness and disability conditions, the model selected to investigate the relationships between selected variables (i.e., college stress, functional limitations, intrapersonal coping [engagement-type coping strategies; disengagement-type coping strategies; perceived social support]) and QoL adaptation-associated outcomes, came from the field of rehabilitation psychology. The specific model was based on a positivist, QoL framework, called the Quality-of-Life Psychosocial Adaptation to Chronic Illness and Disability (i.e., QoL-PACID) model. Because the model will be discussed more fully in Chapter II (i.e., Review of the Literature), it is only briefly highlighted in this section of the current study.

The Quality-of Life Psychosocial Adaptation to Chronic Illness and Disability 
(i.e., QoL-PACID) model identified by Livneh (2001) and colleagues (Bishop, 2005; Bishop et al., 2009; Livneh, 2001; Livneh \& Antonak, 1997, 2005) consists of three main components: antecedents, processes, and outcomes of adaptation.

The first component, antecedents, includes two interacting sets of variables: CID-triggering events (such as genetics, injury, and chronic illnesses), and contextual variables (existing biological, psychosocial, and environmental conditions).

The two sets of background variables (i.e., CID-triggering events and contextual variables) exert a substantial influence upon the next two sets of interacting factors, categorized under the "process" component. These include, first, unfolding psychosocial reactions to the onset of CID and, second, contextual influences that exist (and may also shift) during the period following CID onset. The experienced reactions are those commonly reported by people following the onset of CID, such as denial, anxiety, depression, and anger. The contextual influences refer to those dynamic and interacting forces that, directly or indirectly, affect the nature, valence, and progression of the adaptation process. Furthermore, these continuously evolving external and internal forces also refer to the intrapersonal, interpersonal, and environmental influences that undergo constant changes during the process of adaptation.

Unlike typical models of college adjustment found in the higher education literature (e.g., Bean, 1980, Russell \& Petrie, 1992; Tinto, 1987, 1993), the QoL-PACID model takes into consideration predictor-related components that have been considered relevant in the daily lives of individuals with disabilities. Specifically, 
it has been proposed that a host of interacting factors, including intrapersonal (i.e., those variables associated with personality [e.g., optimism, neuroticism] or psychological attributes [e.g., coping strategies, perceived social support, locus of control, self-esteem]), extrapersonal (i.e., those variables associated with external-environmental characteristics [e.g., reliability of social support network, supportiveness of the work environment]), and disability-related variables (i.e., those variables that are directly associated with a disabling condition [e.g., associated functional limitations, duration of condition, chronological age at the time of diagnosis]) can play a direct, as well as indirect role in the process of adaptation. These proposed factors (i.e., variables) can be explored as predictors, mediators, and/or moderators, in influencing (un)successful psychosocial adaptive outcomes.

The model also features adaptation-associated outcomes as the end product of the adaptation process, which also happens to equate with the concept of QoL (Bishop, 2005; Bishop et al., 2009; Livneh \& Martz, 2012), the overarching outcome criterion used in the current study to conceptualize and measure college adjustment.

\section{Intrapersonal Coping and Adjustment to College}

The only empirical study found in the literature that addressed the role of both coping and perceived social support in adjustment to college among undergraduate students with disabilities was conducted by Wodka and Barakat (2007). These researchers assessed psychological adjustment to college using the total symptoms scores of the Beck Anxiety Inventory (BAI) (Beck \& Steer, 1993), and the Beck 
Depression Inventory-II (BDI-II) (Beck et al., 1996). The perception of supportive social relations, as measured by the Social Support Questionnaire (SSQ; Sarason, Levine, Basham, \& Sarason, 1983), was linked to positive psychological adjustment (lower levels of anxiety, as measured by the BAI). Coping, measured by the 60 item COPE (Carver, Scheier, \& Weintraub, 1989), was also directly linked to psychological adjustment. Specifically, passive, disengagement-type coping (i.e., denial, mental and/or behavioral disengagement, inappropriate alcohol/drug use) was related to poorer psychological adjustment (i.e., higher levels of anxiety). Both intrapersonal variables investigated (i.e., disengagement-type coping strategies, perceived social support), thus played a significant role in adaptation to college among first-year and second-year students with disability. That study, however, (a) did not examine the potential moderating, stress-interacting role of intrapersonal variables (i.e., engagement-type coping; perceived social support) in linking the relation between stress and adaptation, (b) included only respondents with physical chronic illnesses, (c) did not explore the role of any disability-related variables, and (d) assessed adaptation-associated outcomes using negative, deficit-oriented outcome measures (i.e., examining the presence or absence of anxiety/depression), instead of measures reflecting a holistic, multidimensional and positive psychology approach (i.e., life satisfaction/well-being; specific college-related QoL domains).

\section{Purpose of the Study}

The purpose of the current study was to develop a better understanding about the 
adjustment of lower division undergraduate college students with disabilities. There was a particular need in developing insight regarding the role of engagement-oriented coping strategies and perceived social support (each) in promoting (un)successful adjustment to college among students with disabilities generally, and the moderating effects of such intrapersonal coping efforts on relationships between college stress (i.e., college-related characteristic), functionality (i.e., disability-related characteristic), and adjustment to college outcomes in first-year and second-year undergraduate students with disabilities, based on specific hypothesized relations. In addition, adjustment to college was examined using three adaptation-associated outcome indicators that reflected a positive and multidimensional QoL perspective. Namely, one global indicator of QoL (i.e., life satisfaction), and two college-specific indicators of QoL (i.e., academic performance; psychosocial-emotional adjustment to college).

An exploration of these variables may be helpful in determining why some undergraduate students with disabilities may feel overwhelmed, while others thrive, in response to the challenges and demands of college life. Moreover, an empirical investigation of specific intrapersonal coping resources (i.e., engagement-type coping; perceived social support) to reduce or ameliorate (i.e., moderating model) negative effects of possible stress (i.e., college stress; functionality) on adjustment offers a potentially viable approach for supporting students with disabilities during college. Findings may be especially helpful in guiding rehabilitation and education professionals in the selection of therapeutic interventions that will promote optimal college 
adjustment in lower division students with chronic illness and disability.

\section{Study Aims, Hypotheses \\ and Research Questions}

Three research aims, along with one sub-aim were included in the current study. The first aim of this investigation was to explore simple relationships between each of the study's four predictor variables (i.e.. college stress, functional limitations, coping [i.e., engagement-type; disengagement-type], and perceived social support), and three adaptation-associated criterion measures of (un)successful college adjustment (i.e., life satisfaction, academic performance, and psychosocial-emotional adjustment to college). The second, and primary aim of the study was to examine engagement-type coping and perceived social support (each) as possibly moderating the relationships between perceived negative life events (i.e., college stress; functionality) and three QoL-based adaptation-associated college adjustment outcomes (i.e., life satisfaction, academic performance, psychosocial-emotional adjustment to college). The third aim of the study was to explore several socio-demographic variables and their possible relationship to each of the study's three adaptation-associated criterion variables (i.e., life satisfaction, academic performance, psychosocial-emotional adjustment to college) in adjustment to college. The last aim, and sub-aim of this exploratory study was to investigate disengagement-type coping and it's possible contribution to the variance in adaptation-associated college outcomes.

First and second research aims. The first and second research aims of the current study were addressed by eight specific hypotheses. These included: (a) examining 
the relationship between college stress and adaptation, (b) examining the relationship between functionality and adaptation, (c) examining the relationship between engagement-type coping and adaptation, (d) examining the relationship between perceived social support and adaptation, (e) examining the role of engagement-type coping as a moderator in the relationship between college stress and adaptation, (f) examining the role of engagement-type coping as a moderator in the relationship between functionality and adaptation, (g) exploring the role of perceived social support as a moderator in the relationship between college stress and adaptation, and (h) exploring the role of perceived social support as a moderator in the relationship between functionality and adaptation. The following eight hypotheses were proposed:

1. There will be a negative relationship between college stress and adjustment to college, as measured by: (a) life satisfaction, (b) academic performance, and (c) psychosocial-emotional adjustment to college, in lower division students with disabilities.

2. There will be a negative relationship between functionality (more pronounced functional limitations) and adjustment to college, as measured by: (a) life satisfaction, (b) academic performance, and (c) psychosocial-emotional adjustment to college, in lower division students with disabilities.

3. There will be a positive relationship between engagement-type coping and adjustment to college, as measured by: (a) life satisfaction, (b) academic performance, and (c) psychosocial-emotional adjustment to college, in lower 
division students with disabilities.

4. There will be a positive relationship between social support and adjustment to college, as measured by: (a) life satisfaction, (b) academic performance, and psychosocial-emotional adjustment to college, in lower division students with disabilities.

5. Engagement-type coping will moderate the impact of college stress on adjustment to college, as measured by: (a) life satisfaction, (b) academic performance, and (c) psychosocial-emotional adjustment to college, in lower division students with disabilities.

6. Engagement-type coping will moderate the impact of functionality (more pronounced functional limitations) on adjustment to college, as measured by:

(a) life satisfaction, (b) academic performance, and (c) psychosocial-emotional adjustment to college, in lower division students with disabilities.

7. Perceived social support will moderate the impact of college stress on adjustment to college, as measured by: (a) life satisfaction, (b) academic performance, and psychosocial-emotional adjustment to college, in lower division students with disabilities.

8. Perceived social support will moderate the impact of functionality (more pronounced functional limitations) on adjustment to college, as measured by: (a) life satisfaction, (b) academic performance, and (c) psychosocial-emotional adjustment to college, in lower division students with disabilities. 
Third research aim. This study's third research aim was to investigate the role of several socio-demographic variables that may be associated with adjustment to college among lower division undergraduate students with disabilities. Namely, duration of disability, chronological age of respondent, gender, hours employed (per week), and disability group (i.e., physical; cognitive/neurocognitive; psychiatric) membership differences that may be associated with college adjustment (as measured by life satisfaction, academic performance, and psychosocial-emotional adjustment to college). To investigate socio-demographic factors that might have special relevance for understanding college adjustment, the following five exploratory research questions were posed:

1. To what extent is time since diagnosis of disability (i.e., duration of disability) related to college adjustment, as measured by: (a) life satisfaction, (b) academic performance, and (c) psychosocial-emotional adjustment to college, in lower division students with disability?

2. To what extent is age of the respondent related to college adjustment, as measured by: (a) life satisfaction, (b) academic performance, and (c) psychosocial-emotional adjustment to college, in lower division students with disability?

3. To what extent is gender related to college adjustment, as measured by: (a) life satisfaction, (b) academic performance, and (c) psychosocial-emotional adjustment to college, in lower division students with disability? 
4. To what extent is hours employed (per week) related to college adjustment, as measured by: (a) life satisfaction, (b) academic performance, and (c) psychosocial-emotional adjustment to college, in lower division students with disability?

5. Are there significant disability group membership differences (i.e., physical disability group, cognitive/neurocognitive disability group, psychiatric disability group) as related to the study's three criterion measures of college adjustment (i.e., life satisfaction, academic performance, psychosocial-emotional adjustment to college) in lower division students with disability?

Sub-aim of study. In addition to the five exploratory research questions, the current study also included one exploratory sub-aim. Specifically, the sub-aim explored the unique contribution of disengagement-type coping to college adjustment (as measured by the study's three adaptation-associated outcome measures [i.e., life satisfaction, academic performance, psychosocial-emotional adjustment to college]), after controlling for relevant socio-demographic variables. The following research question was posed:

1. To what extent does disengagement-type coping account for variance in adjustment to college, as measured by: (a) life satisfaction, (b) academic performance, and (c) psychosocial-emotional adjustment to college, in lower division students with disability? 


\section{Definitions of Key Terminology}

This section of the introductory chapter provides definitions to key terminology used throughout the study.

Adaptation: The term "adaptation" is used interchangeably with the term "adjustment," and represents the end-phase of the transition to college process.

Chronic illness and disability (CID): A physical, cognitive, or psychiatric condition that causes functional limitations that substantially limit one or more major life activities including mobility, communication (e.g., seeing, hearing, speaking), and learning.

Coping: A person's constantly changing cognitive and behavioral efforts to manage specific external and/or internal demands that are appraised as taxing or exceeding the resources of the person.

Functionality: A neutral term that pertains to the functional aspects of living with a disability. Defined descriptively as to what an individual can and cannot do in relation to the performance of everyday activities and life roles.

Functional limitations: Restrictions in the performance of everyday activities and life roles.

Perceived social support: The belief or perception that one is cared for and loved, esteemed and valued, and a member of a network of mutual support. The term "perceived social support" is also equated with the "esteem support" typology or function of social support. 
Quality-of-Life (QoL): The subjective appraisal of overall adjustment to college, comprised of one global indicator of well-being/satisfaction with life (i.e., life satisfaction), and two college-specific indicators representing academic and psychosocial-emotional domains of the college environment. Also referred to in this study as "subjective Quality-of-Life.”

Transitions: Periods of change, disequilibrium, and internal conflict about gains and losses that occur between periods of stability, balance, and relative quiescence. The end-phase or outcome of the transition process is called "adaptation" or "adjustment." 


\section{Chapter II}

\section{Review of the Literature}

The primary focus of Chapter II is the topic of adaptation. The chapter begins with a brief overview of college adjustment, particularly as it applies to undergraduate students with disabilities, and concepts relevant to the current study. The focus then shifts to the construct of adaptation, from its historical roots, to those theoretical approaches of adaptation to CID that have been considered the most influential conceptual models of psychosocial adaptation. A brief discussion of the theoretical and clinical limitations associated with these various approaches or frameworks is also included. It is then proposed that a quality-of-life based perspective on adaptation be used to encompass the salient features of these reviewed extant frameworks. Following this, a specific model of adaptation to chronic illness and disability (CID), termed the Quality of Life (QoL)-based Psychosocial Adaptation to Chronic Illness and Disability Model (QoL-PACID) (Bishop, 2005; Bishop, Smedema, \& Lee, 2009; Livneh, 2001; Moos \& Holahan, 2003, 2007; Moos \& Schaefer, 1993) is proposed as the guiding framework of this study. This model integrates concepts drawn from the QoL literature with existing models of adaptation from the rehabilitation psychology literature. The discussion of the QoL-PACID model includes its application for exploring the proposed predictors (i.e., college stress; functional limitations; engagement-oriented coping; disengagement-oriented coping; perceived social support] and intrapersonal moderator variables (i.e., engagement coping; perceived social support), and their hypothesized 
relationships to the study's three adaptation-associated outcome variables: life satisfaction, academic performance, and psychosocial-emotional adjustment to college.

\section{Overview: Students with Disabilities and Adjustment to College}

Attending a college or university for the first time marks an important major life transitional event in American society. Postsecondary entrance is often regarded as a very positive life experience, with great opportunities for personal growth, development, and achievement. However, as with other major life transitions, it can also be a challenging, demanding, as well as potentially stressful experience. (e.g., Appelhans \& Schmeck, 2002; Hickman, Bartholomae, \& McKenry, 2000; Misra \& McKean, 2000; Morosanu et al.,2010; Morrison \& O’Connor, 2004; Tobin \& Carson, 1994; Trockel, Barners, \& Egget, 2000).

Among individuals transitioning to undergraduate college life, a constellation of stressors is often experienced (e.g., Blimling \& Miltenberg, 1981; Frazier \& Schauben, 1994; Morosanu, Handley, \& O'Donovan, 2010; Mortenson, 2006; Russell \& Petrie, 1992), including those factors related to (a) academics (e.g., Appelhans \& Schmeck, 2002; Macan, et al., 1990; Misra, 2000; Misra \& Castillo, 2004; Russell \& Petrie, 1992; Trueman \& Hartley, 1996), (b) financial pressures (e.g., Frazier \& Schauben, 1994; Kim et al., 2012; Miech \& Shanahan, 2000; Nelson et al., 2008), and (c) social relationships (e.g., Beck, Taylor, \& Robbins, 2003; Lopez, Mauricio, Gormley, Simko, \& Berger, 2001; Morosanu et al., 2010).

Academic stressors. Academic-related stress may arise from challenging course 
loads, grade competition, and issues relating to time or task management (Macan, Shahani, Dipboye, \& Phillips, 1990; Trueman \& Hartley, 1996). There may also be difficulties in adjusting to a new learning environment that may present higher academic demands than accustomed to (Appelhans \& Schmeck, 2002; Blimling \& Miltenberger, 1984; Coffman \& Gilligan, 2002; Fram \& Bonvillian, 2001; Misra, 2000; Misra \& McKean, 2000; Misra \& Castilo, 2004; Russell \& Petrie, 1992; Van-Rooijens, 1996). Students may also doubt whether they will be able to meet the academic expectations of parents and/or friends, in addition to the expectations that they may have for themselves (e.g., Blimling \& Miltenberger, 1984; Kitzrow, 2003; Misra, 2000; Lecompte, 1986; Pancer, Hunsberger, Pratt, \& Alisat, 2000; Pierceall \& Keim, 2007; Pritchard \& Wilson, 2003; Tinto, 1993).

Financial stressors. In terms of financial pressures, there may be challenges in managing one's personal finances for the first time, or in having adequate funds to pay for tuition, food, housing, and/or recreation (Blimling \& Miltenberger, 1981; Miech \& Shanahan, 2000; Nelson et al., 2008; Ross et al., 1999). There may also be financial-related burdens due to student loan debt, or credit card debt (Kim et al., 2012; Nelson et al., 2008).

Social stressors. Postsecondary entrance may cause disruption of an individual's social support system, and/or level of perceived social support (e.g., Beck, Taylor, \& Robbins, 2003; D'Aurora \& Fimian, 1988; Hinderie \& Kenny, 2002; Lapsley, Rice, \& Shadid, 1989; Mortenson, 2006; Prillerman et al., 1989; Russell \& Petrie, 1992; Solberg 
\& Villarreal, 1997; Winter \& Yaffee, 2000; Zea, Jarama, \& Bianchi, 1995). For example, individuals undergoing this transition often lose access to the protective environment of family members and close friends (Beck et al., 2003). Stress may also arise due to the unfamiliarity of postsecondary life challenging an individual's need for acceptance and comfort (Blimling \& Miltenberg, 1981; Young, 2003). Challenges in developing and maintaining satisfying interpersonal relationships, may also potentially lead to increased levels of stress (e.g., Blimling \& Miltenberg, 1981; Hilsman \& Garber, 1995; Jones, 1993; Kitzrow, 2003; Pritchard \& Wilson, 2003; Solberg et al., 1998). Most undergraduate postsecondary students are beginning their transition from adolescence to adulthood. In fact, Arnett (2000) proposed the term emerging adulthood to describe the culturally constructed period of extended adolescence that occurs in industrialized countries when higher education (or some other form of preparation for adulthood) is undertaken. During their emerging adulthood, individuals are still forming an individual identity; a process that includes attaining autonomy from parents, forming a gender identity, and internalizing morality (Arnett, 2000; Newman \& Newman, 1995). Individuals in this life stage are also establishing themselves as productive members of society by integrating vocational goals, developing the capacity for intimate relationships, and accepting social responsibility (Falvo, 1999). When individuals feel pressured by these multiple developmental tasks, they can experience role-strain, role-overload, and role-ambiguity, which may result in intense feelings of stress (Goldman \& Wong, 1997; Humphrey \& McCarthy, 1998). 
Older college students, on the other hand, may have fears about entering the undergraduate postsecondary environment (Carson, Butcher, \& Mineka, 1998). More specifically, they may doubt their abilities to perform academic work as well as their younger peers attending college. In addition, older students may be, or believe themselves to be underprepared in their quests to make acceptable grades, maintain effective study habits, write acceptable papers, and study for examinations. These concerns may be coupled with the demands of working, juggling household chores, caring for family members, and struggling with limited income that has to be stretched to pay for college tuition. Although some older students may handle such demands with relative ease, others may find them too stressful or overwhelming to deal with, leading to college adjustment problems (e.g., poor GPA; alcohol/drug abuse; anxiety and/or depression), or even withdrawal from a college or university.

The typically unique and multiple college-related demands associated with the major life transition of attending college for the first time, may thus prove to be a crucial test of an individual's ability to cope and adapt to such an environment. The transition to college may be more challenging however, for those students concurrently dealing with other major life demands, such as individuals with chronic illness and disability (CID).

According to the rehabilitation psychology literature (Falvo, 1999; Horowitz, 1985; Livneh \& Wilson, 2003), individuals with CID normally face an increase in both the frequency and severity of stressful situations. Among the more commonly 
recognized disability-related factors that may interact to create increased stress on the lives of individuals with CID include: the degree or level of functional limitations, the prolonged course of medical or psychiatric treatment, the impact on family and friends, and the threat of economic stability (e.g., increased health costs; increased insurance costs; potential for reduced income). In particular, disability-related functional limitations have been cited as an important predictor of psychosocial adaptive outcomes among individuals with a wide variety of chronic illness and disability conditions (Livneh \& Wilson, 2003). Studies on adjustment to CID reveal that severe impediment with functional ability (i.e., as measured by increased limitations in the ability to perform usual daily tasks and life roles) is often associated with poorer psychosocial adjustment outcomes (e.g., depression, psychosocial distress, decreased sense of purpose in life (Dunn, 1996, Eide \& Roysamb, 2002; Ferington, 1986; Haden et al., 2007; Wineman, 1990, Wineman et al., 1994). However, no empirical studies have examined the role of disability-related functional limitations as a predictor of psychosocial adaptation to college among students with CID's.

The demands of living with a CID can also pose challenges that may be in direct conflict with emerging (i.e., age 18 to 25 years), or older (i.e., age 26 and older) adulthood (Falvo, 1999). For example, the limitations imposed by the CID, rather than the interests or abilities of the individual, may define occupational goals. Physical, cognitive/neurocognitive, or psychiatric limitations may also inhibit an individual's effort to establish new relationships, or to maintain relationships that have already been 
built. For individuals who have not yet fully gained independence, or left their family of origin by the onset of the CID, achieving independence may become more difficult. Barriers to gaining independence may include limitations imposed by the CID, or over-protectiveness by parents. For younger adults, protective reactions by family members may also hinder the individual's attainment of autonomy and individual identity. Consequently, undergraduate students who also experience CID may be faced with profound challenges related to their disability (i.e., disability-related demands), while also dealing concurrently with the unique challenges pertaining to college life (i.e., college-related demands).

Adaptation, or the individual's adaptive response to the experience of a major life transition (e.g., Schlossberg, 1981) or crisis-like situation (e.g., Bishop, 2005; Bishop et al., 2009; Livneh, 2001; Moos and Schaefer, 1984), is a complex, highly individual, and multidimensional process, influenced by a number of intervening variables. Rather than a stable state, adaptation is conceived as a dynamic process that evolves over time. Although determining when an individual has completed the process is nebulous, typically some version of productivity is used to assess the level of adaptation an individual has reached. In addition, definitions of adaptation vary. In the current study, adaptation to college among first-year and second-year undergraduate students with disabilities is conceived as: the decision to remain or persist in college, along with self-appraisals of life satisfaction and performing well in various academic/psychosocial-emotional college-related domains, while dealing with 
concurrent college- and disability-related factors.

\section{Frameworks of Adaptation: A Rehabilitation Perspective}

The framework selected to investigate hypothesized relations between variables (i.e., college stress, functional limitations, intrapersonal coping [engagement-type coping; disengagement-type coping; perceived social support]) and adaptation-associated college outcomes (i.e., life satisfaction, academic performance, psychosocial-emotional adjustment to college) in lower division students with disabilities, comes from a rehabilitation psychology perspective. The specific model is broadly based on a positivist perspective, and is conceived in terms of a QoL framework of psychosocial adaptation to chronic illness and disability (i.e., PACID), more conveniently identified in the current study as the QoL-PACID model.

The QoL-PACID model is the guiding framework for the current study's conceptualization and empirical exploration of adaptation and adaptation-associated outcomes, based on specific hypothesized relations. Although all models share certain perspectives on the nature of adaptation to human adversity, they do differ in their philosophical-theoretical underpinnings and the complexities of the proposed systems (Livneh \& Martz, 2012). The following is a review of those historical and contemporary frameworks of adaptation to chronic illness and disability (CID) considered most influential in rehabilitation psychology research and clinical practice, and some of the more salient limitations that have been associated with them. The specific frameworks include: (a) the medical model, (b) the stage/phase model, and (c) the ecological model. 


\section{The Medical Model}

Prominent early approaches to understanding the process of adaptation to CID were based on the medical model of disability (Bishop, 2005; Pledger, 2003; Smart, 2001; Tate \& Pledger, 2003). The medical perspective reduces disability to a problem of individual functioning resulting from a disease or disorder that requires professional intervention. The unit of analysis is the patient, and adaptive outcomes are understood and measured in terms of the individual's level of functional improvement. Services and policies designed from a medical perspective tend to be provider driven, and focus on eliminating, to the extent possible, the condition that is causing the disability. According to the tenets of this paradigm, individuals who cannot be cured by professional intervention are placed in a role of dependency (i.e., the sick role) which exempts them from performing expected societal roles (Pledger, 2003; Smart, 2001; Tate \& Pledger, 2003).

\section{Limitations of the Medical Model}

Over time, clinicians, theorists and researchers in the rehabilitation psychology field rejected the narrow perspective of the medical paradigm. This model was reductive to pathology, and presented disability as the result of a deficit in an individual (Pledger, 2003). It also implied that individuals with disabilities were helpless objects, instead of actors in control, where their fate was concerned (Shontz, 1982). In addition, the medical model lacked empirical support and clinical utility for researchers and practitioners, respectively (Smart, 2001). The onset of a CID invariably 
triggers a chain of psychological reactions in the affected individual. The medical approach, however, ignored the individual's personal response to the onset of a CID (i.e., psychosocial reactions) (Livneh \& Antonak, 1997; Shontz, 1965; Smart, 2001).

\section{Stage/Phase Theories}

Stage/phase theories have dominated the adaptation literature for over forty years (e.g., Brooks \& Matson, 1982; Drudge, Rosen, Peyser, \& Pieniadz, 1986; Frank, Van Valin, \& Elliott, 1987; Gottesman \& Lewis, 1982; Lipowski, 1970; Shontz, 1965, 1975), with their development largely based on concepts borrowed from the mental health and grief and loss areas of psychology (Bowlby, 1973, 1980; Horowitz, 1985; Kubler-Ross, 1969; Parkes, 1975; Trieschmann, 1988; Wortman \& Silver, 1989). The majority of posited stage/phase models conceptualize adaptation to CID as comprised of an evolving, dynamic and progressive process, consisting of individually experienced psychosocial reactions (i.e., sets of experiences and reactions described as stages or phases), unfolding in a stable sequence, until the individual gradually achieves an optimal outcome that reflects psychological, social, and behavioral equilibrium (Livneh, 2001; Livneh \& Antonak, 1997; Shontz, 1965, 1975). Adjustment, on the other hand, refers more specifically to the clinically and phenomenologically hypothesized final stage/phase of the unfolding process of psychosocial adaptation (Livneh \& Antonak, 1997). The distinction between stages and phases is such that stages denotes discrete, categorically exclusive psychosocial reactions, whereas phases refers to psychosocial reactions that partially overlap or blend into one another (Livneh \& Antonak, 1997). 
Although there have been a number of different stage/phase models posited, the earlier models in particular, were unidimensional, and emphasized the linearity of the adaptation to CID process (e.g., Cohn, 1961; Falek \& Britton, 1974; Shontz, 1965). These models contained the similar premise of a generally predictable progression of stages or phases. The psychological experiences (i.e., reactions) were viewed as largely internally driven. Shock, anxiety, denial, depression, anger, acceptance, and finally some form of reorganization or final adjustment, are some of the reaction stages/phases postulated by these theorists concerning psychosocial adaptation to various CID's (e.g., Antonak \& Livneh, 1992; Devins \& Seland, 1987; Livneh, 1986, Livneh \& Antonak, 1997; Russell, 1981; Shontz, 1965; Weller \& Miller, 1977). In addition, the psychosocial reactions are essentially considered by their authors to be temporally ordered and hierarchical. That is, the order of emergence of these reactions is fixed, and each stage essential to achieve overall adaptation. For example, reaching the final stage (i.e., reorganization or adjustment) is conditional upon successful navigation of earlier stages such as denial or anger (Livneh \& Antonak, 2005).

An example of an earlier model of stage theory was proposed by Franklin Shontz (1965). His temporal model (1965) of response to disability contained five stages of psychosocial adaptation: shock, realization, defensive retreat, acknowledgement, and adjustment. The shock stage is designated as the initial stage of adaptation in which the individual experiences blunted emotional responses and shows minimal feelings and reactions. During the next stage, the stage of realization, Shontz proposed that 
individuals begin to approach and recognize the implications of their condition. As they come to acknowledge the reality and seriousness of their situation they react with anxiety, fear, depression, or anger. As a way of coping with the stress of this realization, the person moves into the next stage of adaptation, defensive retreat. In this stage, the individual denies the existence of the disability, or minimizes its seriousness as a means to combat fear and anxiety. When the reality of the situation becomes more apparent, and as individuals begin to find mechanisms to cope with anxiety, they move into the next stage of Shontz's model, called acknowledgement. During this fourth stage of adaptation, the individual reaches an understanding of the nature of the CID and its accompanying limitations. The final stage of this model is adjustment. During this stage, individuals have psychologically worked through their reactions to the CID and realistically accept their limitations. Individuals begin to plan for the future and focus on their abilities to reach their maximum potential.

Expanding on his earlier contribution to the literature, Shontz (1975) shifted his models' focus on the individual solely (i.e., personal reactions to crisis), to also reflect the impact of environmental influences in psychosocial adaptation to CID. Specifically, Shontz described successful adaptation to the response of disability as necessitated by two separate, evolving, and dynamic processes. Both processes involved a mutual accommodation of the individual's subjective experiences and external environment. On the one hand, internal perceptions led to actions that maximized an individual's available environmental opportunities. On the other hand, the environment must be 
adapted to facilitate an individual's efficacious behaviors. The better the fit between the psychological framework and the external reality, the better the degree of psychosocial adaptation. Adaptation is consequently perceived by Shontz as a function of the congruence between the subjective world of the individual and their external environment (i.e., person-environment congruence). Recognition of the role of external environmental influences in psychosocial adaptation to the onset of a CID represented an important conceptual advancement in the early literature regarding stage/phase models.

\section{Limitations of Stage/Phase Models}

An examination of the literature reveals limited consensus among researchers, theorists, and clinicians, regarding the premise of stage/phase theory (Kendall \& Buys, 1998; Livneh \& Antonak, 1997; Parker, Schaller, \& Hansmann, 2003; Trieschmann, 1988). Based on the available clinical and empirical evidence, Livneh and Antonak (1997) acknowledge that although a number of well-defined stages/phases have been described, the process is not always hierarchical or temporally ordered. That is, some individuals may skip stages/phases or revert to earlier stages/phases. Moreover, the concept of a final stage/phase of adaptation has been rejected as unrealistic for all individuals to achieve (Kendall \& Buys, 1998; Livneh \& Antonak, 1997; Parker et al., 2003).

Despite professional disagreements regarding the temporal and dynamic nature of psychosocial adaptation to CID, Livneh (2001) notes that most researchers and 
clinicians would probably acknowledge the existence of a series, or progression, of individually experienced reactions (also referred to as stages, phases, experiences, and responses). There is also clinical evidence supporting the classification of psychosocial reactions into short-term reactions (i.e., those immediately following the onset of a CID, such as shock and anxiety); intermediate reactions (e.g., grief, depression, hostility); and long-term reactions (i.e., those distally removed from onset of CID, such as passive and active acceptance, environmental mastery, behavioral adaptation, affective equilibrium, and integration). Moreover, the wide spectrum of reactions can range from adaptive (e.g., acceptance of CID, integration of CID into a newly and positively reconstructed self) to maladaptive (e.g., dependency, feelings of helplessness and hopelessness, denial of condition). There is also an implied assumption that these reactions are mostly internally originated (i.e., psychodynamic in nature), but can be amenable to environmental influences.

Traditional stage/phase models typically explain only one domain of the adaptation process, however (i.e., experienced reactions to the onset of a CID). They also provide little information about contextual variables (e.g., those associated with personality or psychological attributes; those associated with external environmental characteristics) that may directly, or indirectly, influence the process of adaptation. Moreover, it is likely that these contextual influences will provide the key to maximizing the effectiveness of counseling. Given these limitations, it has been suggested by numerous rehabilitation 
psychology experts that other frameworks or models be used to more accurately capture the dynamic and complex process of adaptation to CID (Bishop, 2005; Bishop et al., 2009; Livneh, 2001; Moos \& Holahan, 2003, 2007; Moos \& Schaefer, 1984, 1986, 1993; Parker et al., 2003).

\section{Ecological Models}

A more recent conceptualization of the process of psychosocial adaptation (e.g., to life crises, major life transitions, CID) represents a more structurally complex and comprehensive approach, in which focus is placed on the interaction between the individual and the external environment (e.g., Bishop, 2005; Bishop et al., 2009; Holahan, Moos, \& Schaefer, 1996; Livneh, 2001; Livneh \& Antonak, 1997; Livneh \& Parker, 2005; Moos, 1977; Moos \& Holahan, 2007; Moos \& Schaefer, 1984; 1993; Schlossberg, 1981; Scofield, Pape, McCracken, \& Maki, 1980; Trieschmann, 1988). Known as the ecological model, this paradigm provides a distinctive framework by which the transactions between individuals and their environments, and the impacts of these transactions on human functioning, can be understood and measured.

One of the most prominent and influential among the early ecological models was that of Nancy Schlossberg's (1981). In her ecological model of psychosocial adaptation to life transitions, Schlossberg stated that transitions occur if an event or nonevent results in a change in one's view about self or the world. Schlossberg suggested that adaptation to life transitions depends upon: the balance of the individual's available

personal, social, and environmental resources and deficits; and the differences between 
the pre-transition and post-transition environments. Moreover, her model identified three separate sets of factors that mediated psychosocial adaptation to transition: (a) characteristics inherent in the particular event or transition, (b) characteristics inherent in the pre-transition and post-transition contexts or environments, and (c) characteristics of the transitioning individual.

Characteristics inherent in the particular event or transition. Included here are variables such as the change (gain or loss) in life roles, the effect experienced by the change, the source of change (internal or external), the timing of the life events (on-time or off-time), the onset of the change (gradual or sudden), and the duration of the anticipated change (permanent, temporary, or uncertain).

Characteristics inherent in the pre-transition and post-transition contexts or environments. Included in this category are variables such as internal support systems (e.g., family unit, peer network), institutional supports (e.g., occupational organizations, religious institutions), and physical settings (e.g., climate, living arrangements, worksite).

Characteristics of the transitioning individual. Included in this set of factors are those socio-demographic (e.g., age, gender, ethnicity, socioeconomic status) and personality variables (e.g., psychosocial competence, attitudes, value and belief system, prior experience with transitions) that are inherent in the person himself or herself.

Schlossberg's (1981) model for analyzing human adaptation to life transition is often regarded as a milestone in the conceptualization of human adaptation to both 
anticipated (normative) and traumatic, crisis-like (non-normative) life situations. Ecological models adopting similar concepts to Schlossberg's have been proposed in the rehabilitation psychology literature. These models are specific to psychosocial adaptation to CID, and have typically been conceptualized and structured as either (a) linear-like (e.g., Holahan et al., 1996; Livneh \& Antonak, 1997; Moos, 1977; Moos \& Holahan, 2007; Moos \& Schaefer, 1984; Trieschmann, 1988), or (b) interactive-iterative (e.g., Livneh, 2001).

Linear-like models. These ecological models are typically integrative, and conceptualize the experience of psychosocial adaptation following a traumatic event, such as the onset of a CID, as essentially a linear process (Livneh \& Martz, 2012). Linear-like ecological models are more complex than the stage/phase models described in the previous section, as they implicate other determining factors in understanding variations in the process of psychosocial adaptation (e.g., Livneh, 2001; Livneh \& Antonak, 1997; Livneh \& Martz, 2012; Moos \& Holahan, 2007). These determining factors include: (a) CID-related characteristics (e.g., type, severity, and duration of condition), (b) socio-demographic characteristics (e.g., age, gender, religion), (c) personality and behavioral attributes (e.g., coping style, self-concept), and (d) external environmental influences (e.g., attitudinal barriers [i.e., social and cultural environment], architectural barriers [i.e., physical environment]). As noted by Livneh and Martz (2012), the role of these other determining factors has been typically viewed in literature as either (a) interactive or (b) mediating. When interactive processes are 
involved, the process of psychosocial adaptation follows different trajectories at different levels of the operating factor. For example, the severity of functional limitations (i.e., predictor variable) will have less influence on psychosocial adaptation to disability (i.e., outcome variable) when an engagement-oriented strategy (i.e., problem-focusing), rather than a disengagement-oriented strategy (i.e., avoidance) of coping (moderator variable) is adopted. With mediating processes, the implicated factor (for instance, specific adopted coping strategy) is seen as indirectly caused or influenced by an earlier variable (such as severity of functional limitations) and, in return, directly influences the outcome (such as psychosocial adaptation to CID). An example of a linear-like, ecological model comes from the work of Moos (1977) and his colleagues (e.g.,Billings \& Moos, 1984; Holahan et al., 1996; Moos \& Holahan, 2007; Moos \& Schaefer, 1984, 1986, 1993). These researchers proposed an integrative conceptual framework, whereby the psychosocial outcome of a life crises (i.e., CID) is first determined by three sets of components or factors (referred to as panels by Moos and his colleagues): (a) personal resources (Panel I), including intellectual ability, ego strength, cognitive and emotional maturity, self-confidence, belief system, and prior experience with illness and disability; (b) health-related factors (Panel II), including the rapidity of onset and progression of a condition, the severity of illness; and the (c) social and physical context (Panel III), such as family, friends, caregivers, as well as physical features of the home and workplace.

These three panels of the first model's component influence in concert the second 
component, which is made up of three linearly related panels: (a) cognitive appraisal (Panel IV), (b) adaptive tasks (Panel V), and (c) coping skills (Panel VI).

Cognitive appraisal. Cognitive appraisal acts as a kind of filtering device for the previous three panels and, in turn, influences the next two panels- adaptive tasks (Panel V) and coping responses (Panel VI). The cognitive appraisal panel, reminiscent of Lazarus and Folkman's (1984) conception of primary coping, refers to the perceived meaning that the person associates with the existence of CID. It also includes those perceptions regarding the condition's controllability, predictability, and changeability. Cognitive appraisals precede and partially determine the adaptive tasks (Panel V) that the person adopts to manage the CID.

Adaptive tasks. Adaptive tasks (Panel V) include a wide range of CID-generated tasks, such as those that focus on the management of symptomatology and treatment procedures, dealing with health-care providers, maintaining an emotional balance and positive self-image, sustaining relationships among family members and friends, and preparing for an uncertain future.

Coping skills. The third and final panel of the second component is that of coping skills. In the most recent version of their crisis and coping model, Moos and Holahan (2007) describe eight categories of coping classified along a 2 X 2 grid (approach vs. avoidance x cognitive vs. behavioral). Examples of these coping modes include logical analysis (cognitive/approach), seeking support (behavioral/approach), avoidance-denial (cognitive/avoidance), and emotional venting (behavioral/avoidance). 
The third and final component of the Moos and Holahan (2007) model consists of a single panel: well-being and health-related outcomes. This panel addresses the end product of the interactions among and progression of the earlier six panels. Although well-being and health-related outcomes are viewed as the final component of the model, the authors maintain that "in a mutual feedback cycle, health-related outcomes may alter the preceding sets of factors and consequently change longer-term health outcomes" (Moos \& Holahan, 2007, p. 109).

The Moos and Holahan (2007) model has achieved considerable empirical support. For example, in a longitudinal study of women with early-stage breast cancer, avoidance coping mediated the link between a partner's unsupportive behavior and subsequent emotional distress (Manne, Ostroff, Winkel, Grana, \& Fox, 2005). Consistent with the proposed model by Moos and colleagues, negative aspects of a relationship enhanced maladaptive coping efforts and, in turn, promoted poorer psychological adjustment to a diagnosis of breast cancer.

The Moos and Holahan (2007) model of coping with life crises has undergone several changes and refinements since its inception. According to Livneh and Martz (2012, p. 51) "it combines a rehabilitation-derived approach of grounded theory with accumulated empirical findings to the understanding of psychosocial adjustment to crisis and CID." One of its strengths, therefore, is its continuous growth from extensive review of earlier theoretical analyses and empirical research findings. The model is also clinically useful and offers rehabilitation psychologists fertile ground for working with 
diverse populations of people with CID.

Interactive-iterative models. Interactive-iterative ecological models of psychosocial adaptation to CID typically maintain that there is a reciprocal, iterative process of adaptation that involves both the individual and the environment (Livneh \& Parker, 2005). These models can be sequentially structured, and propose that the unfolding, dynamic, temporal process of psychosocial adaptation (i.e., experienced reactions following the onset of CID) proceeds in a complex manner that incorporates two distinct classes of interacting variables; namely, those internally (i.e., intra-individual variables), and those externally (i.e., environmental variables) associated with the individual. The intra-individual variables are those associated with both physical aspects (e.g., type and severity of CID, associated functional limitations) and psychological aspects (e.g., self-concept, self-esteem, locus of control, coping strategies) of the individual. These variables interact with existing environmental conditions that include those physical, social, political, cultural, spiritual, religious, recreational, and vocational elements associated with the individual. Psychosocial adaptive outcomes result from a continuous, iterative interaction between the individual and his or her environment.

The Quality of Life- (QoL) based, Psychosocial Adaptation to Chronic Illness and Disability (PACID), or QoL-PACID model used in the present study can be loosely considered an outgrowth of these ecological models. 


\section{Limitations of Ecological Models: The Process of Adaptation}

The ecological framework proposes that a host of variables are necessary to account for the complexity and variety of psychosocial responses to, and functioning after, the onset of a CID. It is argued by some rehabilitation psychology clinicians, researchers, and theorists, however, that contemporary models may not necessarily require many variables to explain complex behavior (Parker et al., 2003). Moreover, psychosocial behavior during adjustment to CID may not necessarily proceed in a stepwise, linear, or linear-like fashion (Parker et al., 2003; Trieschmann, 1988). In her rejection of a structured process regarding adaptation to spinal cord injury (SCI), Trieschmann (1988) noted:

... you can never understand a system by fragmentation and analysis alone. Unless you put the parts back into the context of the system, there is no understanding, no true knowledge, only collection of facts without coherence or unity. (Trieschmann, 1988, p. 49).

Trieschmann (1988) however, did acknowledged the importance of the intersection of the person and his or her environment, a concept on which the quality-of-life (QoL) framework of adaptation to CID is built upon.

There are also those rehabilitation psychologists who reject the linearity of the process of adaptation, in favor of a cyclical or recurrent model (i.e., pendular model). Although still in its infancy, such a model emphasizes the fully or partially circular or repetitive nature of most reactions to CID (Parker et al., 2003). Hence, adaptation is not a single, linear, or linear-like event, but rather a repeated series of experiences as new 
loses are encountered and assimilated (Livneh \& Martz, 2012; Parker et al., 2003).

\section{Limitations of Ecological Models: Outcomes of Adaptation}

Although assessment tools have been developed for the purpose of measuring the adaptive outcomes of a CID, those most commonly used in rehabilitation psychology research have been criticized for their (a) unidimensional nature, and (b) negatively skewed perspective (Bishop, 2005; Livneh, 2001; Smart, 2001; Wright \& Kirby, 1999).

Unidimensional nature. Extant outcome measures have frequently operationalized adaptation to CID as a unidimensional construct (Bishop, 2005), measured as, for example: (a) pathological dimensions of personality (typically the presence or absence of depression or anxiety); (b) physical or behavioral complaints; (c) changes in productivity or reduction in performance; or (d) degree of disability acceptance.

Negatively skewed perspective. Another criticism of extant measures regarding the impact of a CID is their tendency for a negatively skewed perspective (Bishop, 2005; Bishop et al., 2009; Smart, 2001; Wright \& Kirby, 1999). Although many individuals report psychologically positive aspects of living with a disability (e.g., Dunn, 1996, 2005; Dunn \& Dougherty, 2005; Folkman \& Greer, 2000), unidimensional measures of adaptation are typically deficit-orientated, based on the medical model of human functioning that focuses primarily on the presence or absence of pathology.

The unidimensional and deficit-oriented approach to defining and measuring adaptive outcomes fails to encompass the complex, holistic, and multidimensional nature of the individual's experience. Human functioning should therefore be perceived 
from a wide range of perspectives, including behavioral performance, psychological, vocational, and social-interpersonal (Bishop et al., 2009, Livneh, 2001; Smart, 2001).

\section{Theoretical Consensus}

Although there remain ongoing debates concerning the structure, process, and appropriate conceptualization of the outcomes (Bishop, 2005; Livneh \& Antonak, 1997), regarding psychosocial adaptation to disability and related crisis-like or stressful conditions, a review of the extant perspectives reveals a consensus on several points. First, irrespective of their structural components (e.g., linear, linear-like, pendular), and at its most fundamental level, adaptation is conceived as a dynamic, unfolding and temporal process (i.e., experienced psychological reactions, such as anxiety, denial, depression). Furthermore, initiation of this process is in response to the psychological, social, and functional changes that occur with the onset of a stressful event, crisis, or crises-like situation. Second, research implicates significant variation in terms of the individual's response to these changes (i.e., the adaptation process) (Kendall \& Buys, 1998). This variation can be attributed to the subjective and phenomenological nature of the individual's response to a complex interaction of contextual factors inherent in the disability itself (e.g., associated functional limitations, course of condition, severity of condition), the person's socio-demographic characteristics (e.g., age, gender, ethnicity, level of education, marital status), the person's personality (e.g., optimism, neuroticism) or psychological attributes (e.g., coping strategies, perception of supportive social relations; self-esteem) and the external environment (e.g., network of social support, 
institutional support, technological support) (Bishop, 2005; Bishop et al., 2009; Livneh, 2001; Livneh \& Antonak, 1997; Shontz, 1976, Wright, 1983). Furthermore, this process has been characterized in terms of movement towards some variously described adaptive outcomes, involving a wide scope of indicators that include both subjective (e.g., life satisfaction; perceptions of psychological well-being) and objective (e.g., health, vocational, educational, recreational) facets or domains of an individual's life (Bishop, 2005; Bishop et al., 2009; Livneh \& Antonak, 2005; Livneh \& Martz, 2012).

Given the multidimensional and highly subjective nature of the adaptation to CID process, several researchers and theorists in the field of rehabilitation (i.e., Bishop, 2005; Livneh, 2001; Moos \& Schaefer, 1993) have proposed that a multidimensional model of QoL can be an appropriate framework for understanding and assessing the personal impact of a CID, and the process by which individuals respond to this impact. Such a model is sufficiently broad to capture the range of impact across life domains, and is also able to portray the individual's subjective experiences with CID-related changes within those domains (Bishop, 2005). Moreover, this model represents a conceptual framework in which normative stressful life transitions involving individuals with a CID can be investigated.

My overarching objective in this study is, accordingly, to apply a Quality of Life (QoL)-based model of psychosocial adaptation to chronic illness and disability (PACID), to first-year and second-year undergraduate students with CID adapting to 
college. Furthermore, because of the large number of variables that can be included in this model, the current study will examine a trimmed-down model of those variables commonly implicated in the literature as influencing the process and outcomes of psychosocial adaptation, as applied to a postsecondary context (i.e., adaptation to college). The following section presents the Qol-PACID model.

\section{Quality-of Life-Based Model of Adaptation}

The development of the Quality of Life (QoL)-based model of psychosocial adaptation was influenced by several conceptual perspectives that have been proposed over the past forty-five years to address the structure and process of psychosocial adaptation to chronic illnesses and disabilities and related crisis-like or stressful conditions (e.g., Holahan et al., 1996; Livneh, 2001; Livneh \& Antonak, 1997; Moos, 1977; Moos \& Shaefer, 1984, 1986; Schlossberg, 1981; Shontz, 1965, 1975). Based on a synthesis of the reviewed literature, these extant perspectives or models implicate three distinct classes of interacting variables in influencing the nature, structure, and outcomes of the adaptation process; specifically (a) antecedent variables, (b) process-linked variables, and (c) adaptation-associated outcome variables.

Antecedent variables. Antecedent variables are those directly or indirectly linked to the triggering of the disabling condition (e.g., causes of CID, such as genetic disposition, birth trauma, accidents and injuries, diseases and illnesses, and conditions associated with the aging process), as well as those contextual conditions present at the time of CID onset. Contextual variables present at the time of CID onset can be 
situational and/or event-specific. They include all those biological/biographical (e.g., current health status, type and source of onset [i.e., gradual vs. sudden, internal vs. external], level of physical maturity, chronological age, gender, religion, ethnicity), psychosocial (e.g., personal and social identities; cognitive, emotional, and moral developmental phases; family and marital developmental phase; degree of resultant life threat), and environmental (existing external conditions such as physical, economic, social, and attitudinal conditions) variables that provide the backdrop to better understanding the impact of the CID on present and future psychosocial adaptation. It should be noted that a certain degree of overlap may exist among these variables. The two sets of background variables (i.e., CID-triggering events; contextual variables) exert considerable influence upon the second component of the model: process-linked variables.

Process-linked variables. The "process-of-adaptation" component of the QoL-PACID model is theoretically conceived as more dynamic and interactive than its "antecedent" component (i.e., triggering events and contextual variables associated with the occurrence of the disabling condition). Process-linked variables account for the temporal and dynamic nature of psychosocial adaptation, and refer more specifically to experiences that emanate from and follow the condition onset. Two broad domains that have been traditionally discussed within this context include: (a) psychosocial reactions (e.g., shock, anxiety, denial, depression), and (b) contextual influences that interact with life experiences after disability onset. 
Psychosocial reaction to CID. The model acknowledges that the onset of CID inevitable triggers certain subjectively experienced reactions in the affected individual. Although psychosocial reactions to CID vary, they often follow a certain clinical-phenomenological course. Psychosocial reactions typically described in the literature include anxiety, denial, depression, anger, acceptance or acknowledgement, and reintegration or adjustment (e.g., Dunn, 1996; Falvo, 1999; Shontz, 1975). Because psychosocial reactions are mostly internally originated (psychodynamic in nature), they may be modified by external events or direct interventions (e.g., counseling, environmental changes).

Contextual variables. The "process-of-adaptation" contextual influences refer to those dynamic and interacting forces that, directly or indirectly, affect the nature, valence, and progression of the adaptation process. These continuously evolving internal (e.g., the CID itself; personality [e.g., optimism, extravertism]; psychological [coping strategies employed; perceived social support; locus-of-control, self-efficacy]) and external (e.g., environmental; community-related) forces are commonly associated with personal, interpersonal, and environmental influences that undergo constant (ranging from subtle to more substantial) changes during the process of adaptation. These forces commonly stem from changes in functional capacities (i.e., functionality), course of the CID, the individual's self-concept, perceived control, sense of coherence, coping modalities, available social support, as well as encountered architectural and/or learning barriers. 
Adaptation-associated variables. The PACID-QoL model takes into consideration that the human experience post-CID is not necessarily negative, but can include such outcomes as happiness, life satisfaction, and well-being (Bishop, 2005; Bishop et al., 2009; Seligman, 2005), that can also be conceptualized as quality-of-life (QoL). QoL is a holistic, multidimensional concept that is depicted in the PACID-QOL model as the overarching outcome criterion of adaptation.

Researchers in the fields of rehabilitation psychology (e.g., Bishop, 2005; Bishop et al., 2009) and education (e.g., Zullig et al., 2005; Zullig et al., 2009) have commonly depicted QoL as consisting of three broad life domains: intrapersonal functioning (subjective well-being, life satisfaction, perceived health), interpersonal functioning (satisfaction with family life, peer relations, and social activities), and extrapersonal functioning (performance of educational/vocational activities and/or recreational pursuits, living arrangements). Adaptation to CID therefore, is determined by individuals' QoL across these global or specific life domains.

\section{Adapted Model}

Because the QoL-PACID model is inherently complex, and relies on a large number of interactive components, its proponents (Livneh, 2001; Livneh \& Martz, 2012) recommend a segmented approach to examining its various components and the proposed interactions among them. The current study therefore, examines a trimmeddown model of those variables commonly implicated in the theoretical and empirical literature as influencing the process and outcomes of psychosocial adaptation, as 
applied to a postsecondary context (i.e., adaptation-associated college adjustment). These variables include: college stress, functionality (increased restrictions in the ability to perform everyday activities and life roles), coping (engagement-type coping; disengagement-type coping [i.e., intrapersonal coping dimensions]); and perceived social support (i.e., intrapersonal coping dimension). Adaptation-associated college adjustment outcomes reflect a QoL perspective on adaptation, and include: life satisfaction, academic performance, and psychosocial-emotional adjustment to college.

Three research aims, along with one sub-aim include: (a) testing relationships between the study's predictor variables (i.e., college stress, functional limitations, engagement-type coping, and perceived social) and three adaptation to college outcome variables (i.e., life satisfaction, academic performance, psychosocial-emotional adjustment to college, (b) examining the moderating (interactive) role of engagement coping and perceived social support (each) in the relation between predictors (i.e., college-related predictor [college stress]; disability-related predictor [functional limitations]) and the study's three QoL adaptation-associated outcome variables (i.e., life satisfaction, academic adjustment, psychosocial-emotional adjustment to college); (c) exploring relationships between several socio-demographic variables (disability-related [duration of disability], person-related [respondents chronological age, gender] vocation-related [hours employed per week]) and the study's three outcome measures of college adaptation; and, (d) exploring whether disengagement coping contributes to the variance in adaptation to college (as measured by life 
satisfaction, academic performance, and psychosocial-emotional adjustment to college), beyond the contribution of relevant socio-demographic factors.

\section{Hypothesized Relationships}

This section highlights the adapted, trimmed-down QoL-PACID model which includes the proposed hypothesized relationships among the study's selected predictor (i.e., college stress, functionality, engagement coping, perceived social support) and moderator (i.e., engagement coping; perceived social support) variables, and QoL adaptation-associated indicators of college adjustment (i.e., life satisfaction, academic performance, and psychosocial-emotional adjustment to college). The discussion includes a brief review of some of the more salient empirical research findings associated with each hypothesized relation.

College stress and adaptation. Conceptualized as a process-linked variable in the adapted model, college stress is hypothesized to be significantly and negatively related to psychosocial adaptation to college among first-year and second-year undergraduate students with disabilities.

College stress has been studied extensively as an important variable influencing (un)successful adjustment to college (e.g., Baker \& Schultz, 1992; Baker, 2003; Dyson \& Renk, 2006; Edwards et al., 2001; Gall, Evans, \& Bellerose, 2000; Garret, 2001; Hammer, Grigsby, \& Woods, 1998; Kariv \& Heiman, 2005; Mallinckrodt, 1988; Misra \& McKean, 2000; Murray et al., 2012; Nelson et al., 2008; Saber et al., 2012; Wan, 1992; Zajacova, Lynch, \& Espenshade., 2005), and has been found to be directly related 
to theoretically relevant outcome criteria, such as life satisfaction (e.g., Coffman \& Gilligan, 2002; DeMakis \& McAdams, 1994; Saber et al., 2012; Solberg, Hale, Villarreal, \& Kavanagh, 1993; Yalcin, 2011), academic performance (Macan, Shahani, Dipboye, \& Phillips, 1990; Misra \& McKean, 2000; Morosanu et al., 2010; Pritchard \& Wilson, 2003; Struthers et al., 2000; Trueman \& Hartley, 1996), and psychosocial-emotional adjustment to college (Adams \& Proctor, 2010; Compas, Wagner, Slavin, \& Vannatta, 1986; Felsten \& Wilcox, 1992; Sanders \& DuBois, 1996). Functionality and adaptation. The second process-linked variable represented in the adapted model, functionality is a disability-related concept that is hypothesized to be significantly and negatively related to psychosocial adaptation to college among first-year and second-year undergraduate students with disabilities.

Functional aspects of CID have been described in the rehabilitation psychology and disability studies literature in terms of what an individual can and cannot do (e.g., Eide \& Roysamb, 2002; Heinemann, 2000; Heinemann \& Mallinson, 2010; Livneh \& Wilson, 2003; Wineman, 1990; Wineman et al., 1994), and are typically assessed by degree of functional limitations observed or reported. More specifically, Livneh and Wilson (2003) refer to functionality as "the inherent ability to perform various tasks, or to the behavioral component of the condition" (p. 195).

Functionality has been empirically documented in the rehabilitation psychology literature to be a predictor of psychosocial adaptation to CID (e.g., Eide \& Roysamb, 2002; Ferington, 1986; Kennedy, Lowe, Grey, \& Short, 1995; Lavigne \& 
Faier-Routman, 1992, 1993; Wineman, 1990). For example, in a study by Ferington (1986) involving persons with spinal cord injuries (SCIs), it was reported that functionality (more pronounced functional limitations) was positively correlated with depression. Similar findings were reported by Kennedy et al. (1995). In their study, also involving people with SCI, a positive association was found between functionality (more pronounced functional limitations) and psychosocial distress. In a sample of people with multiple sclerosis, Wineman (1990) found that those with more severe functional limitations (i.e., increased limitations in the ability to perform usual roles and activities) also experienced increased depression and a decreased sense of purpose in life. In their meta-analytic reviews of the literature on psychosocial adjustment among children and adolescents with physical disabilities, Lavigne and Faier-Routman (1992, 1993) reported that decreased functional status was negatively linked to measures of adjustment. Lastly, in a longitudinal study by Eide and Roysamb (2002), it was found that functional limitations, as reported by respondents with a wide range of disabling conditions (e.g., musculoskeletal, circulatory, respiratory, emotional), predicted psychosocial adjustment problems over a period of several years. Based on this review of the empirical literature, the current study investigates the hypothesized relation between functional limitations and adaptation-associated outcomes of college adjustment (i.e., life satisfaction, academic performance, psychosocial-emotional adjustment to college).

Coping and adaptation to college. In the adapted QoL-PACID model, 
intrapersonal coping (i.e., engagement-type coping) is investigated as a process-linked variable. The specific hypothesized relation predicts engagement-type coping strategies as significantly and positively related to adaptation-associated college adjustment outcomes (i.e., life satisfaction, academic performance, psychosocial-emotional adjustment to college) among first-year and second-year undergraduate students with with disabilities.

The research on coping with a stressful life event and measures of adaptation has been expanding at a rapid rate (Livneh \& Martz, 2012). In particular, research examining the role of coping strategies (situationally determined) in psychosocial adjustment to college has proliferated over the past several decades (e.g., Appelhans \& Schmeck, 2002; Aspinwall \& Taylor, 1992; Kariv \& Heiman, 2005; Folkman \& Lazarus, 1985; Leong et al., 1997; Mortenson, 2006; Struthers et al., 2000; Yum et al., 2005 ). Among the engagement-oriented strategies, the higher education literature reveals the investigations of both behavioral and cognitive aspects of coping. Frequently examined behavioral coping approaches include active planning, seeking social support, and information seeking (e.g., Aspinwall \& Taylor, 1992; Collins, Mowbray, \& Bybee, 1999; Kariv \& Heiman, 2005; Leong et al.,1997; Lopez, Mauricio, Gormley, Simko, \& Berger, 2001; Misra \& McKean, 2000; Mortenson et al., 2006; Pearlin, 1991; Solberg \& Villarreal, 1997; Yum et al., 2005). The more frequently investigated cognitive approaches include positive reinterpreting/appraising and cognitive restructuring (e.g., Lee, 2007; Kolchakian \& Sears, 1999; Mattlin et al., 1990). The higher education 
empirical literature supports engagement-type (both behavioral and cognitive) approaches as most effective in abating stress, and facilitating positive adaptive outcomes in postsecondary student samples (e.g., Appelhans \& Schmeck, 2002; Aspinwall \& Taylor, 1992), including students with disabilities (e.g.,Kariv \& Heiman, 2005; Sanders \& DuBois, 1996).

In contrast, commonly investigated disengagement coping strategies include denial, blaming, self-criticizing, avoiding/escaping problems, socially withdrawing, engaging in cognitive distraction, and using alcohol and/or drugs. These strategies have been consistently associated with poorer adaptive outcomes in a variety of postsecondary student populations (e.g., Aspinwall \& Taylor, 1992; Dyson \& Renk, 2006; Leong et al., 1997; Wodka \& Barakat, 2007), including postsecondary undergraduate students with disabilities (Wodka \& Barakat, 2007).

For example, a study investigating differential coping styles as predictors of college adjustment among 161 first-year students attending an Eastern Ivy League college was conducted by Leong et al. (1997). These researchers assessed college adjustment using Baker and Siryk’s (1999) Student Adaptation to College Questionnaire (SACQ). The SACQ measures four facets of college adjustment: academic adjustment, personal/emotional adjustment, social adjustment, and attachment/goal commitment. Coping, the predictor variable in this study, was assessed by Weintraub, Carver, and Scheier's (1986) COPE questionnaire. The COPE Questionnaire measures 12 conceptually derived scales which describe different ways 
of responding to stress. Each construct is operationalized by examples of specific behaviors, with questions asking individuals how often they use particular coping strategies. Results suggested that the "active," engagement-type coping strategy was predictive of both academic adjustment and psychosocial-emotional adjustment facets college adjustment. Furthermore, psychosocial-emotional adjustment to college was negatively related to "focus on and venting of emotions," an emotion-focused, disengagement response which consists of coping by emotional catharsis, a strategy that arguably is less useful in responding to stress.

Although empirical studies on coping with college-related stressors have been widely published over the past several decades, few studies have examined coping and adaptation to college in the context of students with disabilities (e.g., Kariv \& Heiman, 2005; Wodka \& Barakat, 2007).

For example, a study by Wodka and Barakat (2007) examined the role of coping in psychological adjustment to college among students with chronic illness and disability (CID group), compared with college students who experience predominantly negative life events (NLE group) or positive life events (PLE group). The sample consisted of 101 first-year and second-year undergraduate students attending an urban, East Coast University. Participants were placed in three groups: (a) those who experienced a CID $(n=32)$; (b) those who experienced predominantly NLE $(n=53)$, as measured by the Life Experiences Survey (Sarason, Johnson, \& Siegel, 1978); and (c) those who experienced predominantly PLE $(n=16)$, as measured by the Life 
Experiences Survey (Sarason, et al., 1978).

In this exploratory study, coping strategies were measured with the 60-item COPE Inventory (Carver et al., 1989). Scores on the active (i.e., engagement-oriented) and passive (i.e., disengagement-oriented) coping scales were used. The active scale consists of active coping, planning, suppression of competing activities, positive reinterpretation and growth, and restraint coping subscales. The passive scale consists of acceptance focus on venting of emotions, denial, mental disengagement, behavioral disengagement, and alcohol/drug use subscales.

Adaptational outcomes (i.e., adjustment to college) were measured by symptoms of anxiety and depression. Specifically, anxiety was assessed using the total symptoms scores of the Beck Anxiety Inventory (BAI) (Beck \& Steer, 1993). The BAI is a 21-item measure of anxiety in individuals over 17 years of age. To assess depression, the Beck Depression Inventory-II (BDI-II) (Beck, et al., 1996) was utilized. The BDI-II is a 21-item instrument that identifies symptoms associated with a DSM-IV diagnosis of major depressive disorder.

Correlational analysis indicated that passive coping was positively associated with poorer adaptational outcomes in all three groups. Specifically, when coping was used as the predictor variable, a significant correlation between passive coping (i.e., the denial of problems; mental and behavioral disengagement) and anxiety was noted in students with disabilities (i.e., CID group) $(r=.37, p=.036)$. In the NLE group, passive coping was significantly correlated with depression $(r=.47, p=.001)$. When the PLE group 
was examined, passive coping was significantly associated with depression $(r=.61$, $p=.012$ ). For all analyses, significance was considered with a $p$ value $<.05$. Although a significant limitation of this study was its small sample size, these results do add support for the role of disengagement-oriented strategies as least adaptive in times of stress, such as the transition to college among students with a chronic physical health condition.

Perceived social support and adaptation to college. In the adapted QoL-PACID model, perceived social support is conceived as a process-linked predictor variable. The specific hypothesized relation predicts perceived social support as significantly and positively related (i.e., direct, linear relationship) to adaptation-associated college adjustment outcomes (i.e., life satisfaction, academic performance, and psychosocial-emotional adjustment to college).

The concept of perceived social support has received considerable research attention in the education literature, particularly in regards to its direct link with adaptation-associated indices of college adjustment in first-year and second-year undergraduate students. College students who perceive supportive relationships (e.g., family, peers, and others) indicate higher levels of life satisfaction than students who are less satisfied with such support (e.g., Bean \& Bradley, 1986; Coffman \& Gilligan, 2002; Demarkis \& McAdams, 1994; Tinto, 1993). In addition, low levels of perceived support are predictive of poorer academic and psychosocial-emotional adjustment to postsecondary life among undergraduate students (e.g., Cohen \& Wills, 
1985; Coffman \& Gilligan, 2002; Elliott, Herrick, \& Witty, 1992; Felsten \& Wilcox, 1992; Halamandaris \& Power, 1999; Lamothe et al., 1995; Rodriguez et al., 2003; Sanders \& DuBois, 1996; Solberg et al., 1994; Solberg \& Villarreal, 1997; Wilkes \& Spivey, 2010; Wintre \& Yaffe, 2000; Yalcin, 2011). This also includes a research focus on the role of perceived support in college adaptation regarding under-represented student populations, such as minority students (e.g., African American, Chinese, Latino) (e.g., Mallinckrodt, 1988; Reifman \& Dunkel-Schetter, 1990; Solberg \& Villarreal, 1997; Tao et al., 2000; Zea et al., 1995), and students with various disabilities (i.e., physical, cognitive/neurocognitive, psychiatric) (e.g., Cosden \& McNamara, 1997; Winterowd et al., 1998; Wodka \& Barakat, 2007). Unfortunately, most studies exploring social support and its relation to adjustment outcomes in undergraduate students with disabilities, suffer from small sample sizes, thus affecting power and statistical rigor.

Engagement-type coping as a moderator between college stress and adaptation to college. The adapted QoL-PACID model views engagement-type coping as a process-linked variable that is hypothesized to moderate college stress in adaptation to college (as measured by life satisfaction, academic performance, and psychosocial-emotional adjustment to college) among first-year and second-year undergraduate students with disabilities.

Although no empirical studies could be located examining the moderating (i.e., interactive) role of engagement-type coping in a population of postsecondary 
students with CID, the stress-interactive effects of engagement-type coping strategies have been documented in past studies of the general college student population (e.g., Hovantz \& Kozora, 1989; Lee, 2007), as well as in ethnically diverse groups (e.g., Crockett et al., 2007; Zea, Jarama, \& Bianchi, 1995). On the basis of these studies, engagement-oriented coping strategies (e.g., problem solving, planning, positive reframing) are documented as playing a beneficial role in moderating stress in diverse college student populations.

For example, in a study by Crockett et al., (2007), the role of coping as a moderator of acculturative stress was investigated in a subpopulation of Latino (Mexican or Mexican-American origin) postsecondary students. The analytic sample consisted of 148 students ( $67 \%$ female; mean age $=23.05$ years, $\mathrm{SD}=3.33$ ), ranging 18 to 30 years in age, and decidedly Mexican-American (83\%) in origin. Nearly three quarters of those students participating in the study had one (26\%) or two (46\%) Mexican-born parents. The study was conducted at three state universities (one in Texas; two in California), with data collected during the summer session at the Texas university, and the academic year (i.e., fall and winter semesters) at both universities in California. Acculturate stress was measured by the Social, Attitudinal, Familial and Environmental Acculturative Stress Scale (Mena, Padilla, \& Maldonado, 1987), which measures stress in four domains: familial (e.g., "Close family members and I have conflicting expectations about my future"), attitudinal (e.g., "I often think about my cultural background"), social ("I don't feel at home"), and environmental ('it bothers 
me when people pressure me to assimilate"). Coping was measured with a shortened version of the COPE Inventory (Carver et al., 1989), (i.e., Brief COPE; Carver, 1997) which assesses different dimensions of active (i.e., problem-focused, engagementoriented) or avoidant (emotion-focused, disengagement-oriented) coping styles. The subscales were aggregated to form two composite scales: Active coping (items reflecting problem solving, planning, and positive reframing) and avoidant coping (items reflecting behavioral disengagement, mental disengagement/self-distracting, denial, and alcohol/substance use). Psychological adjustment to college was assessed using the total symptoms score of the 21-item Beck Anxiety Inventory (BAI) (Beck \&Steer, 1993), and total symptoms score of the Center for Epidemiological Studies Depression Scale (CES-D) (Radloff, 1977). Tests of interaction effects indicated that active coping moderated the effects of high acculturative stress on both symptoms of anxiety and depression, thus supporting the stress-interactive hypothesis in a postsecondary student sample.

Engagement-type coping as a moderator between functionality and adaptation to college. The adapted QoL-PACID model views engagement-type coping as a process-linked variable that is hypothesized to moderate functionality (as measured by increased restrictions in the ability to perform usual daily activities and life roles) in adaptation to college (as measured by life satisfaction, academic performance, and psychosocial-emotional adjustment to college).

The literature reveals the moderating role of intrapersonal coping, in attenuating 
impact of functionality (as measured by increased restrictions in the ability to perform daily activities and life roles) in adjustment to disability among postsecondary student samples. For example, a study by Haden et al (2006) revealed that functionality (as measured by perceived level of disability severity) significantly predicted levels of posttraumatic stress disorder (PTSD) symptoms in a nonclinical sample of 150 undergraduate college students who reported experiencing different types of trauma (i.e., car accidents, natural disasters, violent crimes, unwanted adult sexual experiences, childhood abuse, and abusive relationships). The number of years since respondents experienced the reported trauma ranged from a few months to 18 years, with an average time of 5 years and 6 months ( $\mathrm{SD}=4$ years, 5 months). Regarding the measurement of trauma exposure, respondents completed the Events Scale (ES) (Vrana \& Lauterbach, 1994). Level of perceived injury was measured by the question, "Were you injured?" and answered on a 7-point Likert-type scale ranging from "not at all" to "severely." Respondents' PTSD-related symptoms were measured by the Purdue Post Traumatic Stress Disorder-Revised Questionnaire (PPTSD-R) (Lauterbach \& Vrana, 1996). Coping responses were measured by the 60-item COPE Inventory (Carver et al., 1989). Hierarchical multiple regression revealed a significant moderating relationship: respondents who self-reported more severe levels of traumatic injury reported less severe PTSD symptoms when they utilized an active, intrapersonal style of coping (i.e., engagement-type strategy). The specific intrapersonal coping behavior consisted of asking advice for problem-solving). The study contributes to the growing body of 
literature examining the role played by intrapersonal coping (i.e., engagement-oriented strategies) as a moderator of a disability-related stressor (i.e., functional limitations). No empirical studies could be located however, examining the moderating (interactive) role of engagement-type coping strategies, in the relationship between functionality (as measured by increased restrictions in functional ability) and adaptation-associated indices of college adjustment among undergraduate students with CID, the focus of the current study.

Perceived social support as a moderator of college stress in adaptation to college. The adapted QoL-PACID model considers perceived social support as a process-linked variable that is hypothesized as a moderator between college stress and adaptation to college (as measured by life satisfaction, academic performance, and psychosocial-emotional adjustment to college) among first-year and second-year undergraduate students with disabilities.

Evidence for perceived social support's role in moderating the impact of stressful events can be found in Sek's (1991) investigation into the relation between perceived social support and second-year undergraduate college students' coping with life stress. In particular, Sek found that perceived social support from family and friends acted as a protective buffer against stressful events reported on the College Student Life Events Schedule (Harrari, Jones, \& Sek, 1988), thereby reducing the appraisal of stress. Perceived social support especially acted as an effective moderator when the stress was very intense, demonstrating that the role of perceived social support depends on the type 
and source of support, and on appraisals of the effectiveness of the support.

Perceived social support as a moderator of functionality in adaptation to college. The adopted Qo-PACID model considers perceived social support as a process-linked variable that is hypothesized as a moderator between functionality (as measured by increased restriction in the ability to perform in daily activities and life roles) and adaptation-associated adjustment to college outcomes (i.e., life satisfaction, academic performance, psychosocial-emotional adjustment to college) among first-year and second-year undergraduate students with disabilities.

Moderating effects of perceived social support have been found for a wide variety of perceived negative live events, including functionality (i.e., more pronounced functional limitations). However, such studies could only be located from the rehabilitation psychology literature as pertaining to adjustment to disability (rather than adjustment to college among students with disabilities). If perceived social support operates as a moderator, then the negative impact of functionality should be reduced for persons who report having a high level of perceived social support. Indeed, a study by Littlefield, Rodin, Murray, and Craven (1990) examined the stress-interactive effects of perceived social support on depression among a non-college sample of individuals with Type I diabetes. Analysis indicated a Support x Functionality interaction effect: Functionality (illness-related impairment) was strongly related to depression among persons with inadequate perceived support, but the effect of functionality was considerably reduced for persons with adequate perceived social support. Empirical 
evidence thus supports that the perception of social support plays an interacting (i.e., moderating) role in the relationship between functionality and positive adaptive outcomes (i.e., lower level of depression) in persons with a disability.

A study by Haden et al (2006) (i.e., previously discussed) assessed whether perceived social support moderated the relationship between functionality (as measured by perceived injury severity) and adjustment to disability (as measured by PTSD symptoms) in a population of undergraduate college students. The sample consisted of 150 students ( $n=50$ male; $n=100$ female), ranging 17 to 22 years in age $(M=19.33$; $S D=1.31$ ), and who were primarily Caucasian. Participants reported experiencing a range of traumas, including technological accidents (e.g., car accidents), natural disasters, violent crimes, unwanted adult sexual experiences, childhood abuse, and abusive relationships. The number of years since respondents experienced the reported trauma ranged from several months to 18 years ( $M=5$ years, 6 months; $S D=4$ years, 5 months). Regarding trauma exposure, respondents completed the Events Scale (ES; Vrana \& Lauterbach, 1994). The ES is a self-report measure that assesses respondents' exposure to various types of stressful events. In addition, respondents reported different aspects of the trauma including when it had occurred, how traumatic it had been, and how much perceived injury had been sustained. Level of perceived injury was measured by the question, "Were you injured?" and was answered on a 7-point Likert type scale ranging from "not at all" to "severely." Respondents indicated sustained injury levels ranging from 0 to $7(M=1.80 ; S D=1.37)$. PTSD-related symptoms were measured by 
responses on the Purdue Post Traumatic Stress Disorder-Revised questionnaire (PPTSD-R; Lauterbach \& Vrana, 1996). Respondents were instructed to respond based on the most traumatic event they had experienced. The PPTSD-R includes 17 items that comprise three scales: (1) re-experiencing the trauma, (2) avoidance, and (3) arousal based on DSM-IV symptomatology for PTSD. Each item was answered on a 5-point Likert type scale ranging from "not at all" to "often" regarding the frequency of each symptom during the previous month. The cumulative PTSD severity score was calculated and ranged from 17 to $69(M=30.31 ; S D=13.34)$.

Perceived social support was assessed with the Multidimensional Scale of Perceived Social Support (MSPSS; Zimet, Dahem, Zimet, \& Farley, 1988).

Respondents rated their agreement with four statements pertaining to the perceived support received from family, and four statements referring to the perceived support received from friends, on a 7-point Likert scale ranging from "very strongly disagree" to "very strongly agree." Mean scale scores were used for family and friend support. Family $(M=19.19 ; S D=5.24)$ and friend support $(M=19.32 ; S D=4.84)$.

Individuals who reported higher levels of functionality (increased restrictions in ability to perform daily activities and life roles) reported less severe PTSD symptoms when they perceived higher levels of support from friends. Consistent with previous research, a significant interactive effect for perceived social support on the relationship between perceived injury and PTSD symptoms was supported. The concept of supportive social relations may therefore be considered an important moderating 
verbal for minimizing the severity of PTSD in young adults. 


\section{Chapter III}

\section{Methodology}

In this chapter the researcher describes the methods used to investigate relationships proposed to influence college adjustment, in first-year and second-year undergraduate students with disabilities. Predictor variables included one college-related (i.e., college stress, as measured by the College Stress Inventory [CSI; Solberg et al., 1993] and one disability-related (i.e., functional limitations, as measured by the Disability Functional Limitations Scale [DFLS]) indices, as well as two personal coping (i.e., engagement coping, measured by the Brief COPE [Carver et al., 1993], and perceived social support, measured by the Social Support Appraisals Revised scale [SSA-R; Vaux et al., 1986]) indices. The potential moderating (i.e., interacting) role of engagement coping and perceived social support (each) were also explored. Adjustment to college was assessed by three criterion measures reflecting a QoL perspective of adaptation, and included: (a) satisfaction with life (SWLS; Diener, Emmons, Larson, \& Griffin, 1985) (b) academic performance (GPA-S), and (c) psychosocial-emotional adaptation to college (SACQ; Baker \& Siryk, 1999). In addition, the discussion in this section of the dissertation will focus on: (a) research aims/statistical analysis, (b) sample, (c) measures, and (d) procedures for data collection.

\section{Research Aims/Statistical Analysis}

Three specific research aims were included in this study, along with one sub-aim. The first aim was concerned with testing hypothesized relationships between the study's 
four predictor (i.e., college stress, functional limitations, engagement coping, perceived social support) and three adaptation-associated criterion variables (i.e., life satisfaction, academic performance, and psychosocial-emotional adjustment to college). Hoyt, Leierer, and Millington (2006) advocate the use of bivariate correlation analyses when testing hypotheses of linear associations among variables. Thus, bivariate (i.e., single-predictor) correlation analyses was employed to examine hypothesized relations addressing the first aim of the study (i.e., hypotheses 1 through 4 ).

This study's second aim was concerned with testing hypothesized relationships between predictor (i.e., college stress; functional limitations) and potential stress moderating variables (i.e., engagement-type coping strategies; perceived social support), and the study's three measures of adaptation to college (i.e., life satisfaction; academic performance; psychosocial-emotional adjustment to college). A moderator variable is one that influences the relationship between a predictor variable and a criterion variable by affecting the strength or direction of the relationship (Baron \& Kenny, 1986; Holmbeck, 1997). To assess the study's hypothesized moderating effects, the regression with interaction procedure described by Baron and Kenny (1986) was used. First, the predictor and moderator main effects are regressed on the criterion variable. Second, the interaction term representing the product of the two main effects (i.e., Engagement-Coping X College Stress; Engagement-Coping X Disability Functional Limitations; Perceived Social Support X College Stress; Perceived Social Support X Disability Functional Limitations) are entered into the equation. The 
moderator hypothesis is supported when the interaction term is significant.

More specifically, the second research aim of the study, testing a moderating role for engagement-type coping and perceived social support (each) was addressed by conducting a four-step multiple regression analysis: In step one, relevant socio-demographic variables are entered into the model. In step two, the unique contribution of each predictor variable are entered. These predictor variables included: college stress, as measured by the College-Stress Inventory (i.e., CSI; Solberg et al., 1989), and disability-related functional limitations, as measured by the Disability Functional Limitations Scale (i.e., DFLS; developed by the researcher). In step three, each potential moderator variable (i.e., Engagement-Coping; Perceived Social Support) is entered (in separate regressions). Lastly, in step four, the interaction term (i.e., Engagement-Coping X College Stress; Engagement-Coping X Disability Functional Limitations; Perceived Social Support X College Stress; Perceived Social Support X Disability Functional Limitations) is entered. Moderation is deemed present when the interaction term is significant (i.e., <.05). An interaction found to be significant is subjected to additional analysis in order to identify the specific conditions under which the moderator affects the relationship between the predictor and the criterion.

The third aim of the study was to investigate several socio-demographic variables (i.e., person-related [respondents chronological age, gender], vocation-related [hours employed per week while attending college], and disability-related [duration of 
disability, disability group membership]), and their possible relationships to the study's three criterion measures of college adjustment.

As a sub-aim, the study also investigated the role of disengagement-type coping strategies as possibly contributing to the variance in adaptation to college (as measured by life satisfaction, academic performance, and psychosocial adjustment to college), after controlling for relevant socio-demographic variables.

\section{Sample}

The population of interest for this research involved male and female first-year and second-year undergraduate students with disabilities, who were at least 18 years of age, and who volunteered to participate. In order to detect medium effect size $\left(R^{2}=.15\right)$ for multiple regression analysis (MRA), a power analysis was conducted, and determined to be: $N=85$ (for $\alpha=.05$; power $=.80 ; 4$ predictors). Varying the number of predictors from 5 to 8 variables, suggested a sample size in the range of 80 to 98 respondents. Power analysis, therefore, confirmed that a non-probability sample in the range of 80 to 98 persons was needed from the database of registered first-year and second-year undergraduate students with disabilities. These students will have attended either Portland State University or Oregon State University during fall, winter, spring, and/or summer term(s) of the 2008-2009 academic year. Potential participants were recruited by having the Director (or their staff) of each respective Disability Service Office send an announcement with cover letter by e-mail. Participants had to be able to read and write the English language. They also needed to have access to the internet, 
and a current e-mail address. First-year and second-year students (during the academic year of 2008-2009) who were no longer currently registered for classes, were also

welcomed to participate in the study. Those excluded from the study were first-year and second-year undergraduate students registered for classes with temporary disabilities (i.e., a condition expected to resolve within six months, such as a broken leg or impacted/infected wisdom tooth).

\section{Measures}

Measures used to obtain data pertinent to this study included:

Socio-demographic characteristics. The Participant Survey, developed by the researcher, is a self-administered instrument used to collect socio-demographic data and includes questions on respondents' age, gender, ethnic background, marital status, years since diagnosis of disability, primary disabling condition, type of housing, current enrollment status, the number of hours per week employed, academic level (i.e., firstyear or second-year), and cumulative grade point average.

College Stress. A modified version of the 21-item College Stress Inventory (Solberg et al., 1993) will be used to assess experienced college-related stress. The CSI contains three subscales: Academic stress, social stress, and financial stress. Academic stress subscale items address issues such as preparing for exams, meeting with faculty, and asking questions in class. The social stress subscale consists of two sub-factors; stress stemming from issues related to ethnicity (or in the case of the present study, stress stemming from issues related to disability), and stress resulting from issues 
related to interpersonal competence. Items from the social stress subscale address issues associated with being at college, and especially issues related to one's ethnicity (or in the case of the present study, disability), such as feeling as though one is treated differently by peers and faculty. Financial stress items address various issues related to the student's economic situation, including stress felt from family related to financial difficulty. Individuals rate each item on a 4-point Likert-type scale from $0=$ never to $4=$ always. Scores on the total scale range from 0 to 83 , with higher scores indicating greater college stress. Lower total CSI scores have been shown to predict greater well-being (Solberg \& Villarreal, 1997).

In regards to item modification, the word ethnicity was changed to disability, which has application for students with disabilities. This change was applied only for three items: "Difficulty trying to meet peers with your ethnicity" (item 1, social stress subscale); “difficulty from faculty on the basis of your ethnicity' (item 3, social stress subscale); and, "difficulty from peers on the basis of your ethnicity' (item 4, social stress subscale). Although permission for said modifications was sought, the primary author (i.e., Solberg) could not be located for consultation.

Internal consistency reliability for the total CSI scale has been reported at .89 (Solberg et al., 1993). The three subscales have been found to possess adequate internal consistency reliability (ranging from 0.82 to 0.88 ) (Solberg et al., 1993). Internal consistency reliability for the total CSI scale has been reported by Solberg et al. (1993) as 0.89 . No data have been reported on test-retest reliability however. Reliability in the 
current study was assessed using Cronbach's $\alpha$. In this sample $(N=103)$, internal consistency for the subscales included: academic stress $(\alpha=0.87)$, social stress ( $\alpha=0.86)$, and financial stress $(\alpha=0.91)$. The total CSI scale reflected a Cronbach's $\alpha$ of 0.91. A Cronbach's $\alpha$ of 0.80 indicates very good reliability for an instrument (Field, 2009).

A factor analysis on the CSI was conducted so as to minimize any redundant variance shared by the three subscales (i.e., academic, social, financial). Principal components analyses (PCA's) were conducted for participants scores on each of the three subscales. To ensure the factorability of the correlation matrix, Bartlett's test of sphericity was applied to the $3 \times 3$ correlation matrix. A significant chi-square value of $46.58(p<.001)$ supported continuation of the PCA. Before extraction, the analysis identified 3 linear components within the data set. The first factor, the CSI academic subscale, accounted for $60.63 \%$ of the pre-rotation variance (eigenvalue $=1.82$ ). The second factor, the CSI social subscale, accounted for $21.65 \%$ of the pre-rotation variance (eigenvalue $=.649$ ). The third factor, the CSI financial subscale, accounted for $17.73 \%$ of the pre-rotation variance (eigenvalue $=.532$ ). The CSI social subscale, and the CSI financial subscale, respectively, failed to meet an eigenvalue (a measure of explained variance) greater than 1.0, which is an essential criterion for a factor to be useful (Field, 2009). In addition, because of highly correlated subscales (i.e., multicolinearity among the subscales), it was determined that a single, summative score would best represent this variable. For purposes of this study, then, only the total 
score (i.e., summated scores for those items making up each factor) of the CSI was included in further analyses.

Functionality. The Disability Functional Limitations Scale, developed by the researcher, is a self-administered instrument designed to measure disability-related functional limitations. The degree of functional limitations will be measured by seven items that require participants to indicate on a 5-point scale $(1=$ not restricted at all, $2=$ only minimally restricted, $3=$ moderately restricted, $4=$ severely restricted, $5=$ totally restricted $)$ the degree to which their ability to function within their own environment is restricted by their disability. The responses are then summed up separately for each item to yield seven individual functionality scores. Individual scores are then added up for a total DFLS score, with higher scores indicating more severe functional limitations. Included items were selected based on activities and participation (two dimensions of disability in terms of the second edition of the International Classification of Functioning, Disability and Health [ICFDH-2] [World Health Organization, 2001]) in relevant domains of functioning in which a university student would be engaged in daily life. The ICFDH-2, more commonly known as the ICF, is a model of functioning and disability that systematically organizes the consequences of disease into three dimensions: (a) body functions and structure (symptoms and impairment), (b) activities, and (c) participation. The ICF model acknowledges that the settings in which individuals live their lives play a central role in the expression of their capacity to function (Stucki, Cieza, \& Melvin, 2007). Several 
studies support the ICF as a useful framework for the assessment of functioning and disability in chronic conditions (Bruyere, Van Looy, \& Peterson, 2005; Chopra, Couper, \& Herman, 2002; Chwastiak \& Von Korff, 2003; Fransen et al., 2002; Jelsma, 2009).

Perceived Social Support. Perceived social support will be measured with the Social Support Appraisals (SSA) scale (Vaux et al., 1986). The SSA is a 23-item instrument based on the assumption that social support is in fact support only if the individual believes it is available. These subjective appraisals also are viewed as related to overall psychological well-being. The SSA taps the extent to which the individual believes he or she is loved by, esteemed by, and involved with family, friends, and others.

The SSA is scored by reverse scoring items $3,10,13,21,22$ and adding up the individual items for a total score, with lower scores indicating a stronger subjective appraisal of social support. In addition to the total score, the seven family items make up an SSA-family subscale, and the seven friend items make up a friend subscale. The remaining nine items refer to people or others in general. Because the SSA is scored such that the lower the score the better the level of perceived social support, and the present study's three criterion measures (i.e., SWLS; GPA-S; SACQ) are scored such that the higher the score the better the adjustment, each item of the SSA was reversed scored (i.e., the higher the score the greater level of perceived social support). This resulted in a renamed instrument: Social Support Appraisal-Revised (i.e., SSA-R) scale. The original SSA (Vaux et al., 1986) has very good internal consistency, with $\alpha$ 
coefficients that range from 0.81 to 0.90 . Internal consistency in the current study sample $(N=103)$ is 0.96 , which indicates excellent reliability (Field, 2009) for the total SSA-R scale. The original SSA has been subjected to considerable evaluation of its validity, resulting in data that support very good concurrent, predictive, known-groups, and construct validities (O’Reilly, 1995; Procidano \& Heller, 1983). The SSA is significantly correlated in predicted ways with a variety of measures of social support and psychological well-being, including network satisfaction, perceived support, family environment, depression, positive affect, negative affect, loneliness, life satisfaction, and happiness (Vaux et al., 1986).

A factor analysis was conducted on the SSA-R so as to minimize any redundant variance shared by the three subscales (i.e., family, friend, other). Principal components analyses (PCA's) were conducted for participants scores on each of the 3 subscales. To ensure the factorability of the correlation matrix, Bartlett's test of sphericity was applied to the 3 X 3 correlation matrix. A significant chi-square value of $266.09(p<.001)$ supported continuation of the PCA. The first factor, the SSA-R family subscale, accounted for $88.53 \%$ of the pre-rotation variance (eigenvalue $=2.66$ ). The second factor, the SSA-R friend subscale, accounted for $7.60 \%$ of the pre-rotation variance (eigenvalue $=.228)$. The third factor, the SSA-R other subscale, accounted for $3.86 \%$ of the pre-rotation variance (eigenvalue $=.116$ ). The SSA-R friend subscale, and the SSA-R other subscale, respectively, failed to meet an eigenvalue greater than 1.0, which is an essential criterion for a factor to be useful (Field, 2009). In addition, because of 
highly correlated subscales (i.e., multicolinearity among all three subscales), it was determined that a single, summative score would best represent this variable. For purposes of this study, then, only the total SSA-R score (i.e., summated scores for those items making up each factor) was included in further analyses.

Coping. Coping strategies will be measured with the 28-item Brief Coping Orientations to Problems Experienced (Brief-COPE; Carver, 1997). The original COPE Inventory (Carver, et al., 1989) consisted of 14 scales, each focusing on a unique coping strategy (a later version included 15 scales, with the addition of humor as a coping strategy). Each of the strategies consists of four items, with the exception of the alcohol-drug disengagement scale, which is typically measured by a single item. Respondents are instructed to indicate how they generally feel and what they generally do when experiencing a stressful event.

Items on the COPE Inventory are endorsed on a 4-point scale, ranging from 1 ("I usually do not do this at all") to 4 ("I usually do this a lot"). The responses are then summed up separately for each scale to yield 14 or 15 individual coping scores. Carver et al. (1989) reported that test-retest reliability coefficients for the 14 scales, over a 2-month period, ranged from 0.42 to 0.89 , with a median stability reliability coefficient of 0.63. Internal (homogeneity) reliability estimates (Cronbach's alpha) ranged from 0.45 to 0.92 , with a median coefficient of 0.71 . Inter-scale correlations among the 14 scales ranged from -0.28 to 0.69 .

For the purposes of this study, an abbreviated form of the COPE Inventory will be 
used (Brief COPE; Carver et al, 1993). Carver et al. (1993) reduced the number of items to two per scale and used the abbreviated form to measure coping among women with early-stage breast cancer. The Brief COPE measures a broad range of cognitive and behavioral coping strategies that individuals typically use in stressful situations. It includes 28 items, which measure 14 conceptually distinct facets of coping: namely, active coping (i.e., "I've been taking action to try to make the situation better"), planning (i.e., "I've been thinking hard about what steps to take"), positive reframing (i.e., "I've been looking for something good in what is happening"), acceptance (i.e., "I've been learning to live with it"), humor (i.e., "I've been making jokes about it"), religion (i.e., "I've been praying or meditating"), using emotional support (i.e., "I've been getting emotional support from others"), using instrumental support (i.e., "I've been getting help and advice from other people"), self-distraction (i.e., "I've been turning to work or other activities to take my mind off things"), denial (i.e., "I've been saying to myself, 'this isn't real"), venting (i.e., "I've been expressing my negative feeling"), substance use (i.e., "I've been using alcohol or other drugs to help me get through it"), behavioral disengagement (i.e., "I've been giving up the attempt to cope"), and self-blame (i.e., "I've been criticizing myself"). Despite the fact that the 14 scales are only two items each, their reliabilities all meet or exceed the value of .50, regarded by Nunnally (1978) as minimally acceptable psychometric values (Carver, 1997).

Coping theory proposes that the 14 coping strategies assessed by the Brief COPE (Carver, 1993) are part of several larger constructs (e.g., problem-focused, 
emotion-focused, approach, engagement, avoidance, regressive, and disengagement coping) (Carver et al., 1989). However, researchers have warned against the practice of assuming that certain coping strategies are always grouped in the same way across different contexts (Lazarus \& Folkman, 1984). Therefore, a factor analysis was conducted in the current study to see how the subscales grouped together to form broader coping constructs in this sample of college students with disabilities $(N=103)$. Factor analysis also minimizes any redundant variance shared by the 14 separate coping strategies. Principal components analysis (PCA) was conducted for participants scores on each of the 14 coping scales. To ensure factorability of the correlation matrix, Bartlett's test of sphericity was applied to the 14 x 14 correlation matrix. A significant chi-square value of $1008.51(p<.001)$ supported continuation of the PCA. The varimax-rotated procedure with Kaiser Normalization for the data indicated a three-factor solution. This solution was retained because it was further supported by three factor retention criteria. Namely, eigenvalue larger than 1 (1.33), the Scree test, and interpretability of results. The three factor solution cumulatively accounted for $70.22 \%$ of the variance in the obtained data. Only scores (i.e., summated item scores for those items making up each factor) on the first two coping factors (i.e., engagement-type; disengagement-type, respectively) were included in further however. It was decided that for the purposes of this study it would be more parsimonious to focus on engagement and disengagement coping factors for two reasons. First, these two scales explained a higher portion of the resultant coping 
variance in this sample (accounting for $60.71 \%$ of the variance) and secondly, they appeared to be consistent with current notions of the structure of coping as identified by Tobin et al. (1989). Namely, the coping dimensions of engagement-type and disengagement-type coping strategies.

The first factor, engagement-oriented coping, accounted for $48.24 \%$ of the pre-rotation variance (eigenvalue $=6.75$ ), and included five coping scales (Active Coping, Planning, Positive Reframing, Spiritual-Religious, and Seeking Social Support-Instrumental). These scales suggest cognitive, social, and behavioral efforts at engaging the problematic event. The second factor, disengagement-oriented coping, accounted for $12.47 \%$ of the pre-rotation variance (eigenvalue $=1.75$ ), and consisted of seven coping strategies (Denial, Mental Disengagement, Emotional Venting, Seeking Social Support-Emotional, Blaming [Self or Others] Alcohol-Substance Abuse, and Behavioral Disengagement). These approaches are indicative of cognitive, social, and behavioral disengagement from stressful situations. The internal consistencies of these two factors were adequate for the current study; engagement-type coping $(\alpha=.081)$ and disengagement-type coping $(\alpha=0.90)$, respectively.

Psychosocial Adaptation to College. Psychosocial adaptation was measured with one global measure of life satisfaction (The Satisfaction with Life Scale [SWLS; Diener et al., 1985]), and one college specific measure (Student Adaptation to College Questionnaire [SACQ; Baker \& Siryk, 1999]). In addition, academic performance was assessed by a GPA-derived measure adapted by the researcher (located on the 
Participant Survey) that assessed the level of a student's self-reported cumulative GPA, based on a 4 point scale (i.e., Grade Point Average-Scale [GPA-S]).

Global measure of life satisfaction. The Satisfaction With Life Scale (SWLS)

(Diener et al.,1985) contains five items and will be used to assess quality of the participant's life as a whole. The SWLS assesses cognitive, rather than affective processes of well-being (Diener et al., 1985). Each item is rated on a Likert-type response format ranging from 1, strongly disagree, to 7, strongly agree. On the SWLS, individuals are asked such questions as whether their life is close to their ideal or whether they would describe the conditions of their life as excellent. Higher scores reflect greater subjective well-being. Acceptable reliability and construct validity have been demonstrated. Pavot, Diener, Colvin, and Sandvik (1991) reported coefficient alphas of 0.83 and 0.85 in two separate studies. Diener et al. (1985) reported a coefficient alpha of 0.87 and a two-month test-retest stability coefficient of 0.82 . In the current study, internal consistency (i.e., Cronbach's alpha) is found to be adequate (i.e., $\alpha=.83$ ). Correlates with other instruments indicate that the scale is relatively independent of social desirability effects and psychopathology, and it is favorably associated with other measures of life satisfaction (Diener et al., 1985).

College-specific measure. The Grade Point Average-Scale (GPA-S) is a college-specific measure of academic adjustment to college. The GPA-S (located on the Participant Survey) was derived by the researcher as an extension of the customarily-used GPA measure (i.e., index). Scores range from 1 (1.66 or below 
[D average or below]) to 8 (4.00 to 3.67 [A to A- average]). A higher numerical score is associated with a higher level of academic adjustment. Only one score is reported. For example, a respondent with a self-reported 3.20 cumulative GPA will score 6 on the derived measure (i.e., 3.32 to 3.00 [at least a B average, but just below a B+ ]).

College-specific measure. The last college-specific measure, designed to assess student psychosocial-emotional adjustment to college, is the Student Adaptation to College Questionnaire (SACQ) (Baker \& Siryk, 1999). The SACQ is a 67-item, self-report questionnaire that yields scores for overall adaptation to college as well as four facets of college adjustment: (a) academic (e.g., "I have been keeping up-to-date on my academic work"), (b) social (e.g., "I am very involved with social activities in college"), (c) personal-emotional (e.g., "I have been feeling tense or nervous lately"), and (d) goal commitment-institutional attachment ("e.g., "Getting a college degree is very important to me"). Each item is rated on a 9-point Likert scale ( $1=$ "applies very closely to me" to $9=$ "doesn't apply to me at all"). The total adjustment score is the sum of the ratings for all 67 items. Subscale scores are derived by summing the ratings for the items comprising each subscale. Items are coded such that higher scores on each scale are indicative of more positive adjustment ratings in that domain. To prevent an unwieldy amount of data, the current study reported (per participant) only the total SACQ score. Internal consistency reliability for the total SACQ scale was conducted for the current sample $(N=103)$ and reflected a Cronbach $\alpha$ of 0.91 . According to Field (2009), a Cronbach's $\alpha 0.90$ indicates excellent reliability for an instrument. Extensive 
reliability data for all subscales, as well as total SACQ scale data, have been reported by Baker and Siryk (1999), suggesting that the SACQ is a reliable measure of college adjustment. Data for first-year (i.e., freshmen-level) samples at three postsecondary institutions, for example, have shown high internal reliability (coefficient $\alpha$ ) for all scales: academic ( 0.81 to 0.90$)$, social (0.83 to 0.91$)$, personal-emotional ( 0.77 to 0.86 ), and attachment ( 0.85 to 0.91$)$. Criterion-related validity has been demonstrated through significant correlations between scores on the SACQ and student attrition, appeals for psychological services, and grade point average (GPA). Specifically, lower scores on all scales were found to be associated with increased rates of student attrition, whereas a significant positive correlation was found between GPA and scores on the academic scale, and a significant negative correlation was found between scores on the personal-emotional scale and students' efforts to obtain psychological counseling.

\section{Procedures for Data Collection}

Review and approval of this study was completed by Portland State University's: (a) Graduate School of Education, Doctoral Program, (b) Human Subjects Research Review Committee, and (c) Dissertation Proposal Committee.

The Director of Portland State University's Disability Resource Center (Polly Livingston), as well as the Director of Oregon State University's Disability Services Office (Dr. Tracy Townsend) were contacted concerning the research. The purpose of the study was explained to both Directors, and permission in contacting potential participants for the study was obtained. Students who agreed to participate: (a) read an 
e-mail containing a cover letter, (b) were informed as to the general nature and purpose

of the study, (c) had any questions about the research answered, and (d) were also asked to give their informed consent by clicking the response: "I have read the consent statement and am ready to begin the survey." Study participants then clicked a link to begin the web-survey.

Participation in the study would not in any way prejudice any future relations with Portland State University or Oregon State University. Participants were able to withdraw from the study at any time, and without penalty of any kind. 


\section{Chapter IV}

\section{Data Analysis}

This chapter is presented in five sections. The first section denotes procedures used to prepare data for analyses. In the second section, descriptive statistics that include zero-order correlations are presented. The third section presents the outcomes of data analyses related to the eight hypotheses tested. This section is subdivided by those hypotheses using bivariate analyses (i.e., hypotheses 1 through 4) to analyze relationships between predictor variables and the study's three QoL indices of college adjustment, and those hypotheses using hierarchical multiple regression analyses (MRAs) (i.e., hypotheses 5 through 8) to test interaction (i.e., moderator) effects between predictor variables and the study's three QoL indices of adjustment. The fourth section presents the outcomes of data analysis related to the five research questions. Research questions 1 through 4 used bivariate correlation analyses to examine relationships between variables. In addition, research question 4 used an independent sample t-test to investigate possible mean score differences in terms of gender and the study's three adjustment outcomes. Question 5 used analyses of variance (i.e., ANOVA) to investigate possible disability group differences and their potential relationship to the study's three QoL indices of adjustment. Finally, in section five, the sub-aim of the study is presented. In this last section, hierarchical MRAs were used to examine whether or not disengagement coping contributes to the prediction of the study's three criterion measures of adjustment (i.e., satisfaction with life, academic 
performance, psychosocial-emotional adjustment to college), after controlling for relevant socio-demographic variables.

\section{Preparation of Data for Analysis}

The preparation of data for statistical analysis includes a brief review of the instruments selected to measure each predictor (i.e., college stress, functionality, coping, perceived social support) and criterion variable (i.e., life satisfaction, academic performance, psychosocial-emotional adjustment to college) in the adapted QoL-PACID model. This section of the chapter includes a brief description for correcting any missing data, and meeting parametric assumptions for further data analyses.

\section{College Stress Inventory (CSI)}

The College Stress Inventory (CSI; Solberg et al., 1993) was administered to measure college stress as a predictor variable in the adapted QoL-PACID model. The CSI measures three domains/facets of college stress: academic stress (i.e., "Difficulty meeting deadlines for course requirements"), social strain (i.e., "Difficulty finding support groups sensitive to your needs"), and financial stress (i.e., "Difficulty paying rent/housing"). As recommended by Solberg and colleagues (1993), corrections for missing data were made on a domain (i.e., individual subscale) basis, with the missing value replaced with the participant's mean score for that domain. This procedure eliminated missing values if no more than 1 item was left unanswered in a domain. Responses were summed for a domain sub-score, as well as an overall or total CSI 
score. Higher scores signify higher levels of college stress.

Data entry on the CSI were verified by comparing visually the numbers on a printout of the SPSS data file with the codes on the original web-survey source. After the record was verified, data cleaning were accomplished by using frequency distribution to check for outliers and any erroneous codes.

SPSS analyses were applied to determine whether assumptions of normality, linearity, and homoscedasticity were met for the CSI. There was a normal distribution of data, and assumptions of linearity and homoscedasticity were met. Further analysis of data was therefore deemed appropriate (Field, 2009).

\section{Disability Functional Limitations Scale (DFLS)}

The Disability Functional Limitations Scale (DFLS) is a self-administered instrument developed by the researcher to measure functionality (increased restrictions in the ability to perform usual daily activities and life roles, associated with college life), a predictor variable in the adapted QoL-PACID model of adaptation to college. Level of functionality is measured by seven items that require participants to indicate on a 5-point scale, ranging from 1 ("not restricted at all') to 5 ("totally restricted"), the degree to which their ability to function within their own environment is restricted by their disability. Included items were based on "activities' and "participation" (two dimensions of disability in terms of the second-edition of the International Classification of Functioning Disability and Health [ICFDH-2] [World Health Organization, 2001]) in relevant domains of functioning in which a university student 
would be engaged in daily life.

Corrections for missing data on the DFLS were made on an individual score basis, with the missing value replaced with the participant's mean score. This procedure eliminated missing values if no more than 1 item was left unanswered on the instrument. All seven responses were then summed for an overall or total DFLS score, with higher scores indicating a higher degree of disability-related functional restrictions.

Data entry on the DFLS were verified by comparing visually the numbers of a printout of the SPSS data file with the codes on the original web-survey source. After the record was verified, data cleaning were accomplished by using frequency distribution to check for outliers and any erroneous codes.

SPSS analyses were applied to determine whether assumptions of normality, linearity, and homoscedasticity were met for the DFLS. There was a normal distribution of data, and assumptions of linearity and homoscedasticity were met. Further analysis of data was therefore deemed appropriate (Field, 2009).

\section{Brief COPE}

The 28-item Brief COPE (Carver, 1997) was administered to assess coping strategies. The 14 subscales (two items for each subscale) were aggregated to form two composite scales: Engagement-type coping (five items reflecting problem solving, planning, and positive reframing), and Disengagement-type coping (seven items reflecting behavioral disengagement, mental disengagement/self-distracting, denial, and alcohol/substance use). Both composite scales were also abbreviated: Engagement-type 
coping was coded as E-COPE, and disengagement-type coping was coded as D-COPE. Respondents indicated how frequently they used each coping strategy on a frequency scale ranging from 1 (never) to 5 (always).

In the adapted QoL-PACID model, engagement-type coping (i.e., E-COPE) served as a predictor and possible moderator variable, while disengagement-type coping (i.e., D-COPE) served as a predictor variable. Corrections for missing data on the Brief COPE were based on guidelines provided by Tabachnick and Fidell (2012). These authors advise that although there is no definitive agreement as to an acceptable response rate for the analysis of data for a variable, $80 \%$ is considered a commonly used rate. Therefore, greater than $20 \%$ missing data on the Brief COPE was set as the upper bound for non-response. In the current study, six cases were deemed to have excessive missing data, and were subsequently removed for consideration of further analyses. All other missing data was corrected by assigning the value of the average item score for each composite scale (i.e., Engagement Coping [E-COPE]; Disengagement Coping [D-COPE].

Data entry on the Brief COPE were verified by comparing visually the numbers on a printout of the SPSS data file with the codes on the original web-survey source. After the record was verified, data cleaning were accomplished by using frequency distribution to check for outliers and any erroneous codes.

SPSS analyses were applied to determine whether assumptions of normality, linearity, and homoscedasticity were met for the Brief COPE. The diagnostic screening 
procedures revealed that these data met the assumptions of a multiple linear regression. That is, there was a normal distribution of data, and assumptions of linearity and homoscedasticity were met. Therefore, further analysis of data was deemed appropriate (Field, 2009).

\section{Social Support Appraisals-Revised Scale (SSA-R)}

The Social Support Appraisal-Revised (SSA-R) scale was administered to participants in order to measure the perception of social support. Perceived social support served as a predictor and possible moderator variable in the adapted QoL-PACID model. Missing data on the SSA-R were managed as directed by (Vaux et al., 1986). Corrections for missing data were made on a domain (i.e., 3 facets of perceived social support: peers, family, and others) basis by assigning the value of the average item score for that domain. This procedure eliminated missing values if no more than 1 item was left unanswered in each domain. Responses were summed to obtain an overall or total SSA-R score. Higher scores signify higher levels of perceived social support.

Data entry on the SSA-R were verified by comparing visually the numbers on a printout of the SPSS data file with the codes on the original web-survey source. After the record was verified, data cleaning were accomplished by using frequency distribution to check for outliers and any erroneous codes.

SPSS analyses were applied to determine whether assumptions of normality, linearity, and homoscedasticity were met for the SSA-R. Diagnostic screening 
procedures revealed that these data met the assumptions of a multiple linear regression. There was a normal distribution of data, and assumptions of linearity and homoscedasticity were met. Further analysis of the data was therefore deemed appropriate (Field, 2009).

\section{Satisfaction with Life Scale (SWLS)}

The five-item Satisfaction with Life Scale (SWLS; Diener et al., 1985) is a self-reported questionnaire that was administered to assess the quality of a participant's life as a whole. The instrument was designed to assess cognitive, rather than affective processes of well-being and life satisfaction. The SWLS was used in the current study to measure adaptation-associated adjustment to college, a QoL criterion variable in the adapted model. The SWLS contains the following items: (a) "In most ways my life is close to the ideal" (b) "The conditions of my life are excellent" (c) "I am satisfied with my life" (d) "So far I have gotten the things I want in life" and, (e) "If I could live my life over, I would change almost nothing." The five items are answered on a 7-point Likert-type scale ranging from 1, 'Strongly disagree" to 7, "Strongly agree."

Corrections for missing data on the SWLS were based on guidelines provided by Tabachnick and Fidell (2012). Although there is no definitive agreement as to an acceptable response rate for the analysis of data for a variable, these authors consider an $80 \%$ response rate acceptable. Therefore, corrections for missing data on the SWLS were made on a one-item basis, with the missing value replaced with the participant's mean score. This procedure eliminated a missing value if no more than 1 item was 
left unanswered on the SWLS. Responses were then summed for an overall or total SWLS score. Higher scores signify higher levels of subjective well-being/life satisfaction. Four cases were deemed to have excessive missing data, and were removed for further consideration of analyses.

Data entry on the SWLS were verified by comparing visually the numbers on a printout of the SPSS data file with the codes on the original web-survey source. After verifying the record, data cleaning were accomplished by using frequency distribution to check for outliers and any erroneous codes.

SPSS analyses were applied to determine whether assumptions of normality, linearity, and homoscedasticity were met. The diagnostic screening procedures revealed that these data met the assumptions of a multiple linear regression. There was a normal distribution of data, and assumptions of normality, linearity, and homoscedasticity were met. Further analyses of data was therefore deemed appropriate (Field, 2009).

\section{Grade Point Average-Scale (GPA-S)}

The Grade Point Average-Scale (GPA-S), adapted by the researcher, is a GPA-derived measure to assess academic performance, a QoL criterion variable in the adapted QoL-PACID model. The GPA-S is located on the Participant Survey. Scores range from 1 (1.66 or below [D average or below]) to 8 (4.00 to 3.67 [A to Aaverage]). A higher numerical score is associated with a higher level of academic performance. Only one score is reported. For example, a respondent with a self-reported 3.20 cumulative GPA will score 6 on the derived measure (i.e., 3.32 to 3.00 [at least a B 
average, but just below a $\mathrm{B}+])$.

In the event of a missing (i.e., unanswered) score, the aggregate mean GPA-S score was used as a replacement. Data entry were verified by comparing visually the number on a printout of the SPSS data file with the codes on the original web-survey source. After verifying the record, data cleaning were accomplished by using frequency distribution to check for outliers or erroneous codes.

SPSS analyses were applied to determine whether assumptions of normality, linearity, and homoscedasticity were met. The diagnostic screening procedures revealed that these data met the assumptions of a multiple linear regression. There was a normal distribution of data, and assumptions of normality, linearity, and homoscedasticity were met. Therefore, further analyses of data was deemed appropriate (Field, 2009).

\section{Student Adaptation to College Questionnaire}

The Student Adaptation to College Questionnaire (SACQ; Baker \& Siryk, 1999) was used to measure psychosocial-emotional adjustment to college, a criterion variable in the adapted QoL-PACID model. The SACQ is a 67-item, self-report questionnaire that yields scores for overall adaptation to college, as well as four facets of college adjustment (i.e., academic, social, personal-emotional, and goal commitmentinstitutional attachment). Each item on the SACQ is rated on a 9-point Likert scale ranging from 1 ("applies very closely to me") to 9 (" doesn't apply to me at all"). The total adjustment score is the sum of the ratings for all 67 items.

Missing data on the SACQ were managed as directed by the scoring and 
procedure manual (Baker \& Siryk, 1999). Corrections for missing data were made on a domain (i.e., four facets of college adjustment: academic, social, personal-emotional, goal commitment/institutional attachment) basis by assigning the value of the average item scores for that domain. This procedure eliminated missing values if no more than 2 items were left unanswered in a domain.

After calculating the raw score from each of the SACQ subscales, they were converted to standardized area $\mathrm{T}$-scores by referring to a table of norms for first-year and second-year college students provided by the instrument developers (Baker \& Siryk, 1999). This transformation allowed the sample scores to be directly interpreted in terms of percentile equivalents of the normative distribution and direct comparisons between domains were possible. The standardized T-score has a mean of 50 and a standard deviation of 10. A range of plus or minus 5 standard deviations is represented by a T-score distribution of $0-100$.

Data entry was verified by comparing visually the numbers printed on a printout of the data file with the numerical information entered on the web survey. After the record was verified, data cleaning was accomplished by using frequency distribution to check for outliers and erroneous codes.

Further analysis was applied to determine whether assumptions of normality and linearity were met for each domain scale (i.e., academic, social, personal-emotional, goal commitment-institutional attachment) of the SACQ. All SACQ domain values were deemed appropriate for further analysis (Field, 2009). However, because of 
highly correlated subscales (inter-correlations ranged from .689 to .859), only the total SACQ score (i.e., Full Scale SACQ Score) was used in further analyses.

\section{Results of Quantitative Analyses}

\section{Descriptive Analysis}

One hundred and three students registered with Offices of Services for Students with Disabilities, and who were attending one of two degree-granting, four-year public postsecondary institutions (both located in the Pacific Northwest) participated in the study. Data were collected during winter, spring, summer, and fall terms, 2008. The analysis of socio-demographic data revealed that respondents ranged in age from 18 to 47 , with a mean age of $22(M=21.91 ; M d n=19 ; S D=6.76)$ years. With respect to gender, $54.8 \%(n=56)$ of respondents were women and $44.2 \%(n=46)$ were men, with $1 \%$ missing data (i.e., non-response) $(n=1)$. These data approximated the distribution of gender in undergraduate students with disabilities at degree-granting, four-year public postsecondary institutions in the United States (56.9\% and 43.1\%, respectively) during the 2007-2008 academic year, as reported by the U.S. Department of Education, National Center for Education Statistics (NCES) (2012).

In the current study, all data were collected online, through a web-based survey. In addition, all potential participants of the target population had equal access to on-campus computers and the internet. The majority of respondents described themselves as White (87.4\%), followed by Asian/Pacific Islander (6.7\%), Black/African American (1.9\%), Native American (1\%), Hispanic (1\%), and Multiple Ethnicities 
(1\%). In addition, there was $1 \%$ missing data (i.e. non-response). This distribution did not reflect that reported by the U.S. Department of Education, NCES (2012) for the 2007-2008 academic year: White (66.3\%), Asian/Pacific Islander (4.8\%), Black/African American (12.6\%), Native American (0.8\%), Hispanic (12.3\%), and other (3.2\%). The current study's sample distribution (in terms of ethnicity) did however, accurately reflect the unequal distribution of private (i.e., individual households) internet and web users in the general U.S. population at the time this survey was conducted (Rainie, 2010). Ying and Newfield note that college students who do not have ready access to a computer in a private (i.e., home) setting may be less apt to respond to and complete a web-based survey. Although conjecture at best, it may have been that the current study's exclusive reliance on online data collection in a more public setting (i.e., college campus) limited the participation of minority students. Nonetheless, generalizability of results, at least in terms of ethnic distribution, must be cautiously interpreted.

Marital status categories included: single (88.5\%), married or partnered (6.7\%), and divorced or separated (3.8\%), with one missing response (1\%). The hours of employment categories for this sample included: none or occasional work hours (64.4\%), eleven to twenty hours (17.3\%), one to ten (10.6), twenty-one to thirty hours (3.8\%), and thirty-one to forty hours (2.9\%). There was also one missing response $(n=1 \%)$. The sample's academic level was reported as $60.6 \%$ first-year $(n=62)$, and $38.4 \%$ second-year $(n=40)$, with one missing response $(n=1 \%)$. 
Data concerning the participants' type of chronic illness and disability included the categories of (a) physical (i.e., sensory or communication disorders [e.g., blindness, deafness], mobility related or orthopedic conditions [e.g., arthritis, spinal cord injury], health and medical conditions [e.g., fibromyalgia, congestive heart failure, multiple sclerosis]; 34.6\%), (b) cognitive/neurocognitive (e.g., attention deficit/hyperactivity disorders, autism spectrum disorders, specific learning disabilities; 45.2\%), and (c) psychiatric (e.g., anxiety disorder, depression, bipolar disorder; 19.2\%). In addition, there was one missing response $(n=1 \%)$. When viewed in their totality, these data suggest that the respondents represented a rather balanced cross-section of postsecondary students with disabilities. Available national distribution figures for type of chronic illness and disability reported by undergraduates attending two-year and four-year degree-granting postsecondary institutions (Raue \& Lewis, 2011) were as follows: physical disabilities (e.g., sensory or communication disorders, mobility or orthopedic, health and medical conditions; 25\%), cognitive/neurocognitive disabilities (e.g., attention deficit/hyperactivity disorders, autism spectrum disorders, specific learning disabilities; 57\%), psychiatric disabilities (e.g., anxiety disorder, depression, bipolar disorder; 16\%), and other disabilities (not specifically reported; 2\%). Although the current sample was slightly over-represented by students with physical disabilities (34.6\% compared to $25 \%$, respectively), slightly under-represented by students with cognitive/neurocognitive disabilities (45.2\% compared to 57\%, respectively), and also slightly over-represented by students with psychiatric disabilities (19.2\% compared to 
$16 \%$, respectively), there were no flagrant deviations (i.e., severe over- or under- representations) in any of the disabling condition categories. Therefore, in terms of disability distribution, the current sample achieved a fairly accurate degree of representativeness, compared to the national U.S. breakdown of disabling conditions among postsecondary students with disabilities attending two-year and four-year public postsecondary institutions (Raue \& Lewis, 2011).

Lastly, disability-related information was obtained regarding age at diagnosis of the disabling condition. These data ranged from birth to 40 years $(M=12.65$; $M D N=12 ; S D=8.01)$, with one missing response $(N=102)$.

\section{Zero-Order Correlations}

Table 1 presents the means and standard deviations for the variables included in the study's correlational (i.e., bivariate and multiple regression) analyses. Prior to addressing the three research aims and one sub-aim, zero-order correlations were calculated among the study's variables. Results of the zero-order correlations between predictor variables and the study's three criterion measures of college adaptation are reported in Table 2.

Among the socio-demographic variables only gender, hours of employment, and age at disability diagnosis, significantly correlated with any of the three adaptation-associated criterion measures (see Table 2). Specifically, gender, a person-related biological status variable, was correlated significantly $(r=.185 ; p<.05)$ with one criterion measure, psychosocial adjustment to college (as measured by the 
Student Adjustment to College Questionnaire [SACQ], Baker \& Siryk, 1999). Hours of employment, a vocation-related variable, was significantly correlated $(r=-.172$; $p<.05$ ) with one criterion measure; satisfaction with life (as measured by the Satisfaction with Life Scale [SWLS]; Diener et al.,1985). Lastly, age at disability diagnosis, a disability-related variable, correlated significantly with all three criterion variables; namely, satisfaction with life (SWLS) $(r=-.189 ; p<.05)$, academic performance (GPA-S) $(r=-.297 ; p<.01)$, and psychosocial-emotional adjustment to college (SACQ) $(r=-.213 ; p<.05)$.

Hypotheses 1 through 4 used bivariate correlation analyses to examine relationships between predictor variables (i.e., college stress; functional limitations; engagement-type coping strategies; perceived social support) and the study's three criterion measures of adjustment to college (i.e., life satisfaction; academic performance; psychosocial adjustment to college).

Hypotheses 5 through 8 utilized hierarchical multiple regression analysis (MRA) to test each moderator (i.e., interaction) model. Consistent with the recommendations of Aiken and West (1991), the predictor variables and the potential moderators were centered prior to the moderation analysis so that each variable had a mean of zero. Thus, the sample mean was subtracted from each individual score, and the interaction variable was created by multiplying the centered predictor by the centered potential moderator variable. Aiken and West (1991) note that centering lessens multicollinearity problems due to scaling in regression equations that contain higher order terms such as 
interactions.

Table 1

Means and Standard Deviations for Predictor and Outcomes

Variables Mean Standard Deviation

\section{Background Characteristics}

Gender

Hours Employed

Age Disability Diagnosed

\section{Stress-Related Predictors}

College-Related Stress (CSI)

Functionality (DFLS)

\section{Resource-Related Predictors}

Engagement Coping (E-COPE)

Disengagement Coping (D-COPE)

Perceived Social Support (SSA-R)

$\begin{array}{rr}.45 & .50 \\ 1.69 & 1.1 \\ 12.65 & 8.0\end{array}$

$40.28 \quad 14.3$

$16.08 \quad 4.6$

$\begin{array}{rr}27.84 & 5.365 \\ 27.25 & 8.531 \\ 75.70 & 12.322\end{array}$

\section{Criterion Adjustment Outcomes}

$\begin{array}{lrr}\text { Satisfaction with Life (SWLS) } & 22.99 & 8.484 \\ \text { Academic Performance (GPA-S) } & 5.59 & 1.611 \\ \text { Psychosocial-Emotional Adjustment (SACQ-T) } & 45.71 & 12.470\end{array}$

Note. CSI = College Stress Inventory; DFLS = Disability Functional Limitations Scale; E-COPE = Engagement Coping; D-COPE = Disengagement Coping; SSA-R = Social Support Appraisals-Revised scale; SWLS = Satisfaction With Life Scale; GPA-S = Grade Point Average-Scale; SACQ-T = Student Adaptation to College Questionnaire-Total score 
Table 2

Zero-Order Correlations for Predictor and Outcome Adjustment Variables

\begin{tabular}{lllllllllll}
\hline Variable & 1 & 2 & 3 & 4 & 5 & 6 & 7 & 8 & 9 & 10 \\
\hline
\end{tabular}

1. Gender

2. Hrs. Emp. $\quad .024$

3. Age. Dis. Dx. $-.230 * * .032$

4. CSI $\quad .102 \quad .213 \quad .214^{*}$

5. DFLS $\quad .082 \quad-.015 \quad .281 * * .582 *$

$\begin{array}{llllll}\text { 6. E-COPE. } & -.142 & .075 & .147 & -.072 & .015\end{array}$

7. D-COPE $\quad-.073 \quad .234 * * .071 \quad .315 * * \quad .178 *-.363 * *$

8. SSA-R $\quad .060 \quad-.080-.259 *-.344 * *-.290 * * .420 * * \quad-.558 * *$

9. SWLS $\quad-.029-.172 *-.189 *-.359 * * \quad-.143 \quad .475 * *-.756 * * \quad .673 * *$

10. GPA-S $\quad-.039-.062 \quad-.297 * *-.206 * \quad-.080 \quad .215^{*} \quad-.492 * * \quad .273 * * \quad .476 * *$

11. SACQ-T $\quad .185 *-.092 \quad-.213 *-.364 * *-.176^{*} \quad .444 * *-.618 * * \quad 668 * * \quad .689 * \quad .363 * *$

$* p<.05 ; * * p<.01$

Note. Hrs. Emp. = Hours Employed; Age Dis. Dx. = Age Disability Diagnosed; CSI = College Stress Inventory; DFLS = Disability Functional Limitations Scale; E-COPE = Engagement Coping; D-COPE = Disengagement Coping; SSA-R = Social Support Appraisal scale-Revised; SWLS = Satisfaction with Life Scale; GPA-S = Grade Point Average Scale; SACQ-T = Student Adaptation to College Questionnaire-Total score.

\section{Testing Bivariate Relationships: Hypotheses 1-4}

Hypothesis 1-A. The first hypothesis, part A stated: There will be a negative relationship between college stress (as measured by the CSI) and global life satisfaction (as measured by the SWLS) in adjustment to college among first-year and second-year students with CID. 
Because the CSI is scored such that the higher the score the higher the level of college stress, and the SWLS is scored such that the higher the score the higher the level of global life satisfaction, a negative relationship between these variables was predicted. The Pearson $r$ correlation revealed a significant inverse relationship between the CSI and the SWLS $(r=-.359, p<.01)$. Therefore, the research hypothesis was supported. Table 3 illustrates how college stress (CSI) was significantly and negatively correlated with adjustment to college, as measured by life satisfaction (SWLS), one of three quality-of-life indicators used in the present study to measure adjustment to college.

Hypothesis 1-B. The first hypothesis, part B stated: There will be a negative relationship between college stress (as measured by the CSI) and academic performance (as measured by the GPA-S]) in first-year and second-year undergraduate students with CID.

Because the CSI is scored such that the higher the score the higher the level of college stress, and the GPA-S is scored such that the higher the score, the higher the level of academic performance, a negative relationship between these variables was predicted. The Pearson $r$ correlation revealed a significant inverse relationship between the CSI and GPA-S $(r=-.206, p<.05)$. Therefore, the research hypothesis was supported. Table 3 illustrates how college stress was significantly and negatively correlated with academic performance, as measured by the GPA-S.

Hypothesis 1-C. The first hypothesis, part C stated: There will be a negative relationship between college stress (as measured by the CSI) and 
psychosocial-emotional college adjustment (as measured by the $S A C Q$ ) among first-year and second-year undergraduate students with CID.

Because the CSI is scored such that the higher the score the higher the level of college stress, and the SACQ is scored such that the higher the score the higher the level of college adjustment, a negative relationship between these variables was predicted. The Pearson $r$ correlation revealed a significant inverse relationship between CSI and SACQ $(r=-.364, p<.01)$. Therefore, the research hypothesis was supported. Table 3 illustrates how college stress (CSI) was significantly and negatively correlated with psychosocial-emotional college adjustment (SACQ) among first-year and second-year undergraduate students with CID.

Table 3

Correlations among Predictor (CSI) and Outcome Measures (SWLS, GPA-S, SACQ-T) in Sample $(N=103)$ of First-Year and Second-Year College Students with CID

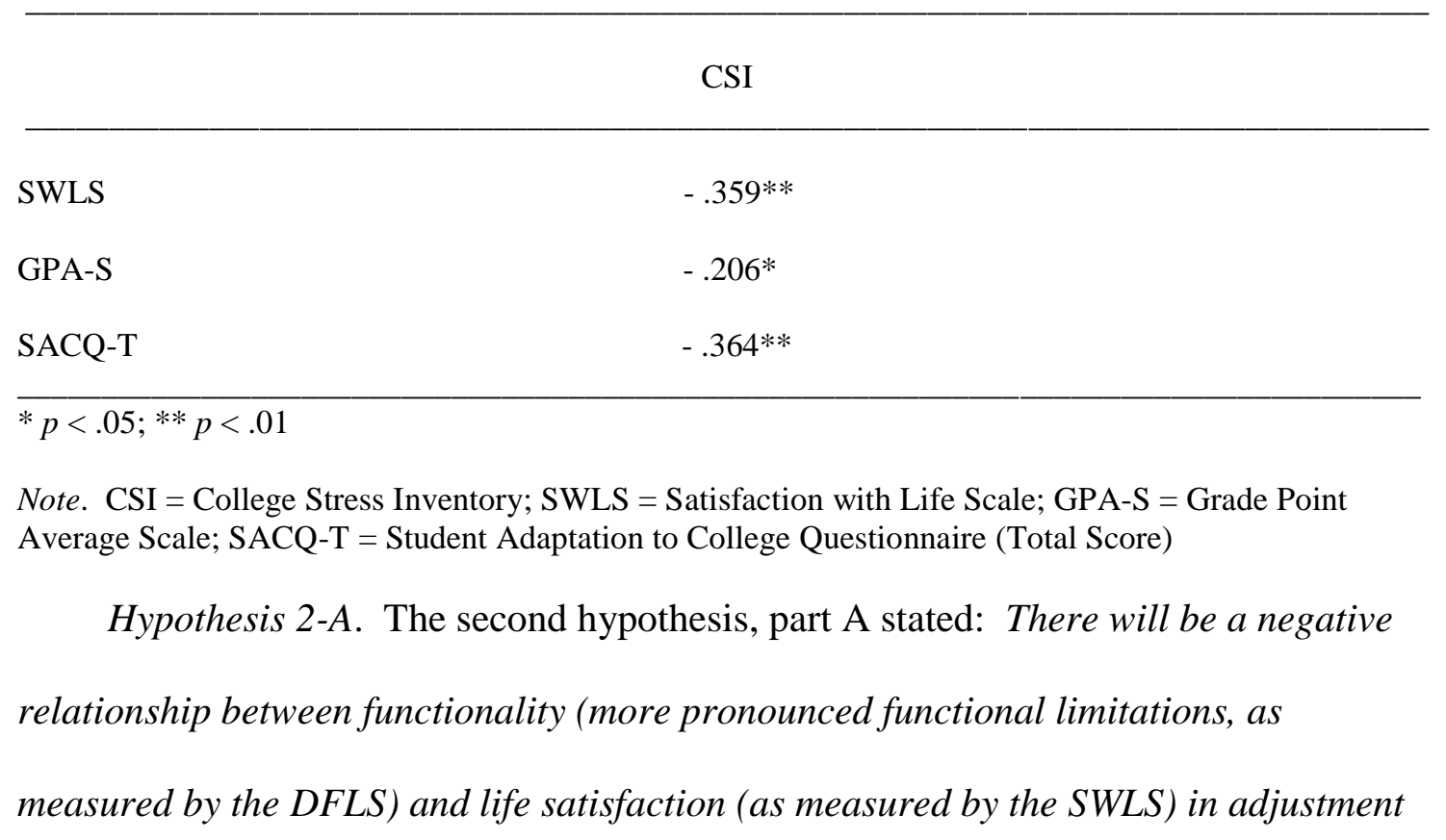


to college among first-year and second-year undergraduate students with CID.

Because the DFLS is scored such that the higher the score the greater the level of functional limitation, and the SWLS is scored such that the higher the score the greater the level of life satisfaction, a negative relationship between these variables was predicted. The total DFLS score was correlated $(r=-.143 ; p>.05)$ with the total SWLS score. The Pearson $r$ correlation however, did not reveal a significant relationship between these variables, although it did show the hypothesized trend. Consequently, the research hypothesis that predicated a negative relationship between functionality (more pronounced functional limitations) and life satisfaction was not supported.

Hypothesis 2-B. The second hypothesis, Part B stated: There will be a negative relationship between functional limitations (i.e., more pronounced functional limitations, as measured by the DFLS) and academic performance (as measured by GPA-S) in first-year and second-year undergraduate students with CID.

Because the DFLS is scored such that the higher the score the greater the level of functional limitations, and the GPA-S is scored such that the higher the score the greater the level of academic success, a negative relationship between these variables was predicted. The total DFLS score was correlated $(r=-.080 ; p>.05)$ with the GPA-S score. The Pearson $r$ correlation revealed no statistically significant relationship between these variables. The second research hypothesis, Part B, was therefore not supported. 
Hypothesis 2-C. The second hypothesis, Part C, stated: There will be a negative relationship between functionality (more pronounced functional limitations, as measured by the DFLS) and psychosocial-emotional adjustment to college (as measured by the $S A C Q$ ) among first-year and second-year undergraduate students with CID.

Because the DFLS is scored such that the higher the score the greater the level of functional limitations, and the SACQ is scored such that the higher the score the greater the level of psychosocial-emotional adjustment to college, a negative relationship between these variables was predicted. The total DFLS score was correlated with the Full Scale (total) score of the SACQ (i.e., SACQ-T). The Pearson $r$ correlation revealed a significant and negative relationship between functional limitations (i.e., more pronounced functional limitations) and the SACQ-T (Total) score $(r=-.176 ; p<.05)$. The second research hypothesis, Part C, was therefore supported. Table 4 illustrates how functional limitations (i.e., more pronounced functional limitations, as measured by the DFLS) was significantly and negatively correlated with the criterion variable, psychosocial-emotional adjustment to college (as measured by the SACQ-T). 
Table 4

Correlations among Predictor (DFLS) and Outcome Measures (SWLS; GPA-S; SACQ-T) in

Sample $(N=103)$ of First-Year and Second-Year College Students with CID

DFLS

$\begin{array}{lc}\text { SWLS } & -.143 \\ \text { GPA-S } & -.080 \\ \text { SACQ-T } & -.176^{*}\end{array}$

$* \mathrm{p}<.05$

Note . DFLS = Disability Functional Limitations Scale; SWLS = Satisfaction with Life Scale;

GPA-S = Grade Point Average-Scale; SACQ-T = Student Adaptation to College Questionnaire-Total score

Hypothesis 3-A. The third hypothesis, part A stated: There will be a positive relationship between engagement coping (as measured by the E-COPE) and global life satisfaction (as measured by the SWLS) in adjustment to college among first-year and second-year undergraduate students with CID.

Because the E-COPE is scored such that the higher the score the greater the level of engagement-type coping, and the SWLS is scored such that the higher the score the greater the level of global life satisfaction, a positive relationship between these variables was predicted. The total E-COPE score, along with the total SWLS score, were correlated. The Pearson $r$ correlation revealed a significant positive relationship between the predictor variable (total E-COPE score), and the criterion variable (SWLS total score $)(r=.475 ; p<.01)$. The research hypothesis was therefore supported. Table 5 illustrates the significant positive correlational relationship between these variables. 
Hypothesis 3-B. The third hypothesis, part B stated: There will be a positive relationship between engagement coping (as measured by the E-COPE) and academic performance (as measured by the GPA-S) in first-year and second-year undergraduate students with CID.

Because the E-COPE is scored such that the higher the score the greater the level of engagement-type coping, and the GPA-S is scored such that the higher the score the greater the level of academic performance, a positive relationship between these variables was predicted. The E-COPE score, along with the GPA-S score, were correlated. The Pearson $r$ correlation revealed a significant positive relationship between the predictor variable (E-COPE), and the criterion variable (GPA-S) $(r=.215$; $p<.05)$. Research hypothesis $3-\mathrm{B}$, was therefore supported. Table 5 illustrate the significant and positive correlation between the two variables.

Hypothesis 3-C. The third hypothesis, part $\mathrm{C}$ stated: There will be a positive relationship between engagement coping (as measured by the E-COPE) and psychosocial-emotional adjustment to college (as measured by the SACQ) among first-year and second-year undergraduate students with CID.

Because the E-COPE is scored such that the higher the score the greater the level of engagement-type coping, and the SACQ is scored such that the higher the score the greater the level of psychosocial-emotional adjustment to college, a positive relationship between these variables was predicted. The total E-COPE score was correlated with the total Full Scale score of the SACQ. The Pearson $r$ correlation 
revealed a significant and positive relationship between predictor variable (i.e., total E-COPE score) and the criterion variable (i.e., total SACQ Full Scale score) $(r=.444$; $p<.01)$. The third research hypothesis, Part C, was therefore supported. Table 5 illustrates how engagement-type coping (E-COPE) was significantly and positively correlated with psychosocial-emotional college adjustment (SACQ-T) among first-year and second-year undergraduate students with CID.

Table 5

Correlations among Predictor (E-COPE) and Outcome Measures(SWLS, GPA-S, SACQ-T) in Sample $(N=103)$ of First Year and Second-Year College Students with CID

\section{E-COPE}

SWLS $.475^{* *}$

GPA-S $.215^{*}$

SACQ-T $\quad .444 * *$

$* \mathrm{p}<.05 ; * * \mathrm{p}<.01$

Note. E-COPE = Engagement Coping; SWLS = Satisfaction with Life Scale; GPA-S = Grade Point Average-Scale; SACQ-T = Student Adaptation to College Questionnaire-Total score

Hypothesis 4-A. The fourth hypothesis, Part A stated: There will be a positive relationship between perceived social support (as measured by the SSA-R) and global life satisfaction (as measured by the SWLS) in adjustment to college among first-year and second-year undergraduate students with CID.

Because the SSA-R is scored such that the higher the score the greater the level of perceived social support, and the SWLS is scored such that the higher the score the greater the level of life satisfaction, a positive relationship between these variables was 
predicted. The total SSA-R score, along with the total SWLS score, were correlated. The Pearson $r$ correlation revealed a significant and positive relationship between the predictor variable (total SSA-R score), and the criterion variable (SWLS total score) $(r=.673 ; p<.01)$. Therefore, research hypothesis 4 -A was supported. Table 6 illustrates the positive correlational relationship between these variables.

Hypothesis 4-B. The fourth hypothesis, part B stated: There will be a positive relationship between perceived social support (as measured by the SSA-R) and academic performance (as measured by the GPA-S) in first-year and second-year undergraduate students with CID.

Because the SSA-R is scored such that the higher the score the greater the level of perceived social support, and the GPA-S is scored such that the higher the score, the greater the level of academic performance, a positive relationship between these variables was predicted. Indeed, the Pearson $r$ correlation revealed a significant and positive relationship between the predictor variable (total SSA-R score), and the criterion variable (GPA-S) $(r=.273 ; p<.01)$. These results therefore support research hypothesis 4-B. Table 6 highlights the positive and correlational relationship between perceived social support (SSA-R) and academic performance, as measured by the GPA-S.

Hypothesis 4-C. The fourth hypothesis, Part C stated: There will be a positive relationship between perceived social support (as measured by the SSA-R) and psychosocial-emotional adjustment to college (as measured by the SACQ) among 
first-year and second-year undergraduate students with CID.

Because the SSA-R is scored such that the higher the score the higher the level of perceived social support, and the SACQ is scored such that the higher the score the higher the level of psychosocial-emotional adjustment to college, a positive relationship between these variables was predicted. The Pearson $r$ correlation revealed a significant and positive relationship between SSA-R and SACQ-T $(r=.668 ; p<.01)$. Therefore, research hypothesis 4-C was supported. Table 6 illustrates how perceived social support (SSA-R) was significantly and positively correlated with psychosocial-emotional adaptation to college (SACQ-T) among first-year and second-year undergraduate students with CID.

Table 6

Correlations among Predictor (SSA-R) and Outcome Measures (SWLS, GPA-S, SACQ-T) in Sample $(N=103)$ of First-Year and Second-Year College Students with CID

SSA-R

$\begin{array}{ll}\text { SWLS } & .673 * * \\ \text { GPA-S } & .273 * * \\ \text { SACQ-T } & .668 * *\end{array}$

$* * p<.01$

Note. SSA-R $=$ Social Support Appraisal-Revised SWLS $=$ Satisfaction with Life Scale; GPA-S $=$ Grade Point Average-Scale; SACQ-T $=$ Student Adaptation to College Questionnaire $($ Total Score)

\section{Testing Moderating (Interactive) Relationships: Hypotheses 5-8}

The second research aim, testing a moderating role for engagement coping and 
perceived social support (each) in stress (i.e., college-related stress; disability-related stress) and college adjustment (as measured by the study's three adaptation-associated criterion variables: satisfaction with life [i.e., SWLS], academic performance [i.e., GPA-S], and psychosocial-emotional adjustment to college [i.e., SACQ-T]) was addressed by performing 12 separate hierarchical multiple regression analyses (MRA's). Each MRA was comprised of 4 Steps. In Step 1, relevant socio-demographic control variables were entered. These included the contribution of one person-related (biological-organismic) variable (i.e., gender), one vocation-related variable (i.e., hours employed), and one disability-related variable (i.e., age disability diagnosed). In Step 2, the unique contribution of each predictor variable: College stress (as measured by the College Stress Inventory (i.e., CSI) and Disability Functional Limitations (as measured by the Disability Functional Limitation Scale (i.e., DFLS) was entered. In Step 3, each potential moderator (i.e., Engagement Coping; Perceived Social Support) was entered. Finally, in Step 4, the specific interaction term (i.e., Engagement Coping X College Stress; Engagement Coping X Functional Limitations; Perceived Social Support X College Stress; Perceived Social Support X Functional Limitations) was entered. Moderation was deemed present when the interaction term was significant (i.e., $<.05)$. An interaction found to be significant was subjected to additional analyses in order to identify the specific conditions under which the moderator affects the relationship between the predictor and the criterion. Tables 7 through 18 present the results of each MRA. 
Hypothesis 5, Part A. The fifth hypothesis, Part A stated: Engagement coping will moderate the impact of college stress on adjustment to college, as measured by satisfaction with life, among first-year and second-year undergraduates with CID.

A hierarchical MRA was conducted using satisfaction with life (i.e., SWLS) as the criterion. In the MRA, the study's three demographic control variables (i.e., gender, age disability diagnosed, hours employed) were entered as Step 1. An examination of Table 7 indicates that these variables combined, were not statistically significant, $R^{2}=.068$, $F(3,99)=2.419, p=.071(p>.05)$. In Step 2, the stress-related predictor variable, college stress (i.e., CSI), was added to the model. The $\Delta \mathrm{R}^{2}$ was statistically significant $\left(\Delta R^{2}=.084\right), \Delta F(1,98)=9.738, p=.002(p<.01)$, indicating that CSI was negatively and significantly predictive of college adjustment, as measured by the SWLS. In Step 3, engagement coping (i.e., E-COPE) as a potential moderator was added to the model. A significant main effect emerged, $\Delta \mathrm{R}^{2}=.238, \Delta \mathrm{F}(1,97)=37.824, \beta=.501(p<.001)$. This finding revealed that engagement coping added significant predictive utility for college adjustment, beyond that measured by the CSI. Moreover, Table 7 shows that engagement coping accounted for approximately $24 \%$ of the variance in adjustment scores. Examination of the $F$ change statistic and weights in Table 7 indicate that engagement coping was the most important predictor in the regression model and was associated with increased adjustment scores. Lastly, in Step 4, the interaction term between engagement coping and college stress (E-COPE X CSI) was added to the model. The $\Delta \mathrm{R}^{2}$ was not statistically significant $\left(\Delta R^{2}=.004, \Delta F(1,96)=.562\right.$, 
$\beta=.062, p=.455(p>.05)$. Examination of the $\mathrm{F}$ change statistic and final weights for the interaction analysis (Table 7) indicates that the interaction of E-COPE and CSI was not a significant overall predictor of college adjustment. Further, the interaction term was not found to significantly increase the amount of variance explained in adjustment when compared to that contributed by the two main predictor variables (i.e., college stress, engagement coping) entered in previous steps.

In summary, results provided support for the direct (main) effect model of engagement coping in the relationship between college-related stress and adjustment to college, as measured by life satisfaction. There was no support for the moderating model of engagement coping in the relationship between college-related stress and life satisfaction in adjustment to college among first-year and second-year undergraduate students with CID. Hypothesis 5-A, therefore, was not supported. 
Table 7

Hierarchical MRA for Moderating Effects of Engagement Coping on the Predictors (College Stress; Engagement Coping) as measured by Life Satisfaction: An Interaction Model

\begin{tabular}{|c|c|c|c|c|c|c|}
\hline $\begin{array}{l}\text { Step \& Predictor } \\
\text { Variables }\end{array}$ & $\mathrm{R}^{2}$ & $\Delta \mathrm{R}^{2}$ & $B$ & $S E B$ & $\beta$ & $\Delta \mathrm{F}$ \\
\hline Step 1 & & & 28.405 & 2.231 & & \\
\hline $\begin{array}{l}\text { Gender, } \\
\text { Age Dis. Dx., } \\
\text { Hours Employed }\end{array}$ & .068 & .068 & -.211 & 1.694 & -.071 & 2.419 \\
\hline Step 2 & & & 26.220 & 2.250 & & \\
\hline CSI & .153 & $.084 * *$ & -.183 & .059 & $-.308 * *$ & $9.738 * *$ \\
\hline Step 3 & & & 27.465 & 1.929 & & \\
\hline E-COPE & .390 & $.238 * * *$ & .792 & .129 & $.501 * * *$ & $37.824 * * *$ \\
\hline Step 4 & & & 27.603 & 1.942 & & \\
\hline $\begin{array}{l}\text { Int. Term: } \\
\text { E-Cope X CSI }\end{array}$ & .394 & .004 & .007 & .009 & .062 & .562 \\
\hline
\end{tabular}

Note. Age Dis. Dx. = Age Disability Diagnosed; CSI = College Stress Inventory (Total Score);

E-Cope = Engagement Coping scale of the Brief COPE; Int. Term = Interaction Term (Engagement Coping [E-COPE] multiplied by College Stress [CSI]).

Hypothesis 5, Part B. The fifth hypothesis, Part B stated: Engagement coping (i.e., E-COPE) will moderate the impact of college-related stress (i.e., College Stress Inventory [CSI]) on academic performance (as measured by the GPA-S), in undergraduate students with CID.

A hierarchical MRA was conducted to address the hypothesis, using the GPA-S as the criterion. Only one demographic control variable was included in the model, age 
disability diagnosed, a disability-related variable. In Step 1, the control variable (i.e., age disability diagnosed) was entered. This variable was statistically significant, $R^{2}=.088, F(1,101)=9.750, p=.002$. In Step 2, the college stress-related variable as measured by the CSI was added to the model, but was not statistically significant as a predictor of academic performance (i.e., GPA-S) $\left(\Delta R^{2}=.021, \Delta F[1,100]=2.398\right.$, $\beta=-.150, p=.125)$. In Step 3 , the predictive role of E-COPE was examined next. The $F$ change statistic and beta weight revealed the emergence of a significant main effect when E-COPE was added to the model $\left(\Delta R^{2}=.061, \Delta F[1,99]=7.293, \beta=.251\right.$, $p=.008[p<.01])$. Further, Table 8 indicates that E-COPE contributed approximately $6 \%$ of the variance in GPA-S scores. Engagement coping was the most important predictor in the regression model, and was associated with more successful academic performance, as measured by the GPA-S. In Step 4, the interaction term between E-COPE and CSI (i.e., E-COPE X CSI) was added to the model. An inspection of the $F$ change statistic and weights reported in Table 8 revealed no detection of a moderator effect $\left(\Delta R^{2}=.000, \Delta F[1,98]=.018, \beta=.012, p=.893\right)$. Evidence that the relationship between engagement coping and college stress conforms to that predicted by a stress-interacting perspective was not supported.

Findings in Table 8 provide empirical support for the direct (main) effect model of engagement coping in the relationship between college-stress and academic performance in first-year and second-year undergraduate students with CID. However, there was no support for the stress-moderating model of engagement-type coping efforts 
in the relation between college stress and academic performance, as measured by the GPA-S. Hypothesis 5-B, therefore, was not supported.

Table 8

Hierarchical MRA for Moderating Effects of Engagement Coping on the Predictors (College Stress; Engagement Coping) as measured by Academic Performance: An Interaction Model

$\begin{array}{lllllll}\text { Step \& Predictor } & \mathrm{R}^{2} & \Delta \mathrm{R}^{2} & B & \mathrm{SEB} & \beta & \Delta \mathrm{F} \\ \text { Variables }\end{array}$

Step 1

Age Dis. Dx.
$.088^{*}$
6.347

.088
.286

.019

$-.297^{*}$

$9.750^{*}$

Step 2

CSI

.109

.021

6.266

.289

$-.017$

.011

$-.150$

2.398
Step 3

E-COPE

$.170 * *$

$.061 * *$

.076

6.374

6.375

.289

Step 4
.000

.171

.000

Int. Term:

E-COPE X CSI

${ }^{*} p<.05 ; * * p<.01$

Note. Age Dis. Dx. = Age Disability Diagnosed CSI = College Stress Inventory; E-COPE $=$ Engagement Coping; Int. Term: = Interaction Term: E-COPE (Engagement Coping) multiplied by CSI (College Stress Inventory)

Hypothesis 5, Part C. The fifth hypothesis, Part C stated: Engagement coping will moderate the impact of college-related stress (i.e., College Stress Inventory [CSI]) on psychosocial-emotional adjustment to college (as measured by the Student Adjustment to College Questionnaire [SACQ]), among first-year and second-year 
undergraduate students with CID.

A hierarchical MRA was conducted to address the hypothesis, using psychosocial adjustment to college (i.e., SACQ-T, Total score) as the criterion. Socio-demographic control variables included gender, a biological status variable, and age disability diagnosed, a disability-related control variable. In Step 1, both control variables (i.e., gender; age disability diagnosed) were entered into the model. This set of demographic variables were statistically significant, $R^{2}=.065, \Delta F(2,100)=3.462$, $p=.035$. In Step 2, the college-related stress variable (as measured by the total CSI score) was added to the model. The CSI was statistically significant as a predictor of psychosocial adjustment to college $\left(\Delta R^{2}=.124, \Delta \mathrm{F}[1,99]=15.173[p<.001]\right)$. In Step 3 , the engagement coping (i.e., E-COPE) predictor was added to the model and a significant main effect emerged, $\Delta R^{2}=.220, \Delta F(1,98)=36.503, \beta=.479(p<.001)$. This finding reveals that engagement coping added significant predictive utility for psychosocial-emotional adjustment to college, beyond the contribution measured by the CSI. Furthermore, examination of Table 9 reveals that E-COPE accounted for approximately $22 \%$ of the variance in college adjustment (i.e., SACQ-T) scores. As indicated in Table 9, the $F$ change statistic and weights show that engagement coping was the most important predictor in the regression model, significantly and positively contributing to psychosocial-emotional adjustment to college. Lastly, in Step 4, the interaction term between engagement coping and college stress (E-COPE X CSI) was added to the model. Table 9 reveals that $\Delta R^{2}$ was not statistically significant 
$\left(\Delta R^{2}=.000, \Delta \mathrm{F}[1,97]=.006, \beta=.006[\mathrm{p}>.05]\right)$. Examination of the $F$ change statistic and final weights for the interaction analysis (Table 9) reveal that the interaction of E-COPE and CSI was not a significant overall predictor of college adjustment. Moreover, the interaction term (E-COPE X CSI) was not found to significantly increase the amount of variance explained in SACQ-T scores, when compared to that contributed by the two main predictor variables (i.e., CSI; E-COPE) entered in Step 2 and Step 3, respectively.

In summary, the results provide support for the direct (main) effect model of engagement coping in the relation between college-related stress and psychosocial-emotional adjustment to college, as measured by the SACQ-T. Analyses offered no support for the moderating (i.e., interacting) model of engagement coping in the relationship between college-related stress and psychosocial adjustment to college among first-year and second-year undergraduate students with CID. Therefore, hypothesis 5-C was not supported. 
Table 9

Hierarchical MRA for Moderating Effects of Coping on the Predictors (College Stress; Engagement Coping) as Measured by Psychosocial-Emotional Adjustment to College: An Interaction Model

\begin{tabular}{|c|c|c|c|c|c|c|}
\hline $\begin{array}{l}\text { Step \& Predictor } \\
\text { Variables }\end{array}$ & $R^{2}$ & $\Delta R^{2}$ & $B$ & $S E B$ & $\beta$ & $\Delta F$ \\
\hline Step 1 & $.065^{*}$ & & 47.652 & 2.738 & & \\
\hline $\begin{array}{l}\text { Gender } \\
\text { Age Dis. Dx. }\end{array}$ & & & & & & \\
\hline Step 2 & & & 45.198 & 2.638 & & \\
\hline CSI & $.189 * * *$ & $.124 * * *$ & -.319 & .082 & $-.366 * * *$ & $15.173 * * *$ \\
\hline Step 3 & & & 46.027 & 2.268 & & \\
\hline E-COPE & $.409 * * *$ & $.220 * * *$ & 1.114 & .184 & $.479 * * *$ & $36.503 * * *$ \\
\hline Step 4 & & & 46.047 & 2.295 & & \\
\hline $\begin{array}{l}\text { Int. Term: } \\
\text { E-COPE X CSI }\end{array}$ & .409 & .000 & .001 & .013 & .006 & .006 \\
\hline
\end{tabular}

$* p<.05 ; * * * p<.001$

Note. Age Dis. Dx. = Age Disability Diagnosed; CSI = College Stress Inventory; E-COPE = Engagement Coping; Int. Term = Interaction Term: E-COPE X CSI = Engagement Coping multiplied by College Stress Inventory.

Hypothesis 6, Part A. The sixth hypothesis, Part A stated: Engagement coping will moderate the impact of disability-related stress (i.e., Disability Functional Limitations Scale [DFLS]) on adjustment to college, as measured by satisfaction with life (i.e., SWLS) among first-year and second-year undergraduates with CID.

A hierarchical MRA was conducted using satisfaction with life (as measured by the SWLS) as the criterion. In the MRA, the control variables (i.e., gender, age disability diagnosis, hours employed) were entered as Step 1. These variables 
combined, were not statistically significant, $R^{2}=.068, \Delta F(3,99)=2.42, p=.071$. In Step 2, the predictor, disability functional limitations (DFLS), was added to the model. The $\Delta R^{2}$ was not statistically significant $\left(\Delta R^{2}=.008, F[1,98]=.822, p=.37\right)$. In Step 3 , the engagement coping predictor was added to the model, whereby a significant main effect emerged, $\Delta R^{2}=.266, \Delta F(1,97)=39.25, p<.001$. This finding suggests that engagement coping adds significant predictive utility for college adjustment (as measured by satisfaction with life), over and above disability functional limitations (DFLS). Moreover, Table 10 shows that engagement coping accounted for approximately $27 \%$ of the variance in adjustment scores. Finally, in Step 4, the interaction term engagement coping and functional limitations (i.e., E-COPE X DFLS) was added to the regression model. The $\Delta R^{2}$ was statically significant $\left(\Delta R^{2}=.048\right.$, $\Delta F[1,96]=7.52, p<.001)$, suggesting that together, this interaction added significant predictive utility for adjustment to college (as measured by life satisfaction). Table 10 reveals the interaction term (i.e., E-COPE X DFLS) as significant, after controlling for the main effects $(\beta=.029, p<.01)$. Engagement coping thus met the criteria for an interacting variable between DFLS and SWLS scores.

Additional analysis was conducted for this possible moderator as recommended by Aiken and West (1991) and Holmbeck (2002). High and low variables for E-COPE and DFLS were computed by adding and subtracting one standard deviation from the centered value for each participant. These new variables (high/low engagement coping) and (high/low functional limitations) were entered into separate simultaneous 
regressions. Their regression lines were graphed based on the equations from the separate model including the simple slope for the moderator variable (i.e., E-COPE) and y-intercept (i.e., life satisfaction; SWLS) (see Figure 1). This additional analysis supported the significant interacting effect for engagement coping on the relationship between disability-related functional limitations (i.e., DFLS) and adjustment to college, as measured by life satisfaction (i.e., SWLS). As illustrated in Figure 1, the relationship between functional limitation (i.e., DFLS) severity and adjustment (as measured by life satisfaction (i.e., SWLS) is stronger for those individuals who perceive higher, rather than lower, engagement coping (i.e., E-COPE) styles.
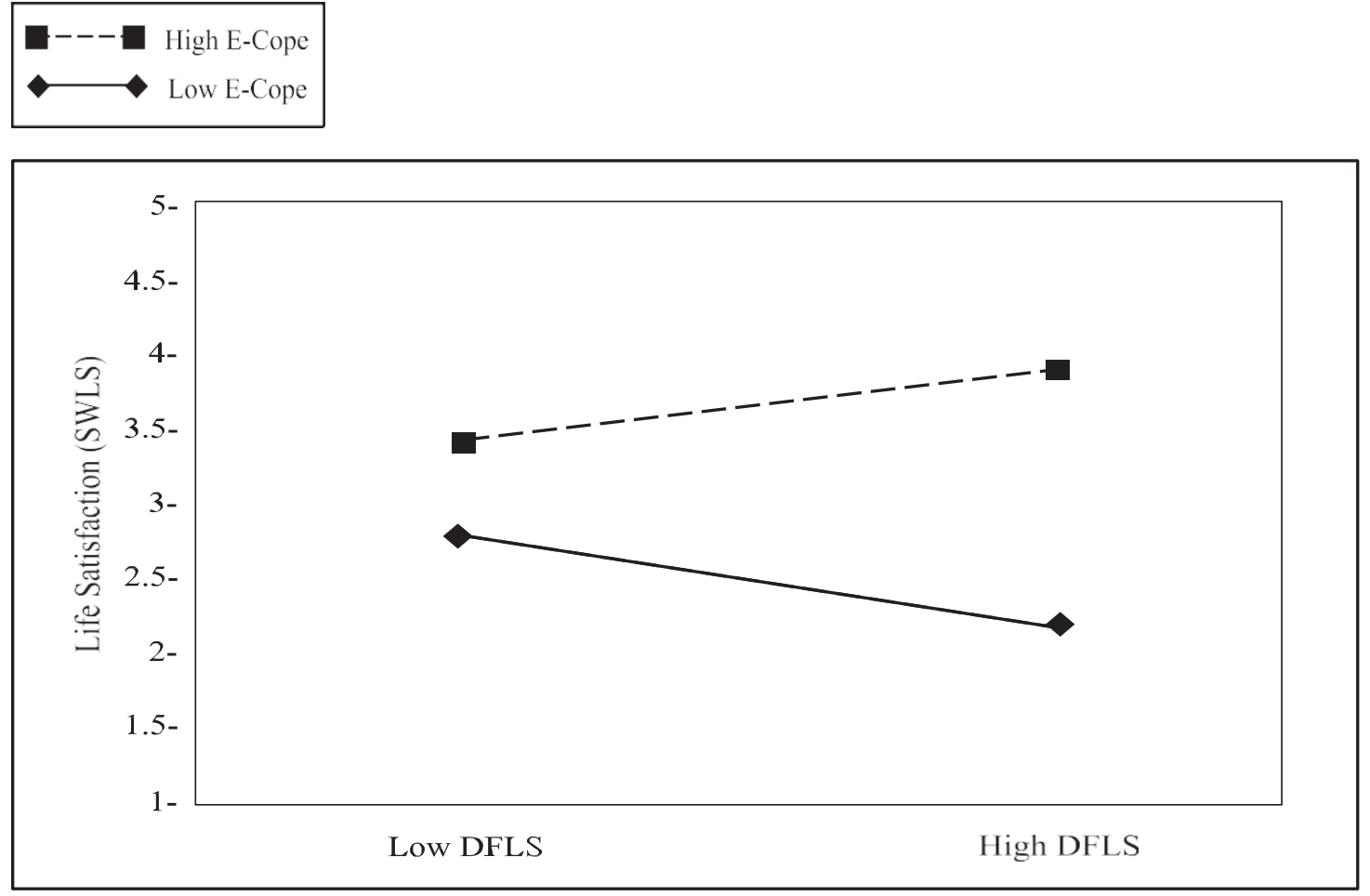

Figure 1. Interaction Effects of DFLS and E-COPE on Life Satisfaction (SWLS) 
The nature of this significant interaction suggests that high engagement coping (i.e., high E-COPE) affects life satisfaction (i.e., as measured by the SWLS) significantly more than low engagement coping (i.e., low E-COPE), but only for those respondents endorsing high disability functional limitation scores (i.e., high DFLS, as measured by increased restrictions in the ability to perform daily activities and life roles). At low DFLS, high E-COPE is only slightly better than low E-COPE on life satisfaction scores. At high DFLS however, high E-COPE is "significantly" better than low E-COPE on life satisfaction scores (i.e., as measured by the SWLS). Engagement coping, therefore, played a moderating (i.e., interacting) role between functionality and college adjustment (as measured by life satisfaction) in this sample of first-year and second-year undergraduate college students with CID adjusting to college. Thus, hypothesis 6-A was supported. 
Table 10

Hierarchical MRA for Moderating Effects of Coping on the Predictors (Functional Limitations; Engagement Coping) as Measured by Life Satisfaction: An Interaction Model

\begin{tabular}{|c|c|c|c|c|c|c|}
\hline $\begin{array}{l}\text { Step \& Predictor } \\
\text { Variable }\end{array}$ & $\mathrm{R}^{2}$ & $\Delta \mathrm{R}^{2}$ & $B$ & $S E B$ & $\beta$ & $\Delta \mathrm{F}$ \\
\hline Step 1 & .068 & .068 & 28.41 & 2.23 & -.071 & 2.42 \\
\hline $\begin{array}{l}\text { Gender } \\
\text { Age Dis. Dx. } \\
\text { Hrs. Employed }\end{array}$ & & & $\begin{array}{l}-1.21 \\
-.21 \\
-.16\end{array}$ & $\begin{array}{c}1.70 \\
.106\end{array}$ & $\begin{array}{l}-.071 \\
-.201 * *\end{array}$ & \\
\hline Step 2 & & & 27.94 & 2.30 & & .822 \\
\hline DFLS & .076 & .008 & -.17 & & -.093 & \\
\hline Step 3 & & & 28.84 & 1.95 & & \\
\hline E-COPE & $.342 * * *$ & $.266^{* * *}$ & .83 & .13 & $.526 * * *$ & $39.25^{* * * *}$ \\
\hline Step 4 & & & 28.93 & 1.89 & & \\
\hline $\begin{array}{l}\text { Int. Term: } \\
\text { E-COPE X } \\
\text { DFLS }\end{array}$ & $.390 * *$ & $.048 * *$ & $.079 * *$ & .029 & $.029 * *$ & $.229 * *$ \\
\hline
\end{tabular}

$* * p<.01 ; * * * p<.001$

Note. Age Dis. Dx.. = Age Disability Diagnosed; Hrs Employed = Hours of Employment; DFLS = Disability Functional-Limitations Scale; E-COPE = Engagement Coping; Int. Term = Interaction Term: E-COPE X DFLS = Engagement Coping X Disability Functional Limitations Scale

Hypothesis 6, Part B. Hypothesis six, Part B stated: Engagement coping will moderate the impact of functional limitations (i.e., increased functional limitations) on academic performance (as measured by GPA-S), in first-year and second-year undergraduates with CID.

A hierarchical MRA was conducted using academic performance (i.e., GPA-S) as the criterion variable. Only one socio-demographic control variable was included in the 
analysis: age disability diagnosed, a disability-related variable. In Step 1, the the socio-demographic disability-related control variable, age disability diagnosed, was first entered, and was statistically significant, $R^{2}=.088, F(1,101)=9.75, p=<.01$. In Step 2, the predictor variable, functional limitations (i.e., DFLS) was added to the model. The $\Delta R^{2}$ was not statistically significant, $\Delta F(1,100)=.002, p>.05$. In Step 3, the engagement coping predictor was added to the model, whereby a significant main effect emerged. Specifically, the $\Delta R^{2}$ was statistically significant, $\Delta F(1,99)=8.04$, $p<.01$. This finding suggests that engagement coping adds significant predictive utility for academic performance, as measured by GPA-S. Moreover, Table 11 shows that E-COPE accounted for approximately $7 \%$ of the variance in academic performance scores. Finally, in Step 4, the interaction term between engagement coping and functional limitations (i.e., E-COPE X DFLS) was added to the regression model. The $\Delta R^{2}$ was not statistically significant, $\Delta F(1,98)=.288, p>.05$. Further, the interaction term was not found to significantly increase the amount of variance explained in GPA-S scores, when compared to that contributed by the predictor E-COPE, entered in the previous step. Evidence that the relationship between engagement coping and functional limitations conforms to that predicted by a stress-interacting perspective was not statistically supported in the model.

Findings for hypothesis six, Part B, provide support for the direct (main) effect model of engagement coping strategies in the relationship between disability-related functional limitations (i.e., DFLS) and academic performance (i.e., as measured by 
GPA-S) among first-year and second-year undergraduate students with CID.

However, there was no support for the moderating (i.e., interacting) model of engagement-type coping strategies in the relationship between functional limitations (i.e., as measured by an increased level of functional limitations), a disability-related variable, and academic performance (i.e., as measured by the GPA-S), an adaptation-associated criterion variable. Therefore, hypothesis 6-B was not supported.

Table 11

Hierarchical MRA for Moderating Effects of Coping on the Predictors (Functional Limitations; Engagement Coping) as Measured by Academic Performance: An Interaction Model

\begin{tabular}{|c|c|c|c|c|c|c|}
\hline $\begin{array}{l}\text { Step \& Predictor } \\
\text { Variable }\end{array}$ & $R^{2}$ & $\Delta R^{2}$ & $B$ & $S E B$ & $\beta$ & $\Delta F$ \\
\hline Step 1 & & & 6.347 & .286 & & \\
\hline Age Dis. Dx. & .088 & $.088^{*}$ & -.060 & .019 & $-.297 * *$ & $9.75 * *$ \\
\hline Step 2 & & & 6.350 & .296 & & \\
\hline DFLS & .088 & .000 & .001 & .035 & .004 & .002 \\
\hline Step 3 & & & 6.454 & .288 & & \\
\hline E-COPE & .157 & $.068 * *$ & .080 & .028 & $.265^{* *}$ & $8.038 * *$ \\
\hline Step 4 & & & 6.463 & .290 & & \\
\hline $\begin{array}{l}\text { Int. Term: } \\
\text { E-COPE X DFLS }\end{array}$ & .159 & .002 & -.003 & .006 & -.051 & .288 \\
\hline
\end{tabular}

Note. Age Dis. Dx. = Age Disability Diagnosed; DFLS $=$ Disability Functional Limitation Scale; E-COPE = Engagement Coping; Inter. Term = Interaction Term: E-COPE X DFLS = Engagement Coping multiplied by Disability Functional Limitation Scale

Hypothesis 6-C. Hypothesis six, Part C stated: Engagement coping will moderate 
the impact of disability functional limitations (as measured by the Disability Functional Limitations Scale [DFLS, Total scale]) on psychosocial-emotional adjustment to college (as measured by the Student Adjustment to College Questionnaire [SACQ, Full Scale score] among first-year and second-year undergraduates with CID).

A hierarchical MRA was conducted to address the hypothesis, using the Full-Scale (i.e., total) SACQ score (i.e., SACQ-T) as the criterion. Socio-demographic control variables included gender, a person-related variable, and age disability diagnosed, a disability-related variable. In Step 1, the control variables (i.e., gender, age disability diagnosed) were first entered. These variables combined, were statistically significant in the prediction of SACQ-T college adjustment scores, $R^{2}=.065$, $F(2,100)=3.462, p=.035$. In Step 2, the disability-related predictor variable, DFLS, was added to the model, but failed to reach statistical significance (i.e., $\Delta R^{2}=.021$, $\Delta F(1,99)=2.287, \beta=-.153, p=.134$. In Step 3, the predictor measuring engagement-type coping efforts (E-COPE) was added to the model, and a significant main effect emerged $\left(\Delta R^{2}=.248, \Delta F[1,98]=36.493, \beta=.507, p<.001\right)$. The $\Delta F$ statistic and $\beta$ weight in Table 12 reveal that E-COPE adds significant predictive utility for SACQ scores. Further, E-COPE accounted for approximately $25 \%$ of the variance in psychosocial-emotional adjustment to college scores (i.e., SACQ-T). Engagement-type coping was the most important predictor in the regression model and was associated with increased psychosocial-emotional adjustment to college scores. In Step 4, the interaction term (E-COPE X DFLS) was added to the model, but was not a significant 
predictor of college adjustment, as measured by psychosocial-emotional adjustment to college scores (i.e., SACQ-T). Specifically, the $\Delta F$ statistic and $\beta$ weight for the interaction analysis (Table 12) indicates that the interaction of E-COPE with DFLS was not a significant overall predictor of SACQ-T scores (i.e., $\Delta R^{2}=.000$, $\Delta F[1,97]=.030, \beta=.015, p=.862)$. Moreover, the interaction term (E-COPE X DFLS) was not found to significantly increase the amount of variance explained in psychosocial-emotional adjustment to college, when compared to that contributed by the engagement coping variable, entered in the previous step (Step 3).

The results for hypothesis 6-C provide support for the direct (main) effect model of engagement coping in the relationship between functional limitations and psychosocial-emotional adjustment to college among first-year and second-year undergraduate students with CID. There was no support however, for the moderating (i.e., interacting) model of engagement coping (i.e., E-COPE) in the relationship between disability-related functional limitations (i.e., DFLS), and psychosocial-emotional adjustment to college (i.e., SACQ-T [total score]). Therefore, hypothesis 6-C was not supported. 
Table 12

Hierarchical MRA for Moderating Effects of Coping on the Predictors (Functional Limitations; Engagement Coping) as Measured by Psychosocial-Emotional Adjustment to College: An Interaction Model

\begin{tabular}{|c|c|c|c|c|c|c|}
\hline $\begin{array}{l}\text { Step \& Predictor } \\
\text { Variables }\end{array}$ & $R^{2}$ & $\Delta R^{2}$ & $B$ & $S E B$ & $\beta$ & $\Delta F$ \\
\hline Step 1 & $.065^{*}$ & & 47.652 & 2.738 & & \\
\hline Gender & & & 3.579 & 2.481 & .143 & \\
\hline Age Dis. Dx. & & & -.280 & .155 & -.180 & \\
\hline Step 2 & & & 46.431 & 2.838 & & \\
\hline DFLS & .086 & .021 & -.412 & .272 & -.153 & 2.287 \\
\hline Step 3 & & & 47.030 & 2.437 & & \\
\hline E-COPE & $.334 * * *$ & $.248 * * *$ & 1.178 & .195 & $.507 * * *$ & $36.493 * * *$ \\
\hline $\begin{array}{l}\text { Step } 4 \\
\text { Inter. Term: } \\
\text { E-COPE X DFLS }\end{array}$ & .334 & .000 & $\begin{array}{r}47.064 \\
.008\end{array}$ & $\begin{array}{r}2.456 \\
.044\end{array}$ & .015 & .030 \\
\hline \multicolumn{7}{|c|}{$* \mathrm{p}<.05 ; * * * \mathrm{p}<.001$} \\
\hline \multicolumn{7}{|c|}{$\begin{array}{l}\text { Note. Age Dis. Dx. = Age Disability Diagnosed; DFLS = Disability Functional Limitation Scale; } \\
\text { E-COPE = Engagement Coping; Inter. Term = Interaction Term: E-COPE X DFLS = Engagement } \\
\text { Coping multiplied by Disability Functional Limitation Scale. }\end{array}$} \\
\hline \multicolumn{7}{|c|}{ Hypothesis 7-A. Hypothesis seven, Part A stated: Perceived social support will } \\
\hline \multicolumn{7}{|c|}{ moderate the impact of college stress (as measured by the College Stress Inventory } \\
\hline \multicolumn{7}{|c|}{ [CSI]) on psychosocial adjustment to college (as measured by life satisfaction[SWLS] } \\
\hline \multicolumn{7}{|c|}{ among first-year and second-year undergraduate students with CIDs. } \\
\hline \multicolumn{7}{|c|}{ A hierarchical MRA was conducted to address the hypothesis using life } \\
\hline \multicolumn{7}{|c|}{ satisfaction (as measured by the SWLS) as the criterion. Socio-demographic control } \\
\hline
\end{tabular}


vocational-related variable, and age disability diagnosed, a disability-related variable. In Step 1, the control variables (i.e., gender, hours employed, age disability diagnosed) were first entered. The combined three control variables failed to contribute significantly to the prediction of SWLS scores (i.e., $R^{2}=.068, \Delta F[3,99]=2.42$, $p>.05]$ ). In Step 2, the college-related predictor (i.e., college stress, as measured by the CSI) variable was added to the model and was statistically significant, $\Delta R^{2}=.084$, $\Delta F(1,98)=9.738, p=.002(p<.01)$. This finding suggests that college stress (i.e., as measured by the CSI) adds significantly to the prediction of adaptation to college, as measured by life satisfaction. Additionally, college stress accounted for approximately $8 \%$ of the variance in life satisfaction scores. In Step 3, the potential moderator, perceived social support (as measured by the SSA-R), was added to the model. As indicated in Table 13, the $\Delta R^{2}$ was statistically significant $\left(\Delta R^{2}=.330\right.$, $\Delta F[1,97]=61.938,[p<.001])$. This finding suggests that perceived social support adds significant predictive utility for college adjustment, as measured by life satisfaction (i.e., SWLS). Moreover, Table 13 shows that perceived social support accounted for approximately $33 \%$ of the variance in adjustment scores. Examination of the $F$ change statistic and weights in Table 13 indicate that perceived social support was the most important predictor in the regression model, and was associated with increased life satisfaction scores. Finally, in Step 4, the interaction term between perceived social support and college stress (SSA-R X CSI) was added to the model. The $\Delta R^{2}$ was not statistically significant $\left(\Delta R^{2}=.011, \Delta F[1,96]=2.144, \beta=.114, p=.146[p>.05]\right)$. 
Examination of the $F$ change statistic and weights for the interaction analysis (Table 13) indicates that the interaction (SSA-R X CSI) was not a significant overall predictor of college adjustment, as measured by life satisfaction scores. Further, the interaction term was not found to significantly increase the amount of variance explained in college adjustment when compared to that contributed by the two main predictor variables (i.e., college stress, perceived social support) entered in previous steps.

Findings provide support for the direct (main) effect model of perceived social support in the relationship between college stress and psychosocial adaptation to college (as measured by life satisfaction) among first-year and second-year undergraduate students with CID. There was no support however, for the moderator (interaction) model of perceived social support in the relationship between college stress, a collegerelated variable (as measured by the CSI) and adaptation to college (as measured by the SWLS). Put differently, there was no evidence that perceived social support either attenuated the strength of the relationship between college stress and adjustment to college, or affected adjustment in interaction with college stress. Therefore, hypothesis 7-A was not supported. 
Table 13

Hierarchical MRA for Moderating Effects of Perceived Social Support on the Predictors (College Stress; Perceived Social Support) as Measured by Life Satisfaction: An Interaction Model

\begin{tabular}{|c|c|c|c|c|c|c|}
\hline $\begin{array}{l}\text { Step \& Predictor } \\
\text { Variables }\end{array}$ & $\mathrm{R}^{2}$ & $\Delta \mathrm{R}^{2}$ & $B$ & $S E B$ & $\beta$ & $\Delta \mathrm{F}$ \\
\hline Step 1 & .068 & .068 & 28.405 & 2.231 & & 2.419 \\
\hline \multicolumn{7}{|l|}{$\begin{array}{l}\text { Gender, } \\
\text { Hrs. employed, } \\
\text { Age Dis. Dx. }\end{array}$} \\
\hline Step 2 & & & 26.220 & 2.250 & & \\
\hline CSI & .153 & $.084 * *$ & -.183 & .059 & -.308 & $9.738 * *$ \\
\hline Step 3 & & & 24.858 & 1.775 & & \\
\hline SSA-R & .483 & $.330 * * *$ & .431 & .055 & .626 & $61.938 * * *$ \\
\hline Step 4 & & & 25.243 & 1.784 & & \\
\hline $\begin{array}{l}\text { Int. Term: } \\
\text { SSA-R X CSI }\end{array}$ & .494 & .011 & .006 & .004 & .114 & 2.144 \\
\hline
\end{tabular}

$* * \mathrm{p}<.01 ; * * * \mathrm{p}<.001$

Note. Hrs. employed = Hours employed; Age Dis. Dx. = Age Disability Diagnosed CSI = College Stress Inventory; SSA-R. = Social Support Appraisal-Revised scale; Int. Term: = Interaction Term (Perceived Social Support X College Stress).

Hypothesis 7-Part B. The seventh hypothesis, Part B stated: Perceived Social Support will moderate the impact of college stress on academic performance (as measured by GPA-S) in first-year and second-year undergraduate with CIDs.

A hierarchical MRA was conducted to address the hypothesis using GPA-S as the criterion. Only one socio-demographic control variable was included in the model: age disability diagnosed, a disability-related variable. In Step 1 the control variable, age 
disability diagnosed, was first entered. This socio-demographic control variable contributed significantly to the prediction of GPA-S adjustment scores, $R^{2}=.088$, $\Delta F(1,101) 9.750, p=.002(p<.01)$. In Step 2, the college-related predictor variable (as measured by the CSI) was added to the model, $\Delta R^{2}=.021, \Delta F=2.398(p>.05)$, and was not statistically significant. As a predictor variable, college stress did not add any unique contribution to the model. In Step 3, the role of perceived social support (i.e., SSA-R) as a potential predictor of academic performance (i.e., GPA-S) was added to the regression model. As indicated in Table 14, the SSA-R variable did not add any significant predictive utility to the regression model $\left(\Delta R^{2}=.028, \Delta F[1,99]=3.181\right.$, $p>.05$ ). The direct (main) effect of perceived social support in the relationship between college stress and academic performance, therefore, was not supported. Finally, in Step 4, the interaction term between perceived social support and college stress (i.e., SSA-R X CSI) was added to the model. Examination of the $F$ change statistic and weights (Table 14) indicate that the interaction of perceived social support with college stress was not a significant overall predictor of academic performance, as measured by GPA-S (i.e., $\left[\Delta \mathrm{R}^{2}=.002, \Delta \mathrm{F}[1,98]=.187[p>.05]\right)$.

In summary, the results (Table 14) do not provide support for either a direct (main) effect model or moderator model of perceived social support in the relationship between college stress and academic performance in first-year and second-year undergraduate students with CID. Therefore, hypothesis 7-B was not supported. 
Table 14

Hierarchical MRA for Moderating Effects of Perceived Social Support on the Predictors (College Stress; Perceived Social Support) as Measured by Academic Performance: An Interaction Model

\begin{tabular}{|c|c|c|c|c|c|c|}
\hline $\begin{array}{l}\text { Step \& Predictor } \\
\text { Variables }\end{array}$ & $\mathrm{R}^{2}$ & $\Delta \mathrm{R}^{2}$ & $B$ & $S E B$ & $\beta$ & $\Delta \mathrm{F}$ \\
\hline Step 1 & & & 6.347 & .286 & & \\
\hline Age Dis. Dx. & .088 & $.088 * *$ & -.060 & .019 & $-.297 * *$ & $9.750 * *$ \\
\hline Step 2 & & & 6.266 & .289 & & \\
\hline CSI & .109 & .021 & -.017 & .011 & -.150 & 2.398 \\
\hline Step 3 & & & 6.176 & .290 & & \\
\hline SSA-R & .137 & .028 & .024 & .013 & .181 & 3.181 \\
\hline Step 4 & & & 6.165 & .292 & & \\
\hline $\begin{array}{l}\text { Int. Term: } \\
\text { SSA-R X CSI }\end{array}$ & .139 & .002 & .000 & .001 & -.043 & .187 \\
\hline
\end{tabular}

$* * \mathrm{p}<.01$

Note. Age Dis. Dx. $=$ Age Disability Diagnosed $; \mathrm{CSI}=$ College Stress Inventory; SSA-R $=$ Social Support Appraisal-Revised scale; Inter. Term = Interaction Term; SSA-R X CSI = Social Support Appraisal-Revised scale multiplied by College Stress Inventory.

Hypothesis 7-Part C. Hypothesis seven, Part C stated: Perceived social support will moderate the impact of college stress (as measured by the CSI) in psychosocial-emotional adjustment to college (as measured by the SACQ) among first-year and second-year undergraduates with CID.

A hierarchical MRA was conducted to address hypothesis 7, Part C, using psychosocial-emotional adjustment to college (as measured by the SACQ-T) as the criterion. Socio-demographic control variables included gender, a biological status 
variable, and age disability diagnosed, a disability-related variable. In Step 1, the control variables of gender and age disability diagnosed were entered into the model. This set of socio-demographic variables combined, were statistically significant, $\mathrm{R}^{2}=.065, \Delta \mathrm{F}(2,100)=3.462, p=.035(p<.05)$. In Step 2, the predictor college stress (as measured by the CSI) was added to the model. This stress-related variable was statistically significant, $\Delta \mathrm{R}^{2}=.124, \Delta \mathrm{F}(1,99)=15.173(p<.001)$. Further, CSI contributed approximately $12 \%$ of the variance in SACQ scores. In Step 3, perceived social support (i.e., SSA-R) as a potential predictor was added to the model, and a significant main effect emerged, $\Delta R^{2}=.305, \Delta F(1,98)=58.992, \beta=.620(p<.001)$. Perceived social support was found to add significant predictive utility for psychosocial-emotional adjustment to college (as measured by the Student Adaptation to College Questionnaire [SACQ-T, Total score]). Moreover, this variable accounted for approximately $31 \%$ of the variance in psychosocial-emotional adjustment scores. In Step 4, the interaction term (i.e., SSA-R X CSI) was added to the model. Examination of the $F$ change statistic and weights in Table 15 indicate that the interaction of perceived social support with college stress was not a significant overall predictor of psychosocial-emotional adjustment to college (i.e., $\Delta R^{2}=.000, \Delta F[1,97]=.015$, $\beta=.010, p=.902[p>.05])$. Further, the interaction term failed to increase the amount of variance explained in college adjustment when compared to the variables entered in previous steps.

In summary, the results for hypothesis 7-C provide support for the direct (main) 
effect model of perceived social support in the relationship between college stress and psychosocial-emotional adjustment to college among first-year and second-year undergraduate students with CID's. Additionally, perceived social support was found to be the most important predictor in the regression model, and was associated with successful adjustment to college. However, there was no support for the moderator (interaction) model of perceived social support in the relationship between collegerelated stress and psychosocial-emotional adjustment to college. Therefore, hypothesis 7-C was not supported. 
Table 15

Hierarchical MRA for Moderating Effects of Perceived Social Support on the Predictors (College Stress; Perceived Social Support) as Measured by Psychosocial-Emotional Adjustment to College: An Interaction Model

\begin{tabular}{|c|c|c|c|c|c|c|}
\hline $\begin{array}{l}\text { Step \& Predictor } \\
\text { Variables }\end{array}$ & $\mathrm{R}^{2}$ & $\Delta \mathrm{R}^{2}$ & $B$ & $S E B$ & $\beta$ & $\Delta \mathrm{F}$ \\
\hline Step 1 & .065 & $.065^{*}$ & 47.652 & 2.738 & & $3.462 *$ \\
\hline $\begin{array}{l}\text { Gender, } \\
\text { Age Dis. Dx. }\end{array}$ & & & $\begin{array}{l}3.579 \\
-.280\end{array}$ & $\begin{array}{r}2.481 \\
.155\end{array}$ & $\begin{array}{r}.143 \\
-.180\end{array}$ & \\
\hline Step 2 & & & 45.198 & 2.638 & & \\
\hline $\begin{array}{l}\text { CSI } \\
15.173 * * *\end{array}$ & .189 & $.124 * * *$ & -.319 & .082 & $-.366 * * *$ & \\
\hline Step 3 & & & 43.387 & 2.108 & & \\
\hline $\begin{array}{l}\text { SSA-R } \\
58.992 * * *\end{array}$ & .494 & $.305^{* * *}$ & & $.079 * * *$ & $.601 * * *$ & \\
\hline Step 4 & & & 43.432 & 2.150 & & \\
\hline $\begin{array}{l}\text { Int. Term: } \\
\text { SSA-R X CSI }\end{array}$ & .494 & .000 & .001 & .006 & .010 & .015 \\
\hline
\end{tabular}

$* p<.05 ; * * * p<.001$

Note. Age Dis. Dx. = Age Disability Diagnosed CSI $=$ College Stress Inventory; SSA $-\mathrm{R}=$ Social Support Appraisal-Revised scale; Int. Term: = Interaction Term: SSA-R x CSI = Social Support Appraisal-Revised scale multiplied by College Stress Inventory.

Hypothesis 8, Part A. Hypothesis eight, Part A stated: Perceived social support will moderate the impact of disability-related functional limitations(i.e., increased functional limitations, as measured by the DFLS) in adjustment to college (as measured by life satisfaction) among first-year and second-year undergraduates with CID.

A hierarchical MRA was conducted to address hypothesis 8, Part A, using life satisfaction as the criterion. Socio-demographic control variables included gender, a 
biological status variable, age at disability diagnosis, a disability-related variable, and hours employed, a social-related variable. In Step 1, the control variables (i.e., gender, age disability diagnosed, and hours employed) were first entered. This model was not statistically significant $\left(R^{2}=.068, p=.071(p>.05)\right.$. In Step 2 , the disability-related predictor variable, functional limitations (DFLS), was added to the model, but was not statistically significant $\left(\Delta R^{2}=.008, \Delta \mathrm{F}[1,98]=.822, p=.367[p>.05]\right)$. There was no support for functionality as a significant predictor of adjustment to college (as measured by life satisfaction) in the model. In Step 3, perceived social support as a potential predictor was added to the model. A significant main effect emerged, $\left.\Delta R^{2}=.401, \Delta F(1,97)=74.228, \beta=.677, p<.001\right)$. Further, perceived social support significantly increased the amount of variance explained in adjustment scores, accounting for approximately $40 \%$ of the variance in life satisfaction. Finally, in Step 4, the interaction term between perceived social support and disability-related functional limitations (SSA-R X DFLS) was added to the model. Examination of the $F$ change statistic and weights for the interaction analysis indicate that the interaction (SSA-R X DFLS) was not a significant overall predictor of college adjustment, $\Delta R^{2}=.013, \Delta F(1,96)=2.411, \beta=.121, p=.124(p>.05)$. Moreover, the interaction term was not found to significantly increase the amount of variance explained in college adjustment scores, when compared to that contributed by perceived social support entered in the previous step (i.e., Step 3). Findings (Table 16) provide support for the direct (main) effect model of perceived social support in the relationship between 
functional limitations and adjustment to college (as measured by life satisfaction) among first-year and second-year undergraduate students with CIDs. Moreover, perceived social support was the most important predictor in the regression model. Evidence that the relationship between perceived social support and functional limitations conforms to that predicted by a stress-interactive perspective was not statistically supported in the regression model. Therefore, Hypothesis 8, Part A, was not supported. 
Table 16

Hierarchical MRA for Moderating Effects of Perceived Social Support on the Predictors (Functional Limitations; Perceived Social Support) as Measured by Life Satisfaction: An Interaction Model.

\begin{tabular}{|c|c|c|c|c|c|c|}
\hline $\begin{array}{l}\text { Step and } \\
\text { Predictor Variable }\end{array}$ & $\mathrm{R}^{2}$ & $\Delta \mathrm{R}^{2}$ & $B$ & $S E B$ & $\beta$ & $\Delta \mathrm{F}$ \\
\hline Step 1 & & & 28.405 & 2.231 & & \\
\hline $\begin{array}{l}\text { Control Variables: } \\
\text { Gender } \\
\text { Age Dis. Dx. } \\
\text { Hours Employed }\end{array}$ & .068 & & & & & 2.419 \\
\hline Step 2 & & & 27.935 & 2.292 & & \\
\hline DFLS & .076 & .008 & -.170 & .187 & -.093 & .822 \\
\hline Step 3 & & & 25.817 & .466 & & \\
\hline SSA-R & $.477 * * *$ & $.401 * * *$ & $.466 * * *$ & .054 & $.677 * * *$ & $74.228 * * *$ \\
\hline Step 4 & & & 26.145 & 1.751 & & \\
\hline $\begin{array}{l}\text { Int. Term: } \\
\text { SSA-R X } \\
\text { DFLS }\end{array}$ & .489 & .013 & .016 & .010 & .121 & 2.411 \\
\hline
\end{tabular}

Note. Age Dis. Dx. = Age Disability Diagnosis; DFLS = Disability Functional Limitations Scale; SSA-R = Social Support Appraisal-Revised scale; Int. Term: = Interaction Term: SSA-R X DFLS = Perceived Social Support Appraisal-Revised scale multiplied by Disability Functional Limitations Scale.

Hypothesis 8, Part B. Hypothesis eight, Part B stated: Perceived social support will moderate the impact of functional limitations (as measured by the DFLS) in academic performance (as measured by GPA-S) in first-year and second-year undergraduate students with CID.

A hierarchical MRA was conducted to address the hypothesis using GPA-S as the criterion. Only one socio-demographic control variable was included in the model; age 
disability diagnosed, a disability-related variable. In Step 1, the control variable, age disability diagnosed was entered first. This variable contributed significantly to the prediction of GPA-S scores, $\mathrm{R}^{2}=.088, \mathrm{~F}(1,101)=9.750, p=.002(p<.01)$. In Step 2, the disability-related predictor, functionality, was added to the model. The contribution of functionality to the regression model however, was not statistically significant, $\Delta \mathrm{R}^{2}=.000, \Delta \mathrm{F}(1,100)=.002, p=.968(p>.05)$. In Step 3, the potential predictor variable, perceived social support, was added to the regression model. Findings show that perceived social support accounted for approximately $4 \%$ of the variance in academic performance scores. An examination of the $F$ change statistic and weights (Table 17) reveal the emergence of main effects. Perceived social support was a significant positive predictor of academic performance scores, with higher scores associated with increased academic success, $\Delta R^{2}=.044, \Delta F(1,99)=5.030, \beta=.224$, $p=.027(p<.05)$. Lastly, in Step 4, the interaction term between perceived social support and functionality (SSA-R X DFLS) was added to the model. Examination of the $F$ change statistic and final weights for the interaction analysis (Table 17) indicate that the interaction term was not a significant overall predictor of academic performance, $\Delta R^{2}=.012, \Delta F(1,98)=1.366, p=.245(p>.05)$. Further, the interaction was not found to significantly increase the amount of variance explained in academic performance, when compared to that contributed by perceived social support entered in the previous step (i.e., Step 3).

In summary, the results provide support for the direct (main) effect model of 
perceived social support in the relationship between functionality and academic adjustment to college. Additionally, perceived social support was found to be particularly associated with academic performance scores. However, there was no support for the moderator model of perceived social support in the relationship between functionality, a disability-related stressor, and academic performance, as measured by the GPA-S. Hypothesis 8-B, therefore, was not supported.

Table 17

Hierarchical MRA for Moderating Effects of Perceived Social Support on the Predictors (Functional Limitations; Perceived Social Support) as Measured by Academic Performance: An Interaction Model

\begin{tabular}{|c|c|c|c|c|c|c|}
\hline $\begin{array}{l}\text { Step and } \\
\text { Predictor Variables }\end{array}$ & $\mathrm{R}^{2}$ & $\Delta \mathrm{R}^{2}$ & B & SEB & $\beta$ & $\Delta \mathrm{F}$ \\
\hline Step 1 & & & 6.347 & .286 & & \\
\hline Age Dis. Dx. & $.088^{* *}$ & & -.060 & .019 & $-.297 * *$ & $9.750 * *$ \\
\hline Step 2 & & & 6.350 & .296 & & \\
\hline DFLS & .088 & .000 & .001 & .035 & .004 & .002 \\
\hline Step 3 & & & 6.240 & .294 & & \\
\hline SSA-R & $.132 *$ & $.044^{*}$ & .029 & .013 & $.224 *$ & $5.030^{*}$ \\
\hline Step 4 & & & 6.244 & .294 & & \\
\hline $\begin{array}{l}\text { Int. Term: } \\
\text { SSA-R x DFLS }\end{array}$ & .144 & .012 & -.003 & .002 & -.114 & 1.366 \\
\hline
\end{tabular}

$* p<.05 ; p * *<.01$

Note. Age Dis. Dx. = Age Disability Diagnosed DFLS $=$ Disability Functional Limitations Scale; SSA-R = Social Support Appraisal-Revised scale; Inter. Term = Interaction Term; Social Support Appraisal-Revised scale multiplied by Disability Functional Limitations Scale.

Hypothesis 8, Part-C. Hypothesis six, Part C stated: Perceived social support 
will moderate the impact of functional limitations (as measured by the DFLS) in psychosocial-emotional adjustment to college (as measured by the Student Adjustment to College Questionnaire [SACQ]) among first-year and second-year undergraduate students with CIDs.

A hierarchical MRA was conducted to address the hypothesis, using psychosocial adjustment to college (as measured by the Student Adaptation to College Questionnaire [SACQ] as the criterion. Socio-demographic control variables included gender, a person-related biological status variable, and age disability diagnosed, a disabilityrelated variable. In Step 1, the control variables (i.e., gender, age disability diagnosed) were entered. These variables combined, were statistically significant, $R^{2}=.065$, $\Delta F(2,100)=3.462, p=.035(p<.05)$. In Step 2 , the predictor variable, functionality (higher scores indicate a higher level of functional limitations), was added to the model. The $\Delta \mathrm{R}^{2}$ was not statistically significant (i.e., $\Delta R^{2}=.021, \Delta F[1,99]=2.287$, $p=.134[p>.05])$. Consequently, there was no support for functional limitation as a significant predictor of psychosocial-emotional adjustment to college in this model. In Step 3, perceived social support as a potential predictor was added to the model. A significant main effect emerged, $\left.\Delta R^{2}=.381, \Delta F(1,98)=70.030, p<.001\right)$, indicating perceived social support as a positive predictor of college adjustment scores, with higher scores associated with increased psychosocial-emotional adjustment. Moreover, perceived social support contributed approximately $38 \%$ of the variance in college adjustment scores. Examination of the $F$ change statistic and weights in Table 18 reveal 
that perceived social support was the most important predictor in the regression model, adding significant predictive utility for college adjustment, as measured by the SACQ. Finally, in Step 4, the interaction term between perceived social support and functional limitations (SSA-R X DFLS) was added to the regression model. Results of the interaction analysis are presented in Table 18. Examination of the $F$ change statistic and weights indicate that the interaction of perceived social support with functional limitations, was not a significant overall predictor of college adjustment $\left(\Delta R^{2}=.001\right.$, $\Delta F[1,97]=.041, \beta=-.016, p=.840[p>.05])$. Further, the interaction term did not significantly increase the amount of variance explained in psychosocial-emotional adjustment to college scores, when compared to that contributed by perceived social support, entered in the previous step (i.e., Step 3).

In summary, the results provide support for the direct (main) effect model of perceived social support in the relationship between functional limitations and psychosocial adjustment to college. Moreover, perceived social support was particularly associated with college adjustment, as measured by the SACQ-T (Total scale scores). However, there was no support for the buffer (interaction) model of perceived social support in the relationship between functional limitations, a disability-related stressor, and psychosocial-emotional adjustment to college among first-year and second-year undergraduates with CID. Hypothesis 8-C, therefore, was not supported. 
Table 18

Hierarchical MRA for Moderating Effects of Perceived Social Support on the Predictors (Functional Limitations; Perceived Social Support) as Measured by Psychosocial-Emotional Adjustment to College: An Interaction Model

\begin{tabular}{|c|c|c|c|c|c|c|}
\hline $\begin{array}{l}\text { Step and } \\
\text { Predictor Variable }\end{array}$ & $\mathrm{R}^{2}$ & $\Delta \mathrm{R}^{2}$ & $B$ & $S E B$ & $\beta$ & $\Delta \mathrm{F}$ \\
\hline Step 1 & & & 47.652 & 2.738 & & \\
\hline $\begin{array}{l}\text { Demographic } \\
\text { Control Variables: }\end{array}$ & $.065^{*}$ & & & & & $3.462 *$ \\
\hline Gender & & & 3.579 & 2.481 & .143 & \\
\hline Age Dis. Dx. & & & -.280 & .155 & -.180 & \\
\hline Step 2 & & & 46.431 & 2.838 & & \\
\hline DFLS & .086 & .021 & -.412 & .272 & -.153 & 2.287 \\
\hline Step 3 & & & 44.346 & 2.192 & & \\
\hline SSA-R & .467 & $.381 * * *$ & .666 & .080 & $.658 * * *$ & 70.030 \\
\hline Step 4 & & & 44.304 & 2.213 & & \\
\hline $\begin{array}{l}\text { Int. Term: } \\
\text { SSA-R X DFLS }\end{array}$ & .467 & .000 & -.003 & .015 & -.016 & .041 \\
\hline
\end{tabular}

$* p<.05 ; * * * p<.001$

Note. Age Dis. Dx. = Age Disability Diagnosed; DFLS = Disability Functional Limitations Scale; SSA-R = Social Support Appraisal-Revised scale; Int. Term = Interaction Term; SSA-R X DFLS = Perceived Social Support-Revised scale multiplied by Disability Functional Limitations Scale.

\section{Exploratory Research Questions}

The outcomes of data analyses related to the five research questions are included in this section (section 4) of the study. Specifically, research questions 1 through 4 utilized bivariate correlation analyses to analyze possible relationships between variables. Three analyses were conducted for each research question; one for each 
criterion variable (i.e., Part A, life satisfaction; Part B, academic performance; Part C, psychosocial-emotional adjustment to college). Research question 5 utilized analysis of variance (ANOVA) regarding possible disability group differences (i.e., those students with physical disabilities, cognitive/neurocognitive disabilities, psychiatric disabilities) and the study's three criterion variables regarding adjustment to college.

Research Question 1, Part A. The first research question, Part A stated: To what extent is time since diagnosis of disability (i.e., duration of disability [DOD]) related to adjustment to college, as measured by life satisfaction, among first-year and secondyear undergraduate students with CID?

The Pearson $r$ correlation did not reveal a significant relationship between the Duration of Disability (DOD) variable and the life satisfaction (i.e., SWLS) criterion variable $(r=-.097)$. Therefore, research question 1, Part A, did not support a relationship between these variables. Table 19 suggests that duration of disability (i.e., DOD) was not significantly correlated with adjustment to college, as measured by life satisfaction (SWLS); one of three quality-of-life indicators used in the present study to measure adjustment to college.

Research Question 1, Part B: The first research question, Part B stated: To what extent is time since diagnosis of disability (i.e., duration of disability [DOD]) related to adjustment to college, as measured by academic adjustment [GPA-S], among first-year and second-year undergraduate students with CID?

The Pearson $r$ correlation did not reveal a significant relationship between DOD 
and the criterion variable measuring academic adjustment to college, GPA-S $(r=.157)$. Therefore, research question 1, Part B, did not support a relationship between these variables. Table 19 illustrates the relation between Duration of Disability and adjustment to college, as measured by academic adjustment (GPA-S); one of three quality-of-life indicators used in the current study to measure college adjustment. Research Question 1, Part C: The first research question, Part C stated: To what extent is time since diagnosis of disability (i.e., duration of disability [DOD]) related to adjustment to college, as measured by psychosocial-emotional adjustment to college (SACQ), among first-year and second-year undergraduate students with CID?

The Pearson $r$ correlation did not reveal a significant relationship between the DOD variable and the SACQ criterion variable $(r=.091)$. Therefore, research question 1, Part C, does not support a relationship between these variables. Table 19 illustrates the relation between duration of disability (i.e. DOD) and college adjustment, as measured by the psychosocial-emotional adjustment to college criterion variable (SACQ-T). 
Table 19

Correlations among Predictor (DOD) and Outcome Measures (SWLS, GPA-S, SACQ-T) in Sample $(N=103)$ of First-Year and Second-Year College Students with CID

DOD

SWLS

GPA-S

SACQ-T

.091

Note . DOD = Duration of Disability; SWLS = Satisfaction with Life Scale; GPA-S = Grade Point Average-Scale; SACQ-T = Student Adaptation to College Questionnaire-Total score.

Research Question 2, Part A. The second research question, Part A stated: To what extent is present chronological age related to college adjustment, as measured by Global Life Satisfaction (SWLS) among first-year and second-year undergraduate students with CID?

The Pearson $r$ correlation revealed a significant and negative relationship between the chronological age (age) variable and the life satisfaction (i.e., SWLS) criterion variable $(r=-.313, p<.01)$. Therefore, research question 2, Part A, found a bivariate relationship between age and life satisfaction. Table 20 illustrates the relation between chronological age and adjustment to college, as measured by life satisfaction (SWLS); one of three quality-of-life indicators used in the present study to measure college adjustment in first-year and second-year undergraduate students with CID.

Research Question 2, Part B. The second research question, Part B stated: To what extent is present chronological age related to academic performance, as measured 
by the GPA-S, among first-year and second-year undergraduate students with CIDs?

The Pearson $r$ correlation revealed a significant and negative relationship between the chronological age variable (age) and the GPA-S criterion variable $(r=-.214$, $p<.01$ ). Therefore, research question 2 , Part B, found a bivariate relationship. Table 20 illustrates the significant and negative relation between chronological age and academic performance, as measured by the grade point average-scale (GPA-S).

Research Question 2, Part C. The second research question, Part C stated: To what extent is present chronological age related to adjustment to college, as measured by psychosocial-emotional adjustment to college (SACQ), among first-year and secondyear undergraduate students with CID?

The Pearson $r$ correlation revealed a significant and negative relationship between the chronological age variable (age) and the SACQ-T college adjustment criterion variable $(r=-.173, p<.05)$. Therefore, research question 2 , Part $\mathrm{C}$, found a bivariate relationship. Table 20 illustrates the significant and negative relation between chronological age and psychosocial-emotional adjustment to college, as measured by the SACQ-T (Total scale score). 
Table 20

Correlations among Predictor (Chronological Age) and Outcome Measures (SWLS, GPA-S, \& SACQ-T) in Sample $(N=103)$ of First-Year and Second-Year College Students with CID

\begin{tabular}{ll}
\hline & Age \\
\hline SWLS & $-.313^{* *}$ \\
GPA-S & $-.214^{* *}$ \\
SACQ-T & $-.173^{*}$ \\
\hline
\end{tabular}

$* \mathrm{p}<.05 ; * * \mathrm{p}<.01$

Note. SWLS $=$ Satisfaction with Life Scale; GPA-S $=$ Grade Point Average-Scale; SACQ-T $=$ Student Adaptation to College Questionnaire-Total scale score.

Research Question 3, Part A. The third research question, Part A stated: To what extent is gender related to adjustment to college, as measured by life satisfaction (SWLS) among first-year and second-year undergraduate students with CID?

An independent sample t-test was conducted to determine if there were significant mean differences in adjustment, as measured by life satisfaction (SWLS), among male $(n=46)$ and female $(n=56)$ respondents. However, the independent sample t-test suggested no significant (i.e., mean score) differences in levels of adjustment for men and women, as measured by the SWLS criterion variable, $t(101)=.28, p=.77$ (two-tailed).

Research Question 3, Part B. The third research question, Part B stated: To what extent is gender related to adjustment to college, as measured by academic performance (GPA-S), among first-year and second-year undergraduate students with CID? 
An independent sample t-test was conducted to determine the possibility of significant (i.e., mean score) differences in academic performance, among male $(n=46)$ and female $(n=56)$ respondents. Results of the independent sample t-test suggested no significant differences however, in academic performance among male and female respondents, $t(101)=.40, p=.69$ (two-tailed).

Research Question 3, Part C. The third research question, Part C stated: To what extent is gender related to adjustment to college, as measured by psychosocial-emotional adjustment to college (SACQ), among first-year and second-year undergraduate students with CID?

An independent sample t-test was performed to determine if there were significant mean differences between male $(n=46)$ and female $(n=56)$ respondents in college adjustment, as measured by psychosocial-emotional adaptation to college (SACQ-T). Results of the independent sample t-test suggested no significant differences in psychosocial-emotional adjustment to college, among male and female respondents, $t(101)=-1.89, p=.06$ (two-tailed).

Research Question 4, Part A. The fourth research question, Part A stated: To what extent is hours employed (per week) related to adjustment to college, as measured by life satisfaction (SWLS), among first-year and second-year undergraduate students with CID?

The Pearson $r$ correlation revealed a negative and significant bivariate correlation between the hours employed (per week) variable and the life satisfaction (i.e., SWLS) 
criterion variable $(r=-.172, p<.05)$. Table 21 suggests that hours employed was significantly and inversely associated with adjustment to college, as measured by life satisfaction (i.e., SWLS); one of three QoL indicators used in the current study to measure college adjustment.

Research Question 4, Part B. The fourth research question, Part B stated: To what extent is hours employed (per week) related to academic performance, as measured by GPA-S, among first-year and second-year undergraduate students with CID?

The Pearson $r$ correlation suggested no significant association between the hours employed (per week) variable and academic performance $(r=-.062, p>.05)$, as measured by GPA-S, among first-year and second-year undergraduate students with CID. Therefore, research question 4, Part B, found no bivariate relationship between hours employed and academic performance (i.e., GPA-S). Table 21 illustrates that hours employed was not significantly correlated with academic performance, as measured by the GPA-S.

Research Question 4, Part C. The fourth research question, Part C stated: To what extent is hours employed (per week) related to psychosocial-emotional adjustment to college, as measured by SACQ-T, among first-year and second-year undergraduate students with CID?

The Pearson $r$ correlation did not reveal a significant relationship between hours employed and the psychosocial-emotional adjustment to college (i.e., SACQ-T) criterion variable ( $r=-.092, p>.05)$. Therefore, research question 4, Part C, suggests 
no bivariate relationship. Table 21 illustrates that hours employed was not significantly correlated with psychosocial-emotional adjustment to college.

Table 21

Correlations among Predictor (Hours Employed) and SWLS, GPA-S, \& SACQ-T in Sample $(N=102)$ of First-Year and Second-Year College Students with CID

Hours Employed (per week)

\begin{tabular}{lc}
\hline SWLS & $-.172^{*}$ \\
GPA-S & -.062 \\
SACQ-T & -.092 \\
\hline
\end{tabular}

$* \mathrm{p}<.05$

Note. SWLS $=$ Satisfaction with Life Scale; GPA-S = Grade Point Average-Scale; SACQ-T = Student Adaptation to College Questionnaire-Total score.

Research Question 5. The fifth research question stated: Are there significant disability group membership differences (i.e., physical disability group, cognitive/neurocognitive disability group, psychiatric disability group) as related to the study's three criterion measures of college adaptation (i.e., life satisfaction [Satisfaction with Life Scale, SWLS]; academic performance [Grade Point Average-Scale, GPA-S]; psychosocial-emotional adjustment to college [Student Adaptation to College Questionnaire, SACQ])?

To address research question 5, one way analysis of variance (i.e., ANOVA) was conducted with follow-up post-hoc Turkey's test to compare multiple group means. Results of the ANOVA with post-hoc Turkey HSD revealed no significant group differences between any of the three pairs of means for each of the study's 
adaptation-associated criterion variables (i.e., life satisfaction [SWLS], academic adjustment [GPA-S], psychosocial adjustment to college [SACQ-T]). Specific results include: $\mathrm{SWLS}=F(2,100)=2.505, p>.05 ; \mathrm{GPA}-\mathrm{S}=F(2,100)=2.085, p>.05$; and, SACQ-T $=F(2,100)=2.097, p>.05$. The post-hoc multiple comparisons revealed no significant differences between any of the three pairs of means for each dependent (i.e., outcome) variable (i.e., life satisfaction [SWLS], academic adjustment [GPA-S], and psychosocial-emotional adjustment to college [SACQ-T].

\section{Sub-Aim of Study}

Lastly, section 5 of the chapter pertains to the current study's one sub-aim. The sub-aim and final research question uses hierarchical MRA to assess the possible contribution to the variance by the disengagement coping variable (i.e., D-COPE) and the study's three criterion variables. Three MRA's were conducted; one for each criterion variable (Part A, life satisfaction [SWLS]; Part B, academic performance [GPA-S]; and Part C, psychosocial-environmental adjustment to college [SACQ-T].

Research Question 5, Part A. The fifth research question, Part A stated: To what extent does disengagement coping contribute to the variance in adjustment to college, as measured by life satisfaction (SWLS), among first-year and second-year undergraduate students with CID, after controlling for relevant demographic factors?

A hierarchical MRA was conducted to address research question 5, Part A, using life satisfaction (SWLS) as the criterion variable. Socio-demographic control variables included age disability diagnosed, a disability-related variable, and hours employed, a 
vocation-related variable. In Step 1, the control variables (i.e., age disability diagnosed; hours employed) were entered first. These variables combined, were statistically significant $\left(R^{2}=.063, \Delta F[2,100]=3.390, p=.038[p<.05]\right)$. In Step 2 , the predictor variable, disengagement coping (i.e., D-COPE), was added to the regression model. The $\Delta R^{2}$ was statistically significant $\left(\Delta R^{2}=.526, \Delta F[1,99]=126.807, p=.001[p \leq .001]\right)$. Furthermore, examination of Table 22 reveals that D-COPE accounted for approximately $53 \%$ of the variance in adjustment to college scores, as measured by the SWLS. This finding suggests that disengagement coping added significant predictive utility for college adjustment, as measured by life satisfaction, a quality-of-life indicator used in the present study.

Table 22

Hierarchical Multiple Regression of Disengagement Coping (D-COPE) on Life Satisfaction (SWLS) in the Sample $(N=103)$ of First-Year and Second-Year College Students with CID

\begin{tabular}{|c|c|c|c|c|c|c|}
\hline & $\begin{array}{l}\text { Criterion } \\
\text { Variable }\end{array}$ & $\begin{array}{l}\text { Predictor } \\
\text { Variable }\end{array}$ & $R^{2}$ & $\Delta R^{2}$ & $\beta$ & $t$ \\
\hline Step 1 & SWLS & Age Dis. Dx. & .063 & .063 & & $13.999 *$ \\
\hline Step 2 & SWLS & D-COPE & .589 & .526 & -.748 & $-11.261 * * *$ \\
\hline \multicolumn{7}{|c|}{$* p<.05 ; * * * p \leq .001$} \\
\hline \multicolumn{7}{|c|}{$\begin{array}{l}\text { Note. } \text { Age Dis. Dx. = Age at Disability Diagnosis; Hrs. Emp. = Hours Employed (per week); } \\
\text { D-COPE = Disengagement Coping; SWLS = Satisfaction with Life Scale. }\end{array}$} \\
\hline
\end{tabular}


as measured by academic performance (GPA-S), in first-year and second-year college students with CID?

A hierarchical MRA was conducted to address research question 5, Part B. The GPA-S (Total score scale) was used for measuring academic adjustment, an adaptation-associated criterion variable. One relevant socio-demographic variable was included in the analyses, age disability diagnosed, a disability-related variable. In Step 1, the disability-related control variable, age disability diagnosed, was entered into the model first. This variable was statistically significant, $R^{2}=.088, \Delta F(1,101)=9.750$, $p=.002(p<.05)$. In Step 2, the predictor variable, disengagement coping (i.e., D-COPE), was added to the regression model. The $\Delta R^{2}$ was statistically significant $\left(\Delta R^{2}=.223, \Delta F[1,100]=32.275,[p<.001]\right)$. As reported in Table 23, the D-COPE variable accounted for approximately $22 \%$ of the variance in academic performance scores (i.e., GPA-S). Results suggest that the predictor variable, disengagement coping, added significant predictive utility for academic performance, as measured by GPA-S. 
Table 23

Hierarchical Multiple Regression of Disengagement Coping (D-COPE) on Academic Performance $(G P A-S)$ in the Sample $(N=103)$ of First-Year and Second-Year College Students with CID

\begin{tabular}{|c|c|c|c|c|c|c|}
\hline & $\begin{array}{l}\text { Criterion } \\
\text { Variable }\end{array}$ & $\begin{array}{l}\text { Predictor } \\
\text { Variable }\end{array}$ & $R^{2}$ & $\Delta R^{2}$ & $\beta$ & $t$ \\
\hline Step 1 & GPA-S & Age Dis. Dx. & .088 & .088 & & $-3.123 * *$ \\
\hline Step 2 & GPA-S & D-COPE & .311 & $.223 * * *$ & -.473 & $-5.681 * * *$ \\
\hline
\end{tabular}

$* * p<.05 ; * * * p<.001$

Note. Age Dis. Dx . = Age Disability Diagnosed; D-COPE = Disengagement Coping; GPA-S = Grade Point Average-Scale.

Research Question 5, Part C. The fifth research question, Part C stated: To what extent does disengagement coping contribute to the variance in college adjustment, as measured by psychosocial-emotional adjustment to college (i.e., Student Adjustment to College Questionnaire [SACQ]), among first-year and second-year undergraduate students with CID?

A hierarchical MRA was conducted to address research question 5, Part C, using psychosocial-emotional adjustment to college (as measured by the SACQ-T), as the criterion. Socio-demographic control variables included gender, a person-related biological status variable, and age disability diagnosed, a disability-related variable. In Step 1, the control variables (i.e., gender, age disability diagnosed) were entered into the model. These variables combined, were statistically significant, $R^{2}=.065$, $\Delta F(2,100)=3.462, p=.035(p<.05)$. In Step 2 , the predictor variable, disengagement coping (i.e., D-COPE), was added to the regression model. The $\Delta R^{2}$ was statistically 
significant (i.e., $\left.\Delta R^{2}=.357, \Delta F[1,99]=61.193, \beta=.143, p=.001[p \leq .001]\right)$.

Findings reported in Table 24 reveal that D-COPE accounted for approximately $36 \%$ of the variance in SACQ-T scores. This result suggests that disengagement coping added significant predictive utility for college adjustment, as measured by the SACQ-T.

Table 24

Hierarchical Multiple Regression of Disengagement Coping (D-COPE) on Psychosocial-Emotional Adjustment to College $(S A C Q-T)$ in the Sample $(N=103)$ of First-Year and Second-Year College Students with CID

\begin{tabular}{|c|c|c|c|c|c|c|}
\hline & $\begin{array}{l}\text { Criterion } \\
\text { Variable }\end{array}$ & $\begin{array}{l}\text { Predictor } \\
\text { Variable }\end{array}$ & $R^{2}$ & $\Delta R^{2}$ & $\beta$ & $t$ \\
\hline Step 1 & SACQ-T & $\begin{array}{l}\text { Age Dis. Dx. } \\
\& \text { Gender }\end{array}$ & .065 & .065 & & $17.406 * *$ \\
\hline Step 2 & SACQ-T & D-COPE & .422 & .357 & -.600 & $-7.823 * * *$ \\
\hline
\end{tabular}

$* * p<.05 ; * * * p \leq .001$

Note. Age Dis. Dx. = Age Disability Diagnosed; D-COPE = Disengagement Coping; GPA-S = Grade Point Average-Scale; SACQ-T = Student Adaptation to College Questionnaire (Total score).

Sub-Aim: Summary. In answering the one sub-aim research question (Part A, Part B, Part C), disengagement coping contributed to the variance in all three criterion measures of college adjustment: life satisfaction (53\%), academic performance (22\%), and psychosocial-emotional adjustment to college (36\%). Results suggested disengagement coping significantly added to the prediction of college adjustment, after controlling for relevant socio-demographic factors (i.e., gender; age disability diagnosed), in first-year and second-year undergraduate students with varying chronic 
illness and disability conditions. Therefore, in the current study, it was found that disengagement coping (i.e., D-COPE) predicted poorer adjustment to college outcomes, as measured by life satisfaction (i.e., SWLS), academic performance (i.e., GPA-S), and psychosocial-emotional adjustment to college (i.e., SACQ-T). 


\section{Chapter V}

\section{Discussion}

Many variables can impact students in their transition and adjustment to the college environment. This study explored relationships among college stress, functional limitations, coping strategies, and perceived social support in adjustment to college among 103 first-year and second-year undergraduate students with disabilities, guided by a quality-of-life framework of adaptation. Of particular interest were the potential stress-interacting effects of engagement coping and perceived social support (each) upon adjustment outcomes. An exploration of these coping strategies may be helpful in guiding rehabilitation and education professionals to the selection of therapeutic interventions that will most optimally promote successful college adjustment for students with disabilities.

Three specific research aims, along with one sub-aim, were included in the study. The first aim of this investigation highlighted the use of bivariate correlational analysis to examine the proposed link between each of the study's four predictor variables (college stress; functionality [i.e., increased level of functional limitations]; engagement coping strategies; perceived social support) and three adaptation-associated criterion measures of college adjustment (i.e., life satisfaction, academic performance, psychosocial-emotional adjustment to college). The second aim of the study, and also the primary aim, was to examine possible interactive effects of engagement coping strategies and perceived social support (each) in adaptation to college, using 
hierarchical multiple regression analysis. The third aim of the study was to explore several socio-demographic variables (personal-related [i.e., gender, respondents' present age], vocational-related [i.e., hours employed, per week], and disability-related [i.e., duration of disability]) and their possible relationship to the study's three adaptation-associated criterion variables in adjustment to college. Also included in the third aim was the exploration of possible disability group differences (i.e., physical disability group membership; cognitive/neurocognitive disability group membership; and, psychiatric disability group membership) as related to the study's three criterion variables. The last aim, also representing this exploratory study's sub-aim, was the investigation of disengagement coping and its possible contribution to the variance, in the study's adaptation-associated college outcomes.

This study's final chapter, Chapter V, contains five sections. The first section provides a discussion of the research findings, and is subdivided accordingly by each aim of the study. The second section provides a discussion of study highlights. The third section reviews limitations of the study. Recommendations suggested for future research are then highlighted. Lastly, section five provides a discussion of relevant counseling interventions based on findings of the study.

\section{Findings of the Study}

\section{First Study Aim}

The first aim of the study was to examine relationships among the study's four predictor variables: (a) college stress, (b) functional limitations, (c) engagement coping 
strategies, and (d) perceived social support, and three QoL outcome indices of adaptation to college (i.e., life satisfaction, academic performance, psychosocial-emotional adjustment to college).

College stress and adaptation. As predicted, higher levels of reported college stress were significantly related to lower levels of life satisfaction, academic performance, and psychosocial-emotional adjustment to college. This finding was overwhelmingly consistent with previous research of the general postsecondary student population, directly linking college stress to theoretically relevant outcome criteria, such as life satisfaction (e.g., Coffman \& Gilligan, 2002; DeMakis \& McAdams, 1994; Saber et al., 2012; Solberg et al.,1994; Yalcin, 2011), academic performance (e.g., Baker, 2003; Dziegielewski et al., 2004; Edwards \& Trimble, 1992; Felsten \& Wilcox, 1992; Gall et al., 2000; Macan et al., 1990; Misra \& McKean, 2000; Morosanu et al., 2010; Pritchard \& Wilson, 2003; Solberg et al., 1994; Struthers et al., 2000) and psychosocial-emotional college adjustment (e.g., Baker \& Siryk, 1984; Brooks \& DuBois, 1995; Compas et al.,1986; Felsten \& Wilcox, 1992; Gerdes \& Mallinckrodt, 1994; Herrington et al., 2005). The findings were also consistent with the few studies examining the relation between college stress and measures of college adaptation in disabled student postsecondary populations (e.g., Kerr et al., 2004; Sanders \& DuBois, 1996).

Functionality and adaptation. In this exploratory study, functionality (i.e., more pronounced functional limitations), a disability-related variable, was found to be 
significantly and negatively related to college adjustment, as measured by psychosocial-emotional adjustment to college (SACQ). No association was found however, between level of functionality and the study's two other outcome indicators of college adjustment: life satisfaction (as measured by the SWLS) and academic performance (as measured by the GPA-S).

The stress-moderating hypothesis based on the broader stress and coping literature (e.g., Aspinwall \& Taylor, 1992; Compas et al., 2001; Lazarus \& Folkman, 1984;

Cohen \& Wills, 1985; Dunkel-Schetter, \& Wortman, 1981; Lewis \& Frydenberg, 2004; Terry, 1989) may help to explain the lack of significant correlation between functionality (i.e., more pronounced functional limitations) and adjustment to college, as measured by life satisfaction. That is, the availability of engagement coping strategies may have served to reduce the effects of heightened levels of disability-related functional limitations on adjustment, as measured by life satisfaction (see statistical analysis [Chapter IV] and discussion [Chapter V], hypothesis 6-A). Nonetheless, the discovery of a negative association between functionality (i.e., more pronounced functional limitations) and psychosocial-emotional college adjustment was an indication that adaptation to college among first-year and second-year undergraduate students with disabilities involved a disability-related characteristic that may negatively influence the pursuit of educational goals. It remains extremely important, therefore, for researchers to continue to explore the role of disability-related variables (such as stigma, chronic pain, chronic fatigue, endurance) in future studies investigating college 
adjustment among students with disabilities.

Engagement coping and adaptation. As in prior research with nondisabled postsecondary students (e.g., Appelhans \& Schmeck, 2002; Aspinwall \& Taylor, 1992; Baker, 2003; Calvete \& Connor-Smith, 2006; Dyson \& Renk, 2006; Kariv \& Heiman, 2005; Julal, 2012; Lent et al., 2002; Misra \& McKean, 2000; Struthers et al., 2000), engagement coping was found in this sample of first-year and second-year undergraduate students with disabilities, to have a significant association with ratings reflecting successful adjustment to college (i.e., as measured by life satisfaction, academic performance, psychosocial-emotional adjustment). Engagement-type coping efforts involve behavioral, affective, and cognitive aspects of coping, such as active planning, seeking instrumental support for problem solving, and positive reframing (Tobin et al., 1989). Although these findings replicate previous empirical work in coping and adjustment, they are nonetheless uniquely meaningful, because they add to the paucity of literature examining coping strategies in a college population of students with disabilities.

Perceived social support and adaptation. As hypothesized, perceived social support was significantly and positively related to college adaptation, as measured by life satisfaction, academic performance, and psychosocial-emotional adjustment to college. This finding was consistent with previous research in samples of the general undergraduate student population, in which first-year and second-year postsecondary students reporting higher levels of perceived social support also reported higher levels 
of life satisfaction (e.g., Coffman \& Gilligan, 2002; Demakis \& McAdams, 1994;

Diener \& Fujita, 1995; Yalcin, 2011), academic performance (e.g., Solberg et al., 1994; Tinto, 1993; Wilkes \& Spivey, 2010) and psychosocial-emotional adjustment to college (e.g., Tao et al., 2000). Perceived social support obtained from family, peers, and other significant individuals, therefore, played a significant and beneficial role in the lives of this sample of college students with disabilities. Findings were also consistent with the paucity of previous studies suggesting a significant and positive relationship between perceived social support and college adjustment outcomes in postsecondary students with disabilities (Murray et al., 2012; Sanders \& DuBois, 1996; Winterowd, Street, \& Boswell, 1998).

\section{Second Study Aim}

The second aim of this study was to examine predicted moderating relationships involving one college-related predictor (i.e., college stress), one disability-related predictor (i.e., functionality, as measured by more pronounced functional limitations), and two moderators, including engagement coping and perceived social support (each) in adaptation to college. The QoL model of Psychosocial Adaptation to Chronic Illness and Disability (i.e., QoL-PACID) considers engagement-type strategies and perceived social support as internally anchored coping efforts that can influence psychosocial adaptive outcomes (Livneh \& Martz, 2012). Such outcomes were measured in the current study by three QoL indices of college adjustment: Namely, life satisfaction (SWLS; Diener et al., 1985), academic performance (GPA-S), and 
psychosocial-emotional adjustment to college (SACQ; Baker \& Siryk, 1999). A total of twelve predictions (hypotheses) were made. The discussion of these findings is subdivided into two parts; those hypotheses predicting: (a) engagement coping as a moderator (hypotheses 5 through 6); and, those hypotheses predicting (b) perceived social support as a moderator (hypotheses 7 through 8).

Engagement coping as a moderator. By and large, engagement coping (i.e., E-Cope) was not found to moderate the predicted stress-adjustment relations in this sample. The exception to this conclusion was the role engagement coping strategies played in moderating the relationship between disability-related functionality (i.e., as measured by the DFLS) and college adjustment, as measured by life satisfaction (i.e., SWLS). Findings revealed that increased levels of engagement coping (i.e., high E-COPE) were associated with significantly better adjustment to college (i.e., higher SWLS scores) than lower levels of engagement coping (i.e., low E-COPE), but only under the condition of more severe functionality level (i.e., more pronounced functional limitations; high DFLS scores). Disability-related functionality (i.e., DFLS) as a stand-alone variable, did not add to the variance in life satisfaction scores, after controlling for relevant socio-demographic variables. However, when viewed within the context of engagement coping (i.e., in the interaction), functionality (more pronounced functional limitations) contributed to the prediction of better adaptation to college (i.e., higher life satisfaction scores).

The nature of this significant interaction suggested that higher levels of 
coping (i.e., high E-COPE scores) played a significant role in the stress-adjustment relation, but only under the condition of high stress (i.e., high DFLS scores). Such strategies reflected an active, goal-oriented, and reality-based approach to coping, in which individuals acknowledged their problems and found alternative, successful ways of resolving them. In this exploratory study, engagement coping was empirically recognized as an effective intrapersonal variable that moderated disability-related functional limitations, thus maximizing successful adaptation (as measured by life satisfaction) to college among first-year and second-year undergraduate students with a wide variety of disabilities. It can also be one of the most efficient interventions that rehabilitation professionals can provide to clients choosing a rehabilitation plan that requires postsecondary education to achieve vocational goals.

Although there was limited support for the interacting role of engagement coping, results provided overwhelming support for the main effects model in the relationship between engagement coping strategies and the study's three QoL criterion variables measuring adaptation to college (i.e., life satisfaction [SWLS], academic performance [GPA-S], and psychosocial-emotional adjustment to college [SACQ]) in first-year and second-year undergraduate students with a wide variety of physical, cognitive/neurocognitive, and psychiatric disabilities. That is, significant main effects were observed in all 6 of the 6 regression analyses, and significant interaction effects were observed in 1 of the 6 analyses. Thus, in general, although these findings supported both a main and moderating effect, they were more heavily weighted towards 
main effects. The main effects model proposes that coping has uniform, beneficial effects on adaptive outcomes, regardless of the stressfulness or nature of the problem(s) being faced (Terry, 1989). Despite the inconclusive findings of the current study, many prominent researchers continue to endorse the role of coping (i.e., those strategies reflecting an engagement-type/problem-solving perspective) as an effective moderator against stressful life events (e.g., Compas et al., 2001; Connor-Smith \& Compas, 2004; Folkman, Lazarus, Dunkel-Schetter, DeLongis, \& Gruen, 1986; Lazarus, 1999; Lazarus \& Launier, 1978; Moos \& Holahan, 2003; Ptacek \& Pierce, 2003; Terry, 1989).

The lack of consistent support for engagement coping as a moderating variable in the current study may be attributable to several factors. First, it may be the case that other unexplored and unknown moderating variables were responsible for the outcomes in this study, such as personality characteristics (Carver et al., 1989). Personality researchers have found that a number of stable individual differences predispose individuals to use certain coping strategies (Carver et al., 1989; Conner-Smith \& Flachsbart, 2007; DeNeve, 1999; Ferguson, 2001; McFatter, 1994; Scheier, Weintraub, \& Carver, 1986). For example, Ferguson (2001) found that neuroticism and introversion were associated with ineffective coping behaviors, such as denial. Optimistic individuals, on the other hand, were found to engage in active, problem-focused coping or strategies that could alter the problematic situation, leading to more effective resolution of the stressful situation. Optimistic individuals also tend to seek out social support, engage in positive reappraisal of adverse events, and feel as if they have the 
resources to overcome stressful situations. These are all factors that can help buffer against the effects of negative life events, thereby influencing well-being and other adaptive outcomes (Carver et al., 1989; Connor-Smith \& Flachsbart, 2007;

Scheier et al., 1986). Further empirical research would profit by examining the possible interaction effects of stable, personality traits (e.g., optimism, neuroticism, hope, introversion) with life stress and psychosocial outcomes in adjustment to college among undergraduate students with disabilities.

Second, consideration must be given to the possibility of coping as a mediating variable. The mediator hypothesis assumes that the manner by which an individual understands or reacts to stress determines the impact of the stressor on adaptive outcomes (Lazarus \& Folkman, 1984). The stressful event, therefore, determines the response, which in turn contributes to the relationship between stressor and psychosocial outcome. If supported empirically, effective engagement coping efforts may indirectly affect adaptive outcomes via a protective process of preventing or minimizing stressors through another variable, such as perceived social support. In fact, several studies found in the higher education literature suggest such a relationship (e.g., Brissette, Scheier, \& Carver, 2002; Calvete \& Connor-Smith, 2006; Chang \& Strunk, 1999; Tao et al., 2000).

For example, Calvete and Connor-Smith (2006) found that coping (engagement-type strategies) mediated relations between perceived social support and psychological symptoms in American $(N=349)$ and Spanish $(N=437)$ college 
students coping was measured by the 57-item Response to Stress Questionnaire (RSQ; Connor-Smith, Compas, Wadsworth, Thomsen, \& Saltzman, 2000). Three RSQ coping factors included Primary Control Coping, consisting of problem solving, emotional regulation, and emotional expression subscales; Secondary Control Coping, consisting of distraction, positive thinking, cognitive restructuring, and acceptance; and Disengagement Coping, consisting of avoidance, denial, and wishful thinking. Perceived social support was assessed by the Multidimensional Scale of Perceived Social Support (MSPSS; Zimet, Dahlem, Zimet, \& Farley, 1988). This scale consists of 12 items assessing support perceived to be available from family, friends, and significant others. The Social Stress Questionnaire (SSQ; Connor-Smith \& Compas, 2002), a brief measure assessing the number of negative interpersonal events experienced during the last six months, and the degree to which those events were perceived as stressful, was used to report social stress. Lastly, the Young Adult Self-Report (YASR; Achenbach, 1997), was used as an outcome indicator of psychosocial distress. YASR syndromes used in the study included the Anxious/Depressed syndrome (symptoms reflecting feeling sad, worthless, worried, and nervous); the Social Withdrawal syndrome (preferring to be alone, being secretive, and being socially isolated); and, the Aggressive Behavior syndrome (overtly aggressive behaviors, such as fighting, physical attacks, and verbal threats). The protective effect of perceived social support appeared to have decreased the use of harmful disengagement coping strategies (e.g., avoidance, withdrawal, denial) while increasing 
the use of beneficial engagement coping strategies. It was speculated by Calvete and Connor-Smith (2006) that students who felt emotionally supported from friends, family, and other important persons, were more likely to attempt to solve problems, actively express and regulate emotions, and find new ways to think about difficulties (i.e., engagement strategies), as well as to likely avoid or deny feelings or problems (i.e., disengagement strategies). Because the study was cross-sectional, however, it was not possible to demonstrate the direction of relations between perceived social support and coping.

Lastly, it is possible that the range of scores for engagement coping (i.e., E-COPE) was not wide enough to allow for an interactive factor to emerge. In the current study, the mean score of E-COPE was 27.84, and the standard deviation was 5.37 (i.e., $M=27.84 ; S D=5.37$ ). The range of scores for engagement coping (i.e., E-COPE) was 13 to 37 , minimum and maximum, respectively.

\section{Perceived Social Support}

The current study focused on developing insight regarding the moderating role of perceived social support in promoting adjustment to college in first-year and secondyear undergraduate college students. Gaining a better understanding of when and how perceived social support serves as a moderator is important for counseling professionals working with such students. Knowing that perceived social support can be particularly beneficial (given the potential college-and disability-related stressors a student with a disability may face) can help counseling professionals intervene more effectively by 
addressing the student's specific support needs. Working with the student to identify the helpful (or unhelpful) aspects of his or her support should be helpful in providing the student with the skills needed for effective coping. However, no evidence was found to support the stress-interacting hypothesis for perceived social support in the relation between selected predictor variables (i.e., college stress; functional limitations) and adaptation-associated indices of college adjustment (i.e., life satisfaction, academic performance, psychosocial-emotional adjustment to college) among first-year and second-year undergraduate students with disabilities.

Previous studies have suggested that perceived social support can ameliorate the potentially debilitating effects of stress, particularly when high levels of stress are faced. That is, the availability of supportive social relations, if needed, can help the individual better deal with stressful situations, resulting in greater psychosocial adjustment than individuals who perceive little or no available social support (e.g., Cairney, Boyle, Offord, \& Racine, 2003; Cohen \& Hoberman, 1983; Cropley \& Steptoe, 2005; Swift \& Wright, 2000).

While there was no evidence for the moderating model of perceived social support, results in the current study did weigh heavily towards supporting the main, direct effects model. Specifically, significant main effects were observed in 5 of the 6 regression analyses. Thus, the perceived availability of support appeared to mostly exert direct, beneficial effects in enhancing adaptation-associated college outcomes (i.e., life satisfaction, academic performance, psychosocial-emotional adjustment to college) 
among first-year and second-year undergraduate students with disabilities. Other studies also reveal support for the main effects model of perceived social support in college adjustment outcomes (e.g., Hinderlie \& Kenny, 2002; Murray et al., 2012;

Rodrequez et al., 2003; Solberg \& Villarreal, 1997; Winterowd, Street, \& Boswell, 1998). For example, Rodrequez et al. (2003) found no support for the moderating role of perceived support, but did find support for the main effects model in college adjustment among Latino students $(n=338)$.

One possible explanation for the lack of moderating effects in the current study may be related to the way in which perceived social support was conceptualized and measured. In the current study, perceived social support was conceptualized as a global, unitary entity, measured by one functional index of support: esteem support. In their review of the literature, Cohen and Wills (1985) suggest that social support functions should match the resources needed to cope with a specific type of stressor. These researchers imply that only specific (and appropriate) functional measures of support will show moderating/interacting effects. When faced with stressors that involve academic-related problems, for example, it may be that only the informational function of support (e.g., tutoring, academic advising, writing assistance) will serve to moderate its effects on college adjustment. Future research examining the potential interacting role of perceived support in the stress-adjustment relation should therefore include a measurement instrument that incorporates several different functional indexes of such support. For example, the Interpersonal Support Evaluation List (ISEL; 
Cohen \& Hoberman, 1983) examines four indexes of perceived support: esteem support (feeling valued, esteemed, and cared for), informational support (assistance in problem-solving), tangible support (the provision of financial aid, material resources, and/or in-kind assistance), and affiliation support (companionship). By assessing perceived support according to its specific functions, important differences in the adaptive nature of a variety of support functions may hopefully be captured. Indeed, other studies have supported the moderating role of perceived support, when specific functions of such support have been empirically investigated (e.g., Cohen \& Hoberman, 1983; Elliott et al., 1992; Murray et al., 2012; Swift \& Wright, 2000; Wilkes \& Spivey, 2010).

For example, a study by Swift and Wright (2000) hypothesized that specific functions of perceived support would moderate the effects of stressful life events on psychological distress among 60 college women who had experienced coercive sexual events and interpersonal stressors. Psychological distress, the criterion variable, was measured by the Symptoms Checklist-90-Revised Scale (SCL-90-R; Derogatis, 1977), a 90-item self-report inventory which assesses how distressed participants have been feeling during the past seven days. The composite score of the SCL-90-R was used. The ISEL (Cohen \& Hoberman, 1983) was used to measure perceived availability of social support. The study revealed either significant interactive effects, or a meaningful trend for interactive effects, in all functions of support measured by the ISEL.

Researchers (Cohen \& Wills, 1985; Licitra-Kleckler \& Waas, 1993) also suggest 
that in studies of moderating effects, the instrument used to measure social support may be too global, and therefore, not sensitive enough to detect higher-order interaction effects. Relevant to the current study, however, such research usually reveals main effects. A global (i.e., undifferentiated) measure of perceived social support, such as the Social Support Appraisals scale (SSA; Vaux et al., 1986) used in the current study, may have consequently been less successful in detecting interaction effects. In the Swift and Wright (2000) study, overall global perceived social support was also assessed for possible interactive effects. Global perceived social support did not serve as a moderator in any of the hypothesized relationships between stressful life events and psychological distress. However, main effects were detected. Findings by Swift and Wright (2000), therefore, support the contention by Licitra-Kleckler \& Waas (1993) that social support interactive effects cannot necessarily be established, when a global measure of support has been utilized by the researcher.

It is also possible that perceived social support was a proxy for some causal variable(s) with which support was highly correlated. Stable personality characteristics such as competence and sociability could have been plausible candidates. That is, it may have been that socially competent people were more capable of developing the perception of supportive relationships by effectively coping with stressful events or by performing effective coping behaviors. Hence, effects that might have been attributable to perceived support may have been partially or wholly attributable to personality traits such as competence and sociability that were highly correlated with social support 
(Ferguson, 2001). Studies using longitudinal prospective designs that include measures of variables such as social competence, sociability, extraversion, and neuroticism would be crucial in ruling out specific rival explanations for perceived social support effects.

\section{Third Study Aim}

The influence of socio-demographic variables was also investigated in the current study. These variables included: (a) duration of disability (i.e., disability-related), (b) chronological age of respondents (i.e., biologically-related), (c) gender (biologically-related), (d) hours employed (per week) (vocationally-related) and (e) possible difference in primary disability group membership (i.e., physical, cognitive/neurocognitive, psychiatric), as related to the study's three criterion measures of college adaptation (i.e., life satisfaction, academic performance, and psychosocial-emotional adjustment to college).

Duration of disability. Despite a growing body of research specifically addressing student adaptation to college for individuals with disabilities, relatively scant attention has been paid to the potential unique role of disability-related factors (Adams \& Proctor, 2010; Martz, 2004). Studies investigating (un)successful adaptation to college should therefore include a focus on variables related to the direct experience of chronic illness and disability. One such disability-related variable considered relevant in terms of its possible association with psychosocial adaptation outcomes, is the concept of duration of disability (DoD) (Livneh \& Martz, 2012). A large body of evidence accumulated on the role of $\mathrm{DoD}$ in relation to psychosocial adaptation to disability 
has yielded contradictory findings, however. While some studies in the rehabilitation psychology literature have suggested a trajectory of improved adaptation over time in individuals with certain medical conditions (i.e., spinal cord injury, cancer, multiple sclerosis) (Chase et al., 2000; Elfstrom, Kennedy, Lude, \& Taylor, 2007), others have revealed no such relation (e.g., Crisp, 1992; Hammell, 2004; Kennedy, Evans, \& Sandhu., 2009; Krause \& Crew, 1990; McNulty et al., 2004). The inconclusive picture of a relationship between DoD and adaptation-associated outcomes has led some researchers in the field of rehabilitation psychology to propose that relationships between the two may not necessarily be captured by a simple linear trend (Livneh \& Martz, 2012). In other words, there may be complex influences of mediating or moderating variables in the proposed relationships between $\mathrm{DoD}$ and adaptation-associated outcomes. According to Livneh and Martz (2012) such influences might include: the stability of a condition over time, pre-disability personality attributes, coping modes used, and outcome measures adopted by the researcher.

Findings of the current study revealed no significant relationships between DOD and adaptation to college (as measured by life satisfaction, academic adjustment, and psychosocial-emotional adjustment to college) in first-year and second-year undergraduate college students with disabilities. These findings may reflect the type of disabilities most commonly reported by respondents in the current study: conditions (i.e., cognitive/neurocognitive [45.2\%] and psychiatric [19.2\%]) that do not involve disability-related trauma to cope with. 
Chronological age. Descriptive analysis revealed that of the 103 respondents, the average age was 22 years-old, with an age range between 18 and 47 . While eighty-five percent of respondents were between ages 18 and 25, fifteen percent of respondents were between ages 26 and 47.

Bivariate analyses revealed significant and negative correlations between the chronological age variable (although its narrow range of values must be recognized) and the study's three adaptation-associated criterion measures (i.e., life satisfaction, academic adjustment, psychosocial-emotional adjustment to college). A study by Sanders and DuBois (1996) also reported a significant negative correlation between chronological age and ratings of total adjustment on the SACQ $(r=-.47 ; p<.05)$. As in the current study, college adaptation was investigated in an undergraduate population of students with disabilities. Findings from the Sanders and DuBois (1996) study must be interpreted cautiously however, due to its relatively small sample size $(n=32)$ limiting generalizability of the findings. Age as a socio-demographic characteristic should be explored in future studies examining college adjustment in lower division undergraduate students with disabilities, such as comparing first-year (i.e., freshman-level) and second-year (i.e., sophomore-level) students. However, it is recommended that a wider span of ages be achieved than that of the current study.

Gender. An independent samples t-test was performed to determine if there were statistically significant differences in terms of gender and college adjustment outcomes (i.e., life satisfaction, academic adjustment, psychosocial-emotional adjustment to 
college). Results suggested no significant differences in mean scores between male and female undergraduate students as measured by all three QoL indices of college adjustment among first-year and second-year undergraduate students with disabilities.

The independent samples t-test results are cautiously reported, as no other studies could be located in the literature to support the findings. Future studies should continue to explore the role of gender in adaptation to college among undergraduate college students with disabilities.

Hours employed (per week). Employment responsibilities (i.e., hours employed, per week) while attending college as a lower division undergraduate student has been found in past studies to adversely affect student retention (McKenzie \& Schweitzer, 2001; Russell \& Petrie, 1992). The influence of hours employed (per week), has often failed to be examined in studies of college student adjustment, however. In the current study, bivariate correlational analysis revealed a significant and inverse relationship between hours employed (per week) and adaptation to college, as measured by life satisfaction (i.e., SWLS). No other significant associations between hours employed and the study's other adaptation-associated criterion variables (i.e., academic adjustment [GPA-S]; psychosocial-emotional adjustment to college [SAQ-T]) were revealed. Findings suggested that employment responsibilities (while attending college) negatively influenced college adjustment, as measured by life satisfaction, in first-year and second-year undergraduate students with disabilities.

Although no previous studies could be located confirming a negative and 
significant correlation between hours employed and undergraduate student adjustment to college, as measured by life satisfaction (i.e., SWLS), a study by Hertel (2002) suggested a trend for such a relationship. In the Hertel (2002) study, the hours employed (per week) socio-demographic variable suggested a negative, but non-significant correlation to college adjustment (as measured by the Student Adaptation to College Questionnaire [i.e., SACQ; Baker \& Siryk, 1999]), in a random sample $(N=130)$ of first-generation and second-generation college students adjusting to college. No significant bivariate correlation between hours employed and the SACQ criterion variable, however, was found in the current study $(r=-.092, p>.05)$.

Disability-group differences. No studies could be located in the literature exploring possible disability-group differences in college adjustment (e.g., life satisfaction, academic performance, psychosocial-emotional adjustment) among students with CID. In an attempt to more fully understand if such group differences existed in the current study, an ANOVA with follow-up Turkey HSD test was conducted. For these analyses, students were grouped by three broad disability categories (Physical, Cognitive/Neurocognitive, Psychiatric). Group compositions included: physical disabilities (34.6\%), cognitive/neurocognitive disabilities (45.2\%) and psychiatric disabilities (19.2\%), respectively. No significant group differences between any of the three pairs of means for each of the study's adaptation-associated criterion variables (i.e., life satisfaction, academic adjustment, psychosocial-emotional adjustment to college) were suggested. These results should be considered tentative at 
best however, as more studies are needed to confirm these preliminary findings.

\section{Sub-Aim of Study}

Lastly, the one sub-aim of this exploratory study was to examine whether disengagement coping strategies contributed to the variance regarding all three criterion measures of college adaptation (i.e., life satisfaction [SWLS], academic adjustment [GPA-S], psychosocial adjustment to college [SACQ]), after controlling for relevant socio-demographic factors. Findings supported the direct, predictive role of disengagement coping in all three adaptation-associated outcome measures. Such coping efforts comprise an array of cognitive, affective, and/or behavioral strategies that are orientated away from the stressor or one's emotions or thoughts (e.g., denial, emotional venting, distraction, substance use, self-blame/criticism). Individuals who utilize these strategies seek to distance themselves from directly dealing with the stressful situation. The literature pertaining to the role of intrapersonal coping in student adaptation to college conclusively supports the current findings. Empirical investigations have consistently found disengagement-type coping strategies predictive of poorer adaptive outcomes among undergraduate student populations adjusting to college life (e.g., Aspinwall \& Taylor, 1992; Dyson \& Renk, 2006; Leong et al., 1997; Pierceall \& Keim, 2007; Saber et al., 2012; Struthers et al., 2000; Yum et al., 2005), including the paucity of studies focusing on students with disabilities (e.g., Heiman \& Kariv, 2004; Wodka \& Barakat, 2007). For example, Wodka \& Barakat (2007) found that disengagement-type coping predicted poorer college adjustment among 
first-year and second-year undergraduate students with physical chronic illness. Adjustment to college outcomes were measured using the total symptoms scores of the Beck Anxiety Inventory (BAI) (Beck \& Steer, 1993), and the Beck Depression Inventory-II (BDI-II) (Beck et al., 1996).

Although disengagement-type coping strategies are generally viewed as maladaptive, the literature suggests that some disengaged strategies may actually be helpful to individuals with disabilities in managing their day-to-day activities, if such strategies are employed soon after a crisis, and used for a limited amount of time (Holahan \& Moos, 1987). For example, the use of self-distraction (such as watching television instead of doing school work) for the acute management of a pain flair-up would be a beneficial coping response for students dealing with chronic pain. Reliance on such a coping strategy over time however, would likely be problematic, and lead to worse overall adaptive outcomes (e.g., low GPA, poor college adjustment).

\section{Discussion}

A college education has become a critically important component in both economic and overall quality-of-life for the American worker. In spite of the need for a college education, postsecondary attrition is high. Nearly one in four undergraduate students drop out of college within the first two years of matriculation. Most of these lower division students simply could not adjust to college life. For undergraduate students with disabilities, college adjustment is even more complicated due to the compounding presence of a disability. Although some students leave college for reasons 
beyond the control of the institution, most attrition is preventable (Tinto, 1993). As a result, factors that influence a student's ability to successfully adapt to college have received increased research attention in recent years.

The purpose of the current study was to explore relations among college stress, disability-related functional limitations, engagement coping, and perceived social support in adjustment to college among first-year and second-year undergraduate students with disabilities. Adaptation-associated outcomes were measured by life satisfaction, academic performance, and psychosocial-emotional adjustment to college. Bivariate analyses suggested that all four predictor variables were significantly associated with student adjustment, as measured by all three outcome measures. Hierarchical multiple regression supported mostly main effects for engagement coping and perceived social support while, mostly, failing to demonstrate moderator effects. The exception to this conclusion was the role engagement coping played in moderating the relationship between functional limitations and college adjustment, as measured by life satisfaction.

It must be noted that other variables not explored in this study could have had greater salience for understanding adaptation to college in students with disabilities. Academic motivation to learn, self-confidence, and a positive attitude toward the postsecondary institution, have all been found to be important psychosocial correlates of college adjustment in the general college population (Gerdes \& Mallinckrodt, 1994). Other prominent psychosocial variables found in the literature to predict college 
adjustment include locus of control, academic self-efficacy, and problem-solving skills. (Sanders \& DuBois, 1996). However, the extent to which these variables may apply to first-year and second-year undergraduate students with disabilities is largely unknown.

Research addressing socio-environmental factors may also offer key insight into understanding college adjustment among lower division undergraduate students with disabilities. In view of the additional resources and services that students with disabilities typically need to access, they may require larger formal social networks to adjust and be successful in college. Structural (i.e., quantitative) aspects of social support (e.g., network support), which were not considered in the current study, may, therefore, have significant relevance for this population. Research could also explore overall student satisfaction with disability resource support, as well as satisfaction with the various services provided to students with disabilities. Such studies would offer a unique contribution to the literature regarding the experiences and college adjustment of students with disabilities.

Campus climate factors may also offer valuable insights into understanding college adjustment among students with disabilities. Campus climate refers to a broad area that describes the overall social college environment, and rests on a continuum from unwelcoming to welcoming of students with disabilities. This environment is created by social and communal attitudes towards students with disabilities. The greatest impact of attitudes (positive or negative) toward students with disabilities tend to be those of postsecondary faculty and administrators (Vogel, Holt, Sligar, \& Leake, 
2008); the individuals who wield the greatest measure of power over students on college campuses. These attitudes can also come from fellow students without disabilities. Although highly influential, attitudes can be difficult to study from a quantitative perspective. Qualitative methodology stresses the process in which individuals create and give meanings to their social experience and lived realities (Heppner et al., 2008). Therefore, a qualitative research study on the topic of campus attitudes towards students with disabilities, would represent the best baseline starting point for exploring this phenomenon.

Of the three QoL outcome indicators used in the current study to assess student adjustment to college, the Student Adaptation to College Questionnaire (SACQ; Baker \& Siryk, 1999) represented the best measure to capture how well a student adapts to the demands of the college experience. There was no anticipated moderating effect however, between intrapersonal coping variables (i.e., engagement coping; perceived social support) and negative life events (i.e., college stress; functional limitations) in predicting psychosocial-emotional adjustment to college (as measured by the SACQ). This finding represented a disappointing aspect of the research findings. The authors of the SACQ (Baker \& Siryk, 1999) caution that the norms for the instrument are based on data from one postsecondary institution. Suitability of the normative data for other populations should therefore, not be taken for granted. This is particularly true for students whose cultural background differs significantly from that of the standardized sample, such as students with disabilities. Although the SACQ has been used quite 
successfully as an outcome indicator in hundreds of studies of college adjustment, the lack of normative data for students with disabilities is a specific limitation of the instrument that should be noted.

\section{Limitations}

The findings of this exploratory study must be interpreted with caution because of several important limitations. First, the sample of respondents were mostly single, and composed of first-year and second-year undergraduate college students with disabilities attending a public, four-year university. Respondents also represented a specific geographical area (i.e., Pacific Northwest) of the United States. In addition, the decidedly White sample rendered the results ethnic-specific. Respondents were also not randomly selected, possibly affecting representativeness of the population. The voluntary nature of participation, as well as relatively high adjustment scores may suggest that these respondents represented a motivated group, with more successful academic backgrounds than other groups of undergraduate students with disabilities. These factors all limit the generalizability of the findings.

Second, the measures used in this study were based exclusively on self-report. Despite the ensured anonymity of respondents, social desirability, defensiveness, and other reactive confounds may have influenced participants' responses. Self-report instruments are also subjective in the sense that they are based upon attitudinal and behavioral data provided by the subjects rather than objective data (e.g., actual GPA, as identified in the student record) or informed proxies (i.e., family members, peers, 
faculty).

Third, the exclusive reliance on web-based survey methodology poses its own set of unique challenges and limitations. In the current study, this primarily included the possibility of measurement errors in translating a survey from traditional paper-and-pencil format to an electronic survey format.

Fourth, this study used a correlational design. Therefore, no proven causal inferences can be made concerning directionality of relations between predictors (i.e., college stress, functionality, engagement coping, perceived social support) and adaptation-associated criterion measures of college adjustment (i.e., life satisfaction, academic performance, and psychosocial-emotional adjustment to college). It is conceivable that college adjustment outcomes (i.e., life satisfaction, academic performance, and psychosocial-emotional adjustment to college) may influence degree of college stress, functionality, engagement coping, and perceived social support. Moreover, in a correlational design, temporal relations among the study variables cannot be established.

Fifth, the Disability-Functional Limitations Scale (i.e., DFLS) was developed by the researcher to assess the degree or level of disability-related restriction(s) in the ability to perform everyday activities and roles pertaining to daily life as a college student. That is to say, before the current study, there was no known established measure of functionality pertaining to college students with a wide variety of physical, cognitive/neurocognitive and psychiatric disabilities. Measuring functionality with a not 
formally validated instrument introduced unknown error variance into the regression analyses.

Sixth, the current study used a measurement of perceived social support (i.e., SSA-R; Vaux et al., 1986) that could be conceived as too global, and therefore, not sensitive enough to detect higher order interaction effects. Moreover, the SSA-R was only able to capture one function of social support; that of esteem support.

Seventh, this study relied on parametric statistics that assume interval-level measurement (i.e., Pearson correlation coefficients) to analyze data that included ordinal-level measurement (e.g., cumulative GPA and Brief COPE Inventory's scoring systems). Although this practice is not uncommon among researchers in the field of rehabilitation psychology, the potential for statistical distortions must be recognized.

Notable limitations of the current study were also related to sample size and statistical power. More specifically, these limitations included: (a) the relatively modest sample size (i.e., $N=103$ ), diluting statistical power to detect significant effects, thus increasing the probability of Type II error; (b) the many statistical tests at .05 alpha level increasing the probability of Type I error; and (c) the relatively modest sample size limiting the generalizability of findings. Such factors could result in spurious findings. Post-hoc power analysis however, revealed that for (a) seven predictors, $R^{2}=.15, \alpha=.05, n=103$; power $=.87$; (b) seven predictors, $R^{2}=.10, \alpha=.05, n=103$; power $=.65 ;$ and (c) seven predictors, $R^{2}=.15, \alpha=.01 ; n=103$; power $=.69$. Thus, the application of more restrictive guidelines resulted in power that was still moderate to 
good.

In the context of the current study, academic performance (i.e., GPA-S) and life satisfaction (i.e., SWLS) were both crude/nonspecific measures of adaptation-associated college adjustment. As an academic-related outcome variable, the GPA-S was too global, and other potential contributing variables (e.g., motivation, intelligence) should be considered. The SWLS was also too global to capture how well a student adapts to the demands of college life. Moreover, there are many other life areas that contribute to quality-of-life and life satisfaction. For example, QoL has been empirically related to experiences of self-mastery (e.g., Rosenfeld, 1992; Zautra \& Goodhart, 1979), suggesting that self-efficacy may be one of the most significant predictors of life satisfaction (Andrews \& Withey, 1976; Cicerone \& Azulay, 2007).

It is also important to note that recruitment efforts for this study were far more arduous than had been anticipated at study conception. First of all, the potential recruitment pool of first-year and second year undergraduate students with disabilities was unknown. As a result, it was not possible to determine the current study's response rate. Secondly, the initial e-mail announcement inviting volunteers to participate in the study yielded a response of only thirty-five usable surveys. Originally, a sample of about 300 first-year and second-year students with CID, from three universities (i.e., about 100 students from each university), were expected to participate in the study. In order to obtain an adequate sample size, data were collected during winter term 2008, spring term 2008, summer term 2008, and fall term, 2008). A factor that may have 
negatively influenced volunteer participation was the relatively large number of response items (i.e., 168 items) included in the study.

\section{Research Recommendations}

In consideration of the above noted limitations, it is recommended that future research: (a) improve statistical rigor by securing a larger sample size and/or reduce level of significance from .05 (i.e., $p<.05$ ) to a more conservative .01 (i.e., $p<.01)$ level; (b) improve generalizability of the findings by recruiting a more heterogeneous group of respondents; (c) use a longitudinal design to establish causal relations among measures of college stress, functional limitations, engagement coping, perceived social support, and adaptation-associated outcome measures of college adjustment (i.e., life satisfaction, academic performance, psychosocial-emotional adjustment to college); (d) use a measure of perceived social support that captures several different typologies of support functions (i.e., ISEL; Cohen \& Hoberman, 1983); (e) use proxy-reporting measures (i.e., peers, family, instructors); (f) explore the contribution of other potential predictor variables (e.g., locus of control, self-efficacy, optimism, hope, extroversion, neuroticism); (g) examine the contribution of other socio-demographic variables (e.g., ethnicity, socio-economic status); (h) explore the contribution of other disability-related variables as predictors (e.g., stigma, visibility of disability); and, (i) establish validity and reliability of the DFLS through future studies replicating its use.

Although the current study supported the role of engagement-type coping as a 
predictor of college adjustment, very little is known about the possible beneficial role of disengagement-type strategies in coping and adjustment to college among students with disabilities. Both qualitative and quantitative research would contribute to a broader and more in-depth understanding of disengagement-type coping strategies, and under what circumstances (if any) specific strategies may be linked to successful coping and adjustment to college among students with disabilities.

Lastly, it should be noted that until a more substantial body of research accumulates, it will be difficult to draw definitive and more fine-grained conclusions regarding the study variables (e.g., college stress; functionality; engagement coping; disengagement coping; perceived social support) and their relationship to college adjustment.

\section{Implications for Rehabilitation Practice}

Several important clinical implications can be drawn, especially if the findings of this study are replicated. First, if engagement-oriented coping strategies are indeed an effective modality for improved college adjustment (as measured by life satisfaction) in the face of disability-related functional limitations, then cognitive-behavioral strategies designed to promote active, goal-oriented and problem-directed components should be implemented (Kennedy \& Duff, 2001; King \& Kennedy, 1999; Livneh \& Cook, 2005; Taylor, 2006). One such program that has empirical support in terms of helping individuals with CID develop the requisite coping skills with which to manage their functional status is called Coping Effectiveness Training (CET) (Keefe et al., 1997, 
2004; Kennedy \& Duff, 2001; Kennedy, Lowe, Grey, \& Short, 1995; Kennedy, Evans, \& Sandhu, 2009; King \& Kennedy, 1999). Originally developed by Kennedy and colleagues (Kennedy \& Duff, 2001; King \& Kennedy, 1999; Kennedy et al., 2009) as an approach to facilitating adaptive coping in individuals with spinal cord injury, CET is now employed as a treatment intervention for clients with a broad variety of chronic illness and disability conditions (e.g., Folkman et al., 1991; Keefe et al., 1997, 2004). The five major phases of the CET program include: (a) appraisal skills development (learning how to identify causes of stress as well as stress-triggering events), (b) stress analysis and breakdown (identifying situations that elicit the stress; breaking down complex/global stress into specific stressors), (c) realistic coping (evaluating stressful situations as a loss, harm, possible threat, or challenge for gain or growth);

(d) changeability examination (distinguishing between malleable and immutable aspects of stressors) and, (e) adaptive coping implementation (training application of particular coping strategies to specific stressors, and training to increase effectiveness in selecting and maintaining support resources).

Second, if successful adaptation to college among first-year and second-year undergraduate students with disabilities is directly associated with an engagement coping orientation and a perceived supportive social context, then counseling professionals can promote interventions for their clients that include: (a) an engagement-focused coping modality, and (b) a social support modality.

Engagement-oriented coping modality. As noted by Ramsay and Rostain (2006), 
these adaptive coping interventions usually involve a cognitive-behavioral approach. It is first helpful for the counseling professional to assist students in setting realistic goals. In a college setting, the goals are typically focused on academic issues, such as earning good grades. Although it may be understandable for a students' goal to earn an " $\mathrm{A}$ " in a class, such an outcome is more than can be reasonably promised. On the other hand, the student can be encouraged to focus on behavioral goals that will increase the likelihood of earning a good grade. Such goals might include improving class attendance, making use of academic support services, and completing assigned readings prior to each class.

Social support modality. Peer-led support groups have empirical support as a highly effective and low cost modality for reducing and managing stress, and ultimately, promoting overall adjustment to college. (Chen \& Katz, 2009; Gray, Vitak, Easton, \& Ellison, 2013; Mattanah et al., 2010). This modality offers a mutual, empathic environment where students with disabilities are encouraged to share their experiences, thoughts, and feelings in facing and overcoming challenges related to college life experiences. For example, Mattanah et al. (2010) implemented a 9-week, 90-minute per-session, peer led social support intervention for first-year college students that included semi-structured activities pertaining to: (a) creating new social ties; (b) balancing work, academics, and a social life; (c) peer pressures, values, and college life; (d) residential issues; (e) expectations versus realities of college life; and (f) examining old social ties. Each group meeting included a brief check-in, a group discussion, and a wrap-up period. During some sessions, group members were also 
given information about campus resources (e.g. student service center [for academic

support], writing center, counseling center) that they could use for further support. Each group included 6-10 students randomly assigned to participate in intervention $(n=88)$ or control $(n=83)$ peer support groups. The control condition received one session during the 9-week intervention period. First-year college students participating in the intervention group reported reduced loneliness, and a significantly greater level of perceived social support following the intervention than did students in the control group. Intervention participants were provided with the opportunity to become more involved with the campus community by coming to a weekly support group where they developed meaningful, relatively "deep" connections with fellow students and felt supported by sharing common adjustment struggles. An open-ended response from an intervention participant captures the feeling of mutual support:

The transition group was an incredible opportunity for those of us able to participate. I feel that all students, incoming freshmen at least, should be given the opportunity to participate in these small groups of 10 people or less. If it had not been for the transition group I would not be as successful and as social as I have been. (Mattanah et al., 2010, p. 98).

More than specific content, the most salient feature of the peer-led support group was in facilitating intimate exchanges among participating students. According to Mattanah et al. (2010), this feature alone provided the supportive social context first-year students found valuable to ease their transition and adjustment to college.

\section{Conclusion}

Because engagement coping and perceived social support appear to exert direct 
effects on college adjustment, counseling efforts by rehabilitation practitioners should address both. Moreover, engagement coping strategies may also act to mitigate the impact of disability-related functional limitations on college adjustment. Ultimately, intervention programs should be directed towards helping lower division students with disabilities effectively cope with the associated stressors of college life. 


\section{References}

Adams, K. S., \& Proctor, B. E. (2010). Adaptation to college for students with and without disabilities: Group differences and predictors. Journal of Postsecondary Education and Disability, 22, 166-184.

Achenbach, T. M. (1997). Manual for the young adult self-report and young adult behavior checklist. Burlington, VT: University of Vermont Department of Psychiatry.

Adreon, D., \& Durocher, J. S. (2007). Evaluating the college transition needs of individuals with high-functioning Autism Spectrum Disorders. Intervention in School and Clinic, 42, 271-279.

Aiken, L. S., \& West, S. G. (1991). Multiple regression: Testing and interpreting interactions. Thousand Oaks, CA: Sage Publishing.

Aldwin, C., \& Revenson, T. A. (1987). Does coping help? A reexamination of the relation between coping and mental health. Journal of Personality and Social Psychology, 53, 337-348.

Allgower, A., Wardle, J., \& Steptoe, A. (2001). Depressive symptoms, social support, and personal health behaviors in young men and women. Health Psychology, 20 (3), 223-227.

American Psychiatric Association (2000). Diagnostic and statistical manual of mental disorders (4th ed.), Washington, DC: Author. 
Andrews, F. M., \& Withey, S. B. (1976). Social indicators of well-being: Americans' Perceptions of Life Quality. New York, NY.

Antonak, R. F., \& Livneh, H. (1992). A review of research on psychosocial adjustment to impairment among persons with epilepsy. Journal of Epilepsy, 5, 194-205.

Antonak, R. F., \& Livneh, H. (1995). Psychosocial adaptation to disability and its investigation among persons with multiple sclerosis. Social Science \& Medicine, $40(8), 1099-1108$.

Appelhans, B. M., \& Schmeck, R. R. (2002). Learning styles and approach versus avoidant coping during academic exam preparation. College Student Journal, 36 $(1), 1-5$.

Arnett, J. J. (2000). Emerging adulthood: A theory of development from the late teen through the twenties. American Psychologist, 55, 469-480.

Aspinwall, L. G, \& Taylor, S. E. (1992). Modeling cognitive adaptation: A longitudinal investigation of the impact of individual differences and coping on college adjustment and performance. Journal of Personality and Social Psychology, 63 (6), 989-1003.

Baker, S. R. (2003). A prospective longitudinal investigation of social problem-solving appraisals on adjustment to university, stress, health, and academic motivation and performance. Personality and Individual Differences, 35, 569-591.

Baker, R. W., \& Schultz, K. L. (1992). Measuring expectations about college adjustment. NACADA Journal, 12, 23-32. 
Baker, R. W., \& Siryk, B. (1984). Measuring adjustment to college. Journal of Counseling Psychology, 31, 179-189.

Baker, R. W., \& Siryk, B. (1999). SACQ: Student Adaptation to College Questionnaire manual. Los Angeles, CA, Western Psychological Services.

Baron, R. M., \& Kenny, D. A. (1986). The moderator-mediator variable distinction in social psychological research: Conceptual, strategic, and statistical considerations. Journal of Personality and Social Psychology, 51, 1173-1182.

Barrera, M., Jr. (1986). Distinctions between social support concepts, measures, and models. American Journal of Community Psychology, 14, 413-445.

Bean, J. P. (1980). Dropout and turnover: The synthesis and test of a causal model of student attrition. Research in Higher Education, 12, 155-187.

Beck, A. T., \& Steer, R. A. (1993). Manual for the Beck Anxiety Inventory. San Antonio, TX: The Psychological Corporation.

Beck, A. T., Steer, R. A., \& Brown, G. K. (1996). Manual for the Beck Depression Inventory. San Antonio, TX: The Psychological Corporation.

Beck, R. Taylor, C., \& Robbins, M. (2002). Missing home? Sociotropy and autonomy and their relationship to psychological distress and homesickness in college freshmen. Anxiety, Stress, and Coping, 16 (2), 155-166.

Bean, J. P., \& Bradley, R. K. (1986). Untangling the satisfaction-performance relationship for college students. Journal of Higher Education, 57, 393-412. 
Benton, S. A., Robertson, J. M., Wen-Chih, T., Newton, F. B., \& Benton, S. L. (2003). Changes in counseling center client problems across 13 years. Professional Psychology: Research and Practice, 34 (1), 66-72.

Bewick B., Koutsopoulou, G., Miles, J., Slaa, E., \& Barkham, M. (2010). Changes in undergraduate students' psychological well-being as they progress through university. Studies in Higher Education, 35, 633-645.

Billings, A. G., \& Moos, R. H. (1981). The role of coping responses and social resources in attenuating the stress of life events. Journal of Behavioral Medicine, $4(2), 139-157$.

Billings, A. G., \& Moos, R. H. (1984). Coping, stress and social resources among adults with unipolar depression. Journal of Personality and Social Psychology, 46, 877-891.

Bishop, M. (2005). Quality of life and psychosocial adaptation to chronic illness and acquired disability: A conceptual and theoretical synthesis. Rehabilitation Counseling Bulletin, 48 (4), 219-230.

Bishop, M., Smedema, S. M., \& Lee, E. J. (2009). Outcome measurement and adjustment to chronic illness and disability: Quality of life. Understanding psychosocial adjustment to chronic illness and disability: A handbook for evidence-based practitioners in rehabilitation, 521-528. 
Blankfeld, D. G., \& Holahan, C. J. (1999). Social support, coping, and psychological adjustment among caregivers of head-injured patients. Psychology and Health, 14, 609-624.

Blase, S. L., Gilbert, A. N., Costello, E. J., Hoyle, R. H., Swartzwelder, H. S., \& Rabiner, D. L. (2009). Self-reported ADHD and adjustment in college: Cross-sectional and longitudinal findings. Journal of Attention Disorders, 13 (3), 297-309.

Bowlby, J. (1973). Attachment and loss: Vol. 2. Separation: Anxiety and anger. New York, NY: Basic Books.

Bowlby, J. (1980). Attachment and loss: Vol. 3. Loss, sadness and depression. New York, NY: Basic Books.

Breslau, J., Lane, M., Sampson, N., \& Kessler, R. C. (2008). Mental disorders and subsequent educational attainment in a US national sample. Journal of Psychiatric Research, 42 (9), 708-716.

Brissete, I., Scheier, M. F., \& Carver, C. S. (2002). The role of optimism in social network development, coping, and psychological adjustment during a life transition. Journal of Personality and Social Psychology, 82 (1), 102-111.

Brooks, J. H., \& DuBois, D. L. (1995). Individual and environmental predictors of adjustment during the first year of college. Journal of College Student Development, 36, 347-358. 
Brooks, N. A., \& Matson, R. R. (1982). Social-psychological adjustment to multiple sclerosis. Social Science and Medicine, 16, 2129-2135.

Bruyere, S. M., Van Looy, S. A., \& Peterson, D. B. (2005). The International Classification of Functioning, Disability and Health: Contemporary literature overview. Rehabilitation Psychology, 50 (2), 113-112.

Burleson, B. R., \& Mortenson, S. R. (2003). Explaining cultural differences in evaluations of emotional support behaviors: Exploring the mediating influences of value systems and interaction goals. Communication Research, 30, 113-146.

Cairney, J., Boyle, M., Offord, D. R., \& Racine, Y. (2003). Stress, social support and depression in single and married mothers. Social Psychiatry and Psychiatric Epidemiology, 38, 442-449.

Calvete, E., \& Connor-Smith, J. K. (2006). Perceived social support, coping, and symptoms of distress in American and Spanish students. Anxiety, Stress, and Coping, 19 (1), 47-65.

Carnevale, A. P., \& Desrochers, D. M. (2003). Standards for what? The economic roots of $K$-16 reform. Princeton, NJ: Educational Testing Service.

Carroll, J. M., \& Iles, J. E. (2006). An assessment of anxiety levels in dyslexic students in higher education. British Journal of Educational Psychology, 76, 651-662.

Carson, R. C., Butcher, J. N., \& Mineka, S. (1998). Abnormal psychology and modern life (10th ed.). New York: Addison, Wesley, Longman. 
Carver, C. S. (1997). You want to measure coping but your protocol's too long: Consider the Brief COPE. International Journal of Behavioral Medicine, 4 (1), 92-100.

Carver, C. S., Pozo, C., Harris, S. D., Noriega, V., Scheier, M. F., Robinson, D. S., et al. (1993). How coping mediates the effect of optimism on distress: A study of women with early stage breast cancer. Journal of Personality and Social Psychology, 65, 375-390.

Carver, C. S. (1994). Situational coping and coping dispositions in a stressful transaction. Journal of Personality and Social Psychology, 66 (1), 184-195.

Carver, C. S., Scheier, M. F., \& Weintraub, J. K. (1989). Assessing coping strategies: A theoretically based approach. Journal of Personality and Social Psychology, 56, 267-283.

Cassel, J. C. (1976). The contribution of the social environment to host resistance. American Journal of Epidemiology, 104, 107-123.

Cauce, A. M., Hannan, K., \& Sargeant, M. (1992). Life stress, social support, and locus of control during early adolescence: Interactive effects. American Journal of Community Psychology, 20 (6), 787-797.

Chalk, H. (2007). Mind over matter: Cognitive-behavioral determinants of emotional distress in multiple sclerosis patients. Psychology, Health and Medicine, 12 (5), 556-566. 
Chang, E. C. (2001). A look at the coping strategies and styles of Asian Americans: Similar and different? In C. R. Snyder (Ed.), Coping with stress: Effective people and processes (pp. 222-239). New York: Oxford University Press.

Chang, E. C., \& Strunk, D. R. (1999). Dysphoria: Relations to appraisals, coping, and adjustment. Journal of Counseling Psychology, 46 (1), 99-108.

Chao, R. C. (2012). Managing perceived stress among college students: The roles of social support and dysfunctional coping. Journal of Counseling Development, $15,5-21$.

Chase, B., Cornille, T., \& English, R. (2000). Life satisfaction among persons with spinal cord injuries. Journal of Rehabilitation, 66 (3), 14-20.

Chen, Y. F., \& Katz, J. E. (2009). Extending family to school life: College students' use of the mobile phone. International Journal of Human Computer Studies, 67, 179-191.

Chopra, P., Couper, J., \& Herman, H. (2002). The assessment of disability in patients with psychotic disorders: An application of the ICIDH-2. Australian and New Zealand Journal of Psychiatry, 36 (1), 127-132.

Chwastiak, L. A., \& Von Korff, M. (2003). Disability in depression and back pain: Evaluation of the World Health Organization Disability Assessment Schedule (WHO DAS II) in a primary care setting. Journal of Clinical Epidemiology, 56, $507-514$. 
Cicerone, K. D., \& Azulay, J. (2007). Perceived self-efficacy and life satisfaction after traumatic brain injury. The Journal of Head Trauma Rehabilitation, 22 (5), 257-266.

Cobb, S. (1976). Social support as a moderator of life stress. Psychosomatic Medicine, $38(5), 300-314$.

Coffman, D. L., \& Gilligan, T. D. (2002). Social support, stress, and self-efficacy: Effects on students' satisfaction. Journal of College Student Retention, 4 (1), 53-66.

Cohen, S., \& Hoberman, H. M. (1983). Positive events and social support as a buffer of life change stress. Journal of Applied Social Psychology, 13 (2), 99-125.

Cohen, S., \& Wills, T. A. (1985). Stress, social support, and the buffering hypothesis. Psychological Bulletin, 98 (2), 310-357

Cohn, N. (1961). Understanding the process of adjustment to disability. Journal of Rehabilitation, 27, 16-18.

Collins, M. E, Mowbray, C. T., \& Bybee, D. (1999). Measuring coping strategies in an educational intervention for individuals with psychiatric disability. Health and Social Work, 24 (4), 279-290.

Compas, B. E., Connor-Smith, J. K., Saltzman, H., Thomsen, A. H., \& Wadsworth, M. E. (2001). Coping with stress during childhood and adolescence: Problems, progress, and potential in theory and research. Psychological Bulletin, 127, 87-127. 
Compas, B., Wagner, B., Slavin, L., \& Vannatta, K. (1986). A prospective study of life events, social support, and psychological symptomatology during the transition from high school to college. American Journal of Community Psychology, 14, 241-257.

Connor-Smith, J. K., \& Compas, B. E. (2002). Vulnerability to social stress: Coping as a mediator or moderator of sociotropy and symptoms of anxiety and depression. Cognitive Therapy and Research, 26, 39-55.

Connor-Smith, J. K., \& Compas, B. E. (2004). Coping as a moderator of relations between reactivity to interpersonal stress, health status, and internalizing problems. Cognitive Therapy and Research, 28 (3), 347-368.

Connor-Smith, J. K., \& Flachsbart, C. (2007). Relations between personality and coping: A meta-analysis. Journal of Personality and Social Psychology, 93, 1080-1107.

Connor-Smith, J. K., Compas, B. E., Wadsworth, M. E., Thomsen, A. H., \& Saltzman, H. (2000). Responses to stress in adolescence: Measurement of coping and involuntary stress responses. Journal of Consulting and Clinical Psychology, 68 (6), 974-992.

Constantine, M. G., Wilton, L., \& Caldwell, L. D. (2003). The role of social support in moderating the relationship between psychological distress and willingness to seek psychological help among Black and Latino college students. Journal of College Counseling, 6, 155-165. 
Cosden, M. A., \& McNamera, J. (1997). Self-concept and perceived social support among college students with and without learning disabilities. Learning Disability Quarterly, 20, 2-12.

Costa, P. T., Somerfield, M. R., \& McCrae, R. R. (1996). Personality and coping: A reconceptualization. In M. Zeidner \& N. S. Endler (Eds.), Handbook of coping: Theory, research, applications (pp. 44-61). New York, NY: John Wiley \& Sons.

Coyne, J. C., \& Racioppo, M. W. (2000). Never the twain shall meet? Closing the gap between coping research and clinical intervention research. American Psychologist, 55, 655-664.

Cowan, P. A. (1991). Individual and family life transitions: A proposal for a new definition. In P. A. Cowan \& M. Hetherington (Eds.), Family transitions (pp. 3-30). Hillsdale, NJ: Lawrence Eribaum Associates

Crespi, T. D., \& Becket, J. T. (1999). Mental health interventions for college students: Facing the family treatment crisis. Family Therapy, 26, 141-147.

Crisp, R. (1992). The long-term adjustment of 60 persons with spinal cord injury. Australian Psychologist, 27 (1), 43-47.

Crockett, L. J., Iturbide, M. I., Stone, R., Torres, A., McGinley, M., Raffaelli, M., \& Gustavo, C. (2007). Acculturative stress, social support, and coping: Relations to psychological adjustment among Mexican-American college students. Cultural Diversity and Ethnic Minority Psychology, 13 (4), 347-355. 
Cropley, M. \& Steptoe, A. (2005). Social support, life events and physical symptoms: A prospective study of chronic and recent life stress in men and women. Psychology, Health \& Medicine, 10 (4), 317-325.

Deckro, G. R., Ballinger, K. M., Hoyt, M., Wilcher, M., Dusek, J., Meyers, P., et al. (2002). The evaluation of a mind/body intervention to reduce psychological distress and perceived stress in college students. Journal of American College Health, 50 (6), 1-14.

Demakis, G. J., \& McAdams, D. P. (1994). Personality, social support and well-being among first year college students. College Student Journal, 28, 235-243.

DeNeve, K. M. (1999). Happy as an extraverted clam? The role of personality for subjective well-being. Current Directions in Psychological Science, 8 (5), $141-144$.

Derogatis, L. R. (1977). SCL-90 administration, scoring and procedures manual I for the revised version and other instruments of the Psychopathology Rating Scale Series. Baltimore, MD: Johns Hopkins.

DeStefano, T. J., Mellott, R. N., \& Patersen, J. D. (2001). A preliminary assessment of the impact of counseling on student adjustment to college. Journal of College Counseling, 4 (2), 113-121.

Devins, G. M., \& Seland, T. P. (1987). Emotional impact of multiple sclerosis: Recent findings and suggestions for future research. Psychological Bulletin, 101, 363-375. 
Diener, E. (1984). Subjective well-being. Psychological Bulletin, 95 (3), 542-575.

Diener, E., \& Fujita, F. (1995). Methodological pitfalls and solutions in satisfaction research. In M. J. Sirgy \& A. C. Samli (Eds.), New dimensions in marketing/quality-of-life research (pp. 27-46). New York, NY: Greenwood Publishing Group.

Diener, E., \& Larsen, R. J. (1993). The experience of emotional well-being.

Diener, E., \& Seligman, M. E. P. (2004). Beyond money: Toward an economy of well-being. Psychological Science in the Public Interest, 5, 1-31.

Diener, E., Emmons, R. A., Larson, R. J., \& Griffin, S. (1985). The satisfaction with life scale. Journal of Personality Assessment, 49 (1), 71-75.

Diener, E., Suh, E. M., Lucas, R. E., \& Smith, H. L. (1999). Subjective well-being: Three decades of progress. Psychological Bulletin, 12, 276-302.

Drudge, O. W., Rosen, J. C., Peyser, J. M., \& Perniadz, J. (1986). Behavioral and emotional problems and treatment in chronically brain-impaired adults. Annals of Behavioral Medicine, 8 (1), 9-14.

Dunkel-Schetter, C., \& Wortman, C. B. (1981). Dilemmas of social support: Parallels between victimization and aging. In S. B. Kiesler, J. N. Morgan, \& V. K. Oppenheimer (Eds.), Aging: Social change (pp. 349-381). New York, NY: Academic Press.

Dunn, D. S. (1996). Well-being following amputation: Salutary effects of positive meaning, optimism, and control. Rehabilitation Psychology, 41, 285-302. 
Dunn, D. S. (2005). Negotiating realities to understand others: Teaching about meaning and well-being. Journal of Social and Clinical Psychology, 24, 30-40.

Dunn, D. S. \& Dougherty, S. B. (2005). Prospects for a positive psychology of rehabilitation. Rehabilitation Psychology, 50, 305-311.

Dyson, R., \& Renk, K. (2006). Freshmen adaptation to university life: Depressive symptoms, stress, and coping. Journal of Clinical Psychology, 62 (10), $1231-1244$.

Dziegielewski, S. F., Turnage, B., \& Roest-Marti, S. (2004). Addressing stress with social work students: A controlled evaluation. Journal of Social Work Education, $40(1), 105-119$.

Edwards, J. M., \& Trimble, K. (1992). Anxiety, coping, and academic performance. Anxiety, Stress, and Coping: An International Journal, 5, 337-350.

Edwards, K. J., Herschberger, P. J., Russell, R. K., \& Market, R. J. (2001). Stress, negative social exchange, and health symptoms in university students. Journal of American College Health, 50, 75-79.

Eide, A. H., \& Roysamb, E. (2002). The relationship between level of disability, psychological problems, social activity, and social network. Rehabilitation Psychology, 47, 165-183. 
Elfstrom, M., Kennedy, P., Lude, P., \& Taylor, N. (2007). Condition-related coping strategies in persons with spinal cord lesion: A cross-national validation of the spinal cord lesion-related coping strategies questionnaire in four community samples. Spinal Cord, 45 (6), 420-428.

Elliott, T. R., Herrick, S. M., \& Witty, T. E. (1992). Problem-solving appraisal and the effects of social support among college students and persons with physical disabilities. Journal of Counseling Psychology, 39 (2), 219-226.

Endler, N. S., Kantor, L., \& Parker, J. D. A. (1994). State-trait coping, state-trait anxiety, and academic performance. Personality and Individual Differences, 16, 663-670.

Estrada, L., Dupoux, E., \& Wolman, C. (2002). The relationship between locus of control and personal-emotional adjustment and social adjustment to college life in students with and without learning disabilities. College Student Journal, 40 (1), $15-24$.

Falek, A., \& Britton, S. (1974). Phases in coping: The hypothesis and its implications. Social Biology, 21, 1-7.

Falvo, D. R. (1999). Medical and psychosocial aspects of chronic illness and disability's (2nd ed.). Gaithersburg, MD: Aspen.

Felce, D., \& Perry, J. (1995). Quality of life: Its definition and measurement. Research in Developmental Disabilities, 16 (1), 51-74. 
Felsten, G., \& Wilcox, K. (1992). Influences of stress and situation-specific mastery beliefs and satisfaction with social support on well-being and academic performance. Psychological Reports, 70, 291-303.

Ferguson, E. (2001). Personality and coping traits: A joint factor analysis. British Journal of Health Psychology, 6, 311-325.

Ferington, F. E. (1986). Personal control and coping effectiveness in spinal cord injured persons. Research in Nursing \& Health, 9 (3), 257-265.

Field, A. (2009). Discovering statistics using SPSS (3rd ed.). Thousand Oaks, CA: Sage.

Folkman, S. (1992). Improving coping assessment: Reply to Stone and Kennedy-Moore. In Friedman (Ed.), Hostility, coping, and health (pp. 215-223). Washington, DC: American Psychological Association.

Folkman, S., Chesney, M., McKusick, L., Ironson, G., \& Johnson, D. S. (1991). Translating coping theory into intervention. In Eckenrode (Ed.), The social context of coping (pp. 239-259). New York, NY: Plenum.

Folkman, S., \& Greer, S. (2000). Promoting psychological well-being in the face of serious illness: When theory, research and practice inform each other. Psycho-Oncology, 9, 11-19.

Folkman, S., \& Launier, R. (1978). Stress-related transactions between person and environment. Perspectives in Interactional Psychology, 287, 327-332. 
Folkman, S., \& Lazarus, R. S. (1980). An analysis of coping in a middle-aged community sample. Journal of Health and Social Behavior, 21, 219-239.

Folkman, S., \& Lazarus, R. S. (1985). If it changes it must be a process: A study of emotion and coping during three stages of a college examination. Journal of Personality and Social Psychology, 48 (1), 150-170.

Folkman, S., Lazarus, R. S., Dunkel-Schetter, C., DeLongis, A., \& Gruen, R. J. (1986). Dynamics of a stressful encounter: Cognitive appraisal, coping, and encounter outcomes. Journal of Personality and Social Psychology, 50 (5), 992-1001.

Folkman, S., \& Moskowitz, J. T. (2000). Positive affect and the other side of coping. American Psychologist, 55, 647-654.

Folkman, S., \& Moskowitz, J. T. (2004). Coping: pitfalls and promises. Annual Review of Psychology, 55, 745-777.

Folkman, S., Schaefer, C., \& Lazarus, R. S. (1979). Cognitive processes of stress and coping. In V. Hamilton \& D. M. Warburton (eds.), Human stress and cognition: An information processing approach. New York, NY: John Wiley and Sons.

Fram, E. H., \& Bonvillian, G. (2001). Employees as part-time students: Is stress threatening the quality of their business education? Advanced Management Journal, 66 (3), 30-35.

Frank, R. G., VanValin, P. H., \& Elliott, T. R. (1987). Adjustment to spinal cord injury: A review of empirical and non-empirical studies. Journal of Rehabilitation, 53, 43-48. 
Fransen, J., Uebelhart, D., Stucki, G., Langeneggert, T., Seitz, M., \& Michel, B. A. (2002). The ICIDH-2 as a framework for the assessment of functioning and disability in rheumatoid arthritis. Annals of Rheumatology and Disability, 61, $225-231$.

Frazier, P. A., Tix, A. P., \& Barron, K. E. (2004). Testing moderator and mediator effects in counseling psychology research. Journal of Counseling Psychology, 51, $115-131$.

Friedlander, L. J., Reid, G. J., Shupak, N., \& Cribbie, R. (2007). Social support, self-esteem, and stress as predictors of adjustment to university among first-year undergraduates. Journal of College Student Development, 48 (3), 259-274.

Frisch, M. B. (2000). Improving mental and physical health care through quality of life therapy and assessment. In E. Diener \& D. R. Rahz (Eds.), Advances in quality of life theory and research (pp. 207-241). Dordrecht, Netherlands: Kluwer Academic Press.

Frisch, M. B., Clark, M. P., Rouse, S. V., Rudd, M. D., Paweleck, J. K., Greenston, A., \& Kopplin, D. A. (2005). Predictive and treatment validity of life satisfaction and the Quality of Life Inventory. Assessment, 12, 66-78.

Gall, T. L., Evans, D. R., \& Bellerose, S. (2000). Transition to first-year university: Patterns of change in adjustment across life domains and time. Journal of Social and Clinical Psychology, 19 (4), 544-567. 
Garret, J. B. (2001). Gender differences in college related stress. Undergraduate Journal of Psychology, 14, 21-27.

Gerdes, H., \& Mallinckrodt, B. (1994). Emotional, social, and academic adjustment of college students: A longitudinal study of retention. Journal of Counseling \& Development, 72, 281-288.

Gloria, A. M., \& Ho, T. A. (2003). Environmental, social, and psychological experiences of Asian American undergraduates: Examining issues of academic persistence. Journal of Counseling \& Development, 81, 93-105.

Goldman, C. S. \& Wong, E. H. (1997). Stress and the college student. Education, 117, 604-610.

Gottesman, D., \& Lewis, M. C. (1982). Differences in crisis reactions among cancer and surgery patients. Journal of Consulting and Clinical Psychology, 50, 381-388.

Granello, D. H., \& Wheaton, J. E. (2004). Online data collection: Strategies for research. Journal of Counseling \& Development, 82 (4), 387-393.

Gregg, N. (2007). Underserved and underprepared: Postsecondary learning disabilities. Learning Disabilities Research and Practice, 22, 219-228.

Gregg, N., Hoy, C., Flaherty, D. A., Norris, P., Coleman, C., Davis, M., et al. (2005). Decoding and spelling accommodations for postsecondary students demonstrating dyslexia: It's more than processing speed. Learning Disabilities: A Contemporary Journal, 3, 1-17. 
Haden, S. C., Scarpa, A., Jones, R. T., \& Ollendick, T. H. (2007). Posttraumatic stress disorder symptoms and injury: The moderating role of perceived social support and coping for young adults. Personality and Individual Differences, 42 (7), 1187-1198.

Halamandaris, K. F., \& Power, K. G. (1999). Individual differences, social support and coping with examination stress: A study of the psychosocial and academic adjustment of first year home students. Personality and Individual Differences, 26 (4), 665-685.

Halamandaris, K. F., \& Power, K. G. (1997). Individual differences, dysfunctional attitudes, and social support: A study of the psychosocial adjustment to university life of home students. Personality and Individual Differences, 22, 93-104.

Hamarat, E., Thompson, D., Zabrucky, K., Steele, D., \& Matheny, K. (2001). Perceived stress and coping resource availability as predictors of life satisfaction in young, middle-aged, and older adults. Experimental Aging Research, 27, 181-196.

Hamilton, S. F., \& Hamilton, M. A. (2006). School, work and emerging adulthood. In J. J. Arnett \& J. L. Tanner (Eds.), Emerging adults in America: Coming of age in the $21^{\text {st }}$ century (pp. 440-473). Washington, DC: American Psychological Association.

Hammell, K. (2004). Quality of life among people with high spinal cord injury living in the community. Spinal Cord, 42 (11), 607-620. 
Hammer, B. L., Grigsby, D. T., \& Woods, S. (1998). The conflict demand of work, family, and school among students at an urban university. The Journal of Psychology, 132 (2), 220-226.

Harari, H., Jones, C., \& Sek, H. (1988). Stress syndromes and stress predictors in American and Polish college students. Journal of Cross-Cultural Psychology, 19, 243-255.

Harding, J. (2011). Financial circumstances, financial difficulties and academic achievement among first-year undergraduates. Journal of Further and Higher Education, 35, 1-17.

Hartley, M. T. (2012). Assessing and promoting resilience: An additional tool to address the increasing number of college students with psychological problems. Journal of College Counseling, 15, 37- 51.

Heiman, T., \& Kariv, D. (2004). Coping experience among students in higher education. Educational Studies, 30 (4), 441-455.

Heinemann, A. W. (2000). Functional status and quality-of-life measures. In R. G. Frank, \& T. R. Elliott (Eds.), Handbook of Rehabilitation Psychology (pp. 261-286). Washington, DC: American Psychological Association. Heinemann, A. W., \& Mallinson, T. (2010). Functional status and quality-of-life measures. In R. G. Frank, M. Rosenthal, \& B. Caplan (Eds.), Handbook of Rehabilitation Psychology (pp. 147-164). Washington, DC: American Psychological Association. 
Heller, K., \& Swindle, R. W. (1983). Social networks, perceived social support and coping with stress. In R. D. Felner, L. A. Jason, J. Moritsugu, \& S. I. Farber (Eds.), Preventive psychology: Theory, research and practice in community intervention (pp. 87-103). New York, NY: Pergamon.

Heppner, P. P., Kivlighan, D. M., Jr., \& Wampold, B. E. (2008). Research design in counseling (3rd ed.). Belmont, CA: Brooks/Cole.

Herrington, A. N., Matheny, K. B., Curlette, W. L., McCarthy, C. J., \& Penick, J. (2005). Lifestyles, coping resources, and negative life events as predictors of emotional distress in university women. The Journal of Individual Psychology, 61 (4), 343-358.

Hertel, J. B. (2002). College student generational status: Similarities, differences, and factors in college adjustment. The Psychological Record, 52, 3-18.

Hickman, G. P., Bartholomae, S., \& McKenry, P. C. (2000). Influences of parenting styles on the adjustment and academic achievement of traditional college freshmen. Journal of College Student Development, 41, 41-54.

Hinderlie, H. H., \& Kenny, M. (2002). Attachment, social suppot, and college adjustment among Black students at predominantly white universities. Journal of College Student Development, 43 (3), 327-340.

Hollahan, C., \& Moos, R. (1981). Social support and psychological distress: Longitudinal analysis. Journal of Abnormal Psychology, 90, 365-378. 
Holahan, C., \& Moos, R. (1987). Personal and contextual determinants of coping strategies. Journal of Personality and Social Psychology, 52, 946-955.

Holahan, C., Moos, R., \& Bonin, L. (1997). Social support, coping, and adjustment: A resources model. In G. Pierce, B. Lakey, I. G. Sarason, \& B. Sarason (Eds.), Sourcebook of theory and research on social support and personality (pp. 169-186). New York, NY: Plenum.

Holahan, C., Moos, R., \& Schaefer, J. A. (1996). Coping, stress resistance, and growth: Conceptualizing adaptive functioning. In M. Zeidner \& N. S. Endler (Eds.), Handbook of coping: Theory, research, applications (pp. 24-43). New York, NY: John Wiley \& Sons.

Holahan, C. J., Moos, R., Holahan, C. K., \& Brennan, P. L. (1995). Social support, coping, and depressive symptoms in a late-middle aged sample of patients reporting cardiac illness. Health Psychology, 14 (2), 152-163.

Holahan, C. J., Moos, R., Holahan, C. K., \& Brennan, P. L. (1997). Social context, coping strategies, and depressive symptoms: An expanded model with cardiac patients. Journal of Personality and Social Psychology, 72, 918-928.

Holland, K. D., \& Holahan, C. K. (2003). The relation of social support and coping to positive adaptation to breast cancer. Psychology and Health, 18 (1), 15-29. 
Holmbeck, G. N. (1997). Toward terminological, conceptual, and statistical clarity in the study of mediators and moderators: Examples from the child clinical and pediatric psychology literatures. Journal of Consulting and Clinical Psychology, $65,599-610$.

Holmbeck, G. N. (2002). Post hoc probing of significant moderational and mediational effects in studies of pediatric populations. Journal of Pediatric Psychology, 27, 87-96.

Horowitz, M. J. (1985). Disasters and psychological responses to stress. Psychiatric Annals, 15 (3), 161-167.

Hovantz, C. A., \& Kozora, E. (1989). Life stress and clinically elevated MMPI scales: Gender differences in the moderating influence of coping. Journal of Clinical Psychology, 45 (5), 766-777.

Hoyt, W. T., Leierer, S., \& Millington, M. J. (2006). Analysis and interpretation of findings using multiple regression techniques. Rehabilitation Counseling Bulletin, $49(4), 223-233$.

Huebner, E. S., Seligson, J. L., Valois, R. F., \& Suldo, S. M. (2006). A review of the Brief Multidimensional Students' Life Satisfaction Scale. Social Indicators Research, 79, 447-484.

Humphrey, R., \& McCarthy, P. (1998). Stress and the contemporary student. Higher Education Quarterly, 52, 221-242. 
Hunsberger, B., Prancer, S. M., Pratt, M., \& Alisat, S. (1994). The transition to university: Is religion related to adjustment? Research in the Social Scientific Study of Religion, 7, 81-199.

Hutchison, C. (1999). Social support: Factors to consider when designing studies that measure social support. Journal of Advanced Nursing, 29 (6), 1520-1526.

Hysenbegasi, A., Hass, S. L., Rowland, C. R. (2005). The impact of depression on academic productivity of university students. Journal of Mental Health Policy Economics, 8, 143-151.

Jackson, L. M., Pancer, S. M., Pratt, M. W., \& Hunsberger, B. E. (2000). Great expectations: The relation between expectancies and adjustment during the transition to university. Journal of Applied Social Psychology, 30, 2100-2125.

Jelsma, J. (2009). Use of the International Classification of Functioning, Disability and Health: A literature survey. Journal of Rehabilitation Medicine, 41(1), 1-12.

Julal, F. S. (2012). Use of student support services among university students: Associations with problem-focused coping, experience of personal difficulty and psychological distress. British Journal of Guidance \& Counseling, 1, 1-12.

Kadison, R., \& DiGeronimo, T. F. (2004). College of the overwhelmed: The campus mental health crisis and what to do about it. San Francisco, CA: Jossey-Bass.

Kaminski, P., Turnock, P., Rosen, L., \& Laster. S. (2006). Predictors of academic success among college students with attention disorders. Journal of College Counseling, 9, 60-71. 
Kariv, D., \& Heiman, T. (2005). Task-oriented versus emotion-oriented coping strategies: The case of college students. College Student Journal, 39 (1), 72-84.

Keefe, F. J., Affleck, G., Lefebvre, J. C., Starr, K., \& Caldwell, D. J. (1977). Pain coping strategies and coping efficacy in rheumatoid arthritis: A daily process analysis. Pain, 69, 35-42.

Keefe, F. J., Blumenthal, J., Baucom, D., Affleck, G., \& Waugh, R. (2004). Effects of spouse-assisted coping skills training and exercise training in patients with osteoarthritic knee pain: A randomized controlled study. Pain 110, 539-549.

Kendall, E., \& Buys, N. (1998). An integrated model of psychosocial adjustment following acquired disability. Journal of Rehabilitation, 64 (3), 16-20.

Kennedy, P., \& Duff, J. (2001). Posttraumatic stress disorder and spinal cord injuries. Spinal Cord, 39 (1), 1-10.

Kennedy, P., Evans, M., \& Sandhu, N. (2009). Psychological adjustment to spinal cord injury: The contribution of coping, hope and cognitive appraisals. Psychology, Health and Medicine, 14, 17-33.

Kennedy, P., Lowe, R., Grey, N., \& Short, E. (1995). Traumatic spinal cord injury and psychological impact: A cross-sectional analysis of coping strategies. British Journal of Clinical Psychology, 34, 627-639.

Kerr, S., Johnson, V. K., Gans, S. E., \& Krumrine (2004). Predicting adjustment during the transition to college: Alexithymia, perceived stress, and psychological symptoms. Journal of College Student Development, 45 (6), 593-611. 
Kim, J., Chatterjee, S., \& Kim, J. E. (2012). Outstanding AFCPE conference paper: Debt burden of young adults in the United States. Journal of Financial Counseling and Planning, 23 (2), 55-67.

King, C., \& Kennedy, P. (1999). Coping effectiveness training for people with spinal cord injury: Preliminary results of a controlled trial. British Journal of Clinical Psychology, 38, 5-14.

Kitzrow, M. A. (2003). The mental health needs of today's college students:

Challenges and recommendations. NASPA Journal, 41 (1), 165-179.

Koeske, G. F., \& Koeske, R. D. (1991). Underestimation of social support buffering. Journal of Applied Behavioral Science, 27 (4), 475-489.

Kolchakian, M. R., \& Sears, S. F. (1999). Religious coping in college students. Journal of Religion and Health, 38 (2), 115-125.

Krause, J., \& Crew, N. (1990). Concurrent and long-term prediction of self-reported problems following spinal cord injury. International Journal of Paraplegia, 28, 186-202.

Kubler-Ross, E. (1969). On death and dying. New York: Macmillan.

Lamothe, D., Currie, F., Alisat, S., Sullivan, T., Pratt, M., Pancer, S. M., \& Hunsberger, B. (1995). Impact of a social support intervention on the transition to university. Canadian Journal of Community Mental Health, 14, 167-180.

Lauterbach, D., \& Vrana, S. (1996). Three studies on the reliability and validity of a self-reported measure of posttraumatic stress disorder. Assessment, 3 (1), 17-25. 
Lavigne, J. V., \& Faier-Routman, J. (1992). Psychological adjustment to pediatric physical disorders: A meta-analytic review. Journal of Pediatric Psychology, 17, 133-157.

Lavigne, J. V., \& Faier-Routman, J. (1993). Correlates of psychological adjustment to pediatric physical disorders: A meta-analytic review and comparison with existing modes. Journal of Developmental and Behavioral Pediatrics, 14 (2), $117-123$.

Lazarus, R. S. (1999). Stress and coping: A new synthesis. New York, NY: Springer.

Lazarus, R. S. (2000). Toward better research on stress and coping. American Psychologist, 55 (6), 665-673.

Lazarus, R. S., \& Folkman, S. (1984). Stress, appraisal, and coping. New York, NY: Springer.

Lazarus, R. S., \& Launier, R. (1978). Stress-related transactions between persons and environment. In L. A. Pervin \& M. Lewis (Eds.), Perspectives in interactional psychology (pp. 287-327). New York: Plenum.

Lecompte, D. (1986). Emotional distress in a first-year university population. Acta Psychiatrica Belgica, 86, 64-70.

Lee, B. J. (2007). Moderating effects of religious/spiritual coping in the relation between perceived stress and psychological well-being. Pastoral Psychology, 55 (6), 751-759. 
Lent, R. W., Brown, S. D., Talleyrand, R., McPartland, E. B., Davis, T., Chopra, S. B. et al. (2002). Career choice barriers, supports, and coping strategies: College students' experiences. Journal of Vocational Behavior, 60, 61-72.

Lenz, B. (2001). The transition from adolescence to young adulthood: A theoretical perspective. The Journal of School Nursing, 17 (6), 300-306.

Leong, F., Bonz, M. H., \& Zachar, P. (1997). Coping styles as predictors of college adjustment among freshmen. Counseling Psychology Quarterly, 10 (2), 211-220.

Lewis, R., \& Frydenberg, E. (2004). Thriving, surviving, or going under. Which coping strategies are related to which outcomes? In E. Frydenberg (Ed.), Thriving, surviving, or going under: Coping with everyday lives (pp. 3-23). Greenwich, CT: Information Age.

Licitra-Kleckler, D. M., \& Waas, G. A. (1993). Perceived social support among high stress adolescents: The role of peers and family. Journal of Adolescent Research, $8,381-402$.

Lightsey, O. R., \& Hulsey, C. D. (2002). Impulsivity, coping, stress, and problem gambling among university students. Journal of Counseling Psychology, 49, 202-211.

Lipowski, Z. J. (1970). Physical illness, the individual and the coping process. International Journal of Psychiatry in Medicine, 1, 91-102. 
Littlefield, C. H., Rodin, G. M., Murray, M. A., \& Craven, J. L. (1990). Influence of functional impairment and social support on depressive symptoms in persons with diabetes. Health Psychology, 9, 737-749.

Livneh, H. (1986). A unified approach to existing models of adaptation to disability-II. Intervention strategies. Journal of Applied Rehabilitation Counseling, 17 (2), 6-10.

Livneh, H. (2001). Psychosocial adaptation to chronic illness and disability: A conceptual framework. Rehabilitation Counseling Bulletin, 44 (3), 151-160.

Livneh, H. \& Antonak, R. F. (1997). Psychosocial adaptation to chronic illness and disability. Gaithersburg, MD: Aspen.

Livneh, H. \& Antonak, R. F. (2005). Psychosocial adaptation to chronic illness and disability: A primer for counselors. Journal of Counseling \& Development, 83 (1), 12-20.

Livneh, H., Antonak, R. F., \& Gerhardt, J. (1999). Psychosocial adaptation to amputation: The role of socio-demographic variables, disability-related factors and coping strategies. International Journal of Rehabilitation Research, 22 (1), 21-31.

Livneh, H., \& Cook, D. (2005). Psychosocial impact of disability. In R. M. Parker, E. M. Szymanski, \& J. B. Patterson (Eds.). Rehabilitation Counseling: Basics and beyond (pp. 187-224). Austin, TX: Pro-Ed. 
Livneh, H., \& Martz, E. (2012). Adjustment to chronic illness and disability: Theoretical perspectives, empirical findings, and unresolved issues. In P. Kennedy (Ed.). The Oxford handbook of rehabilitation psychology (pp. 47-87). New York, NY: Oxford University Press.

Livneh, H., \& Parker, R. M. (2005). Psychological adaptation to disability: Perspectives from chaos and complexity theory. Rehabilitation Counseling Bulletin, 49 (1), 17-28.

Livneh, H., \& Wilson, L. M. (2003). Coping strategies as predictors and mediators of disability-related variables and psychosocial adaptation: An exploratory investigation. Rehabilitation Counseling Bulletin, 46 (4), 194-208.

Lopez, K. G., Mauricio, A. M., Gormley, B., Simko, T., \& Berger, E. (2001). Adult attachment orientations and college student distress: The mediating role of coping styles. Journal of Counseling and Development, 79 (4), 459-464.

Lovett, B. J., \& Lewandowski, L. J. (2006). Gifted students with learning disabilities: Who are they? Journal of Learning Disabilities, 39, 515-527.

Macan, T. H., Shahani, C., Dipboye, R. L., \& Phillips, A. P. (1990). College students' time management: Correlations with academic performance and stress. Journal of Educational Psychology, 82 (4), 760-792.

Mallinckrodt, B. (1988). Student retention, social support, and dropout intention: Comparison of black and white students. Journal of College Student Development, 29, 60-64. 
Manago, A. M., Taylor, T., \& Greenfield, P. M. (2012). Me and my 400 friends: The anatomy of college students' Facebook networks, their communication patterns, and well-being. Developmental Psychology, 10, 1-12.

Mann, S. L., Ostroff, J., Winkel, G., Grana, G., \& Fox, K. (2005). Partner unsupportive responses, avoidant coping, and distress among women with early breast cancer: Patient and partner perspectives. Health Psychology, 24, 635-641.

Martz, E. (2004). A one-year follow-up study on psychosocial adaptation of college students with disabilities. Journal of Vocational Rehabilitation, 21, 1-7.

Mattanah, J. F., Ayers, J. F., Brand, B. L., Brooks, L. J., Quimby, J. L., \& McNary, S. W. (2010). A social support intervention to ease the college transition: Exploring main effects and moderators. Journal of College Student Development, 51, 93-108.

McCabe, M. P., McKern, S., \& McDonald, E. (2004). Coping and psychological adjustment among people with multiple sclerosis. Journal of Psychosomatic Research, 56, 355-361.

McFatter, R. M. (1994). Interactives in predicting mood from extraversion and neuroticism. Journal of Personality and Social Psychology, 66 (3), 570-578.

McKenzie, K., \& Schweitzer, R. (2001). Who succeeds at university? Factors predicting academic performance in first year Australian university students. Higher Education Reserch \& Development, 20 (1), 21-33. 
McNulty, K., Livneh, H., \& Wilson, L. M. (2004). Perceived uncertainty, spiritual well-being, and psychosocial adpatation in individuals with multiple sclerosis. Rehabilitation Psychology, 49 (2), 91-99.

Megivern, D., Pellerito, S., \& Mowbray, C. (2003). Barriers to higher education for individuals with psychiatric disabilities. Psychiatric Rehabilitation Journal, 26 (3), 217-232.

Mena, F. J., Padilla, A. M., \& Maldonado, M. (1987). Acculturative stress and specific coping strategies among immigrant and later generation college students. Hispanic Journal of Behavioral Sciences, 9, 207-225.

Michalos, A. C., \& Orlando, A. (2006). A note on student quality of life. Social Indicators Research, 79, 51-59.

Miech, R. A., \& Shanahan, M. J. (2000). Socioeconomic status and depression over the life course. Journal of Health and Social Behavior, 41 (2), 162-176.

Milsom, A. (2007). Interventions to assist students with disabilities through school transitions. Professional School Counseling, 10 (3), 273-278.

Milsom, A., \& Hartley, M. T. (2005). Assisting students with learning disabilities transitioning to college: What school counselors should know. Professional School Counseling, 8 (5), 436.

Misra, R. (2000). Academic stress of college students: Comparison of student and faculty perceptions. College Student Journal 
Misra, R., \& Castillo, L. G. (2004). Academic stress among college students.

Comparison of American and international students. International Journal of Stress Management, 11, 132-148.

Misra, R., McKean, M. (2000). College students' academic stress and its relation to their anxiety, time management, and leisure satisfaction. American Journal of Health Studies, 16 (1), 41-51.

Mitchell, R. E., Billings, A. G., \& Moos, R. H. (1983). Social support and well-being: Implications for prevention programs. Journal of Primary Prevention, 3, 77-98.

Mooney, S. P., Sherman, M. S., \& LoPresto, C. T. (1991). Academic locus of control, self-esteem, and perceived distance from home as predictors of college adjustment. Journal of Counseling and Development, 69, 445-448.

Moos, R. H. (1977). Coping with physical illness. New York, NY: Plenum.

Moos, R. H. (1993). Coping Responses Inventory Adult Form Professional Manual. Odessa, FL: Psychological Assessment Resources.

Moos, R. H. \& Holahan, C. J. (2007). Adaptive tasks and methods of coping with illness and disability. In E. Martz \& H. Livneh (Eds.), Coping with chronic illness and disability: Theoretical, empirical, and clinical aspects (pp. 107-126). New York, NY: Springer.

Moos, R. H., \& Holahan, C. J. (2003). Dispositional and contextual perspectives on coping: Toward an integrative framework. Journal of Clinical Psychology, 59, $1387-1403$. 
Moos, R. H., \& Schaefer, J. A. (1984). The crisis of physical illness. In R. H. Moos (Ed.), Coping with physical illness. Vol. 2: New perspectives (pp. 3-31). New York, NY: Plenum.

Moos, R., \& Schaefer, J. (1986). Coping with life crises: An integrated approach. New York, NY: Plenum Press.

Moos, R. H., \& Schaefer, J. A. (1993). Coping resources and processes: Current concepts and measures. In Goldberger, L., \& Breznitz, S. (Eds.), Handbook of stress: Theoretical and clinical aspects (2nd ed.). New York, NY: Free Press.

Morosanu, L., Handley, K., \& O’Donovan, B. (2010). Seeking support: Researching first-year students' experiences of coping with academic life. Higher Education Research \& Development, 29 (6), 665-678.

Morrison, R., \& O’Connor, R. C. (2004). Predicting psychological distress in college students: The role of rumination and stress. Journal of Clinical Psychology, 61, $1-14$.

Mortenson, S. T. (2006). Cultural differences and similarities in seeking social support as a response to academic failure: A comparison of American and Chinese college students. Communications Education, 55 (2), 127-146.

Murray, C., Lombardi, Bender, \& Gerdes (2012). Social support: Main and moderating effects on the relation between financial stress and adjustment among college students with disabilities. Social Psychology of Education, 16 (2), 277-295. 
Neilson, J. A. (2001). Successful university students with learning disabilities. Journal of College Student Psychotherapy, 15 (4), 37-48.

Nelson, M. C., Lust, K., Story, M., \& Ehlinger, E. (2008). Credit card debt, stress and key health risk behaviors among college students. American Journal of Health Promotion, 22, 400-407.

Nicholas, K. R., Menchetti, B. M., \& Nettles, S. M. (2005). An exploratory investigation of structured writing strategy training for African-American college students with learning disabilities. Journal of College Reading and Learning, 36, $37-59$.

Norwalk, K., Norvilitis, J. M., \& MacLean, M. G. (2009). ADHD symptomatology and its relationship to factors associated with college adjustment. Journal of Attention Disorders, 13 (3), 251-258.

Norvillitis, J. M., Sun, L., \& Zhang, J. (2009). ADHD symptomatology and adjustment to college in China and the United States. Journal of Learning Disabilities, 43 (1), 86-94.

Nunnally, J. C. (1978). Psychometric theory. New York, NY: McGraw-Hill.

Oakland, S., Ostell, A. (1996). Measuring coping: A review and critique. Human Relations, 49 (2), 133-155.

Olff, M., Brosschot, J. F., \& Godsert, G. (1993). Coping styles and health. Personality and Individual Differences, 15, 81-90. 
O’Reilly, B. K. (1995). The Social Support Appraisals Scale: Construct validation for psychiatric inpatients. Journal of Clinical Psychology, 51 (1), 37-42.

Pancer, S. M., Hunsberger, B., Pratt, M. W., \& Alisat, S. (2000). Cognitive complexity of expectations and adjustment to university in the first year. Journal of Adolescent Research, 15, 38-57.

Park, C. L. (2005). Religion as a meaning-making framework in coping with life stress. Journal of Social Issues, 61 (4) 707-729.

Parker, R. M., Schaller, J., \& Hansmann, S. (2003). Catastrophe, chaos, and complexity models and psychosocial adjustment to disability. Rehabilitation Counseling Bulletin, 46 (4), 234-241.

Parkes, C. M. (1975). Psychosocial transitions: Comparison between reactions to loss of a limb and loss of a spouse. British Journal of Psychiatry, 127, 204-210.

Pavot, W., \& Diener, E. (1993). Review of the satisfaction with life scale. Psychological Assessment, 5, 164-172.

Pavot, W., Diener, E., Colvin, C. R., \& Sandvik, E. (1991). Further validation of the satisfaction with life scales: Evidence for the cross-method convergence of well-being measures. Journal of Personality Assessment, 57 (1), 149-161.

Pealer, L. N., Weiler, R. M., Pigg, R. M., \& Dorman, S. M. (2001). The feasibility of a web-based surveillance system to collect risk behavior data from college students. Health Education \& Behavior, 28, 547-559. 
Pearlin, L, I., \& Schooler, C. (1978). The structure of coping. Journal of Health and Social Behavior, 19, 2-21.

Pearlin, L. I., Menaghan, E. G., Lieberman, M. A., \& Mullan, J. T. (1981). The stress process. Journal of Health and Social Behavior, 19, 2-21.

Pengilly, J. W., \& Dowd, E. T. (2000). Hardiness and social support as moderators of stress. Journal of Clinical Psychology, 56, 813-820.

Pierce, G. R., Lakey, B., Sarason, I. G., \& Sarason, B. R. (1997). Source-book of social support and personality. New York, NY: Plenum Press.

Pierceall, E. A., \& Keim, M. C. (2007). Stress and coping strategies among community college students. Community College Journal of Research and Practice, 31, 703-712.

Pledger, C. (2003). Discourse on disability and rehabilitation issues: Opportunities for psychology. American Psychologist, 58 (4), 279-284.

Pollard, C., \& Kennedy, P. (2007). A longitudinal analysis of emotional impact, coping strategies and post-traumatic psychological growth following spinal cord injury: a 10-year review. British Journal of Health Psychology, 12 (Pt. 3), 347-362.

Pritchard, M., \& Wilson, G. (2003). Using emotional and social factors to predict student success. Journal of College Student Development, 44, 18-28.

Procidano, M. E., \& Heller, K. (1983). Measures of perceived social support from friends and from family: Three validation studies. American Journal of Community Psychology, 11, 1-24. 
Ptacek, J. T., \& Pierce, G. R. (2003). Issues in the study of stress and coping in rehabilitation settings. Rehabilitation Psychology, 48 (2), 113-124.

Radloff, L. S. (1977). The CES-D scale: A self-report depression scale for research in general population. Applied Psychological Measurement, 1, 385-401.

Radnitz, C. L. (2001). Cognitive behavioral therapy for persons with disabilities. Northvale, NJ: Jason Aronson.

Rainie, L. (2010). Internet, broadband, and cell phone statistics. Pew Research Center, $1-16$.

Ramsay, J. R., \& Rostain, A. L. (2006). Cognitive behavior therapy for college students with attention-deficit/hyperactivity disorder. Journal of College Student Psychotherapy, 21 (1), 3-20.

Raue, K., \& Lewis, L. (2011). Students with disabilities at degree-granting postsecondary institutions (NCES 2011-018). U.S. Department of Education, National Center for Education Statistics, Washington, DC: U.S. Government Printing Office.

Reifman, A., \& Dunkel-Schetter, C. (1990). Stress, structural social support, and well-being in university students. Journal of American College Health, 38, 271-277.

Rodriguez, N., Mira, C. B., Myers, H. F. Morris, J. K., \& Cardoza, D. (2003). Family or friends: Who plays a greater supportive role for Latino college students? Cultural Diversity and Ethnic Minority Psychology, 9 (3), 236-250. 
Roger, D., Jarvis, G., \& Najarian, B. (1993). Detachment and coping: The construction and validation of a new scale for measuring coping strategies. Personality and Individual Differences, 15, 619-626.

Rook, K. S. (1984). The negative side of social interaction: Impact on psychological well-being. Journal of Personality and Social Psychology, 46 (5), 1097-1108.

Rosenfield, S. (1992). Factors contributing to the subjective quality of life of the chronic mentally ill. Journal of Health and Social Behavior, 33, 299-315.

Ross, S. E., Neibling, B. C., \& Heckert, T. M. (1999). Sources of stress among college students. College Student Journal, 33 (2), 312-317.

Russell, R. A. (1981). Concepts of adjustment to disability: An overview. Rehabilitation Literature, 42, 330-338.

Russell, R. K., \& Petrie, R. A. (1992). Academic adjustment of college students: Assessment and counseling. In S. D. Brown \& R. W. Lent (Eds.), Handbook of counseling psychology (2nd ed., pp. 485-511). New York, NY: Wiley.

Saber, J., Mohmoud, J. S. R., Staten, R. T., Hall, L. A., \& Lennie, T. A. (2012). The relationship among young adult college students' depression, anxiety, stress, demographics, life satisfaction, and coping styles. Issues in Mental Health Nursing, 33 (3), 149-156.

Sanders, K. S., \& DuBois, D. L. (1996). Individual and socio-environmental predictors of adjustment to college among students with disabilities. Journal of Postsecondary Education and Disability, 12 (2), 28-42. 
Sarason, I. G., \& Sarason, B. R. (2009). Social support: Mapping the construct. Journal of Social and Personal Relationships, 26, 113-120.

Sarason, I. G., Johnson, J. H., \& Siegel, J. M. (1978). Assessing the impact of life changes: Development of the Life Experiences Survey. Journal of Consulting and Clinical Psychology, 46, 932-946.

Sarason, I. G., Levine, R. B., Basham, R. B., \& Sarason, B. R. (1983). Assessing social support: The Social Support Questionnaire. Journal of Personality and Social Psychology, 44, 127-139.

Sarason, B. R., Shearin, E. N., Pierce, G. R., \& Sarason, I. G. (1987). Interrelations of social support measures. Theoretical and practical implications. Journal of Personality and Social Psychology, 52, 813-832.

Scarinci, I., C., Ames, S. C., \& Brantley, P. J. (1999). Chronic minor stressors and major life events experienced by low-income patients attending primary care clinics: A longitudinal examination. Journal of Behavioral Medicine, 22, $143-156$.

Schalock, R. L. (2004). The emerging disability paradigm and its implications for policy and practice. Journal of Disability Policy Studies, 14 (4), 204-215.

Schalock, R. L., Lemanowicz, J. A., Conroy, J. W., \& Feinstein, C. S. (1994). A multivariate investigative study of the correlates of quality of life. Journal of Developmental Disabilities, 3 (2), 59-73. 
Sharkin, B. (2004). College counseling and student retention: Research findings and implications for counseling centers. Journal of College Counseling, 7, 99-108.

Scheier, M. F., Weintraub, J. K., \& Carver, C. S. (1986). Coping with stress: Divergent strategies of optimists and pessimists. Journal of Personality and Social Psychology, 51, 1257-1264.

Schlossberg, N. K. (1981). A model for analyzing human adaptation to transition. The Counseling Psychologist, 9, 2-18.

Schnider, K. R., Elhai, J. D., \& Gray, M. J. (2007). Coping style use predicts post-traumatic stress and complicated grief symptom severity among college students reporting a traumatic loss. Journal of Counseling Psychology, 54 (3), 344-350.

Schnider, M. E., \& Ward, D. J. (2003). The role of ethnic identification and perceived support in Latinos' adjustment to college. Hispanic Journal of Behavioral Sciences, 25, 539-554.

Schultz, R. \& Decker, S. (1985). Long-term adjustment to physical disability: The role of social support, perceived control, and self-blame. Journal of Personality and Social Psychology, 48 (5), 1162-1172.

Scofield, M. E., Pape, D. A., McCracken, N., \& Maki, D. R. (1980). An ecological model for promoting acceptance of disability. Journal of Applied Rehabilitation Counseling, 11, 183-187. 
Sek, H. (1991). Life stress in various domains and perceived effectiveness of social support. Polish Psychological Bulletin, 22, 151-161.

Seligson, J. L., Huebner, E. S., \& Valois, R. F. (2005). Validation of a brief life satisfaction scale with elementary school students. Social Indicators Research, 73, $355-374$.

Selye, H. (1936). A syndrome produced by diverse nocuous agents. Nature, 138, 32.

Schalock, R. L. (2004). The emerging disability paradigm and its implications for policy and practice. Journal of Disability Policy Studies, 14 (4), 204-215.

Seligman, M. E. P., Steen, T. A., Park, N., \& Peterson, C. (2005). Positive psychology progress: Empirical validation of interventions. American Psychologist, 60 (5), $410-421$.

Sharkin, B. S. (2004). College counseling and student retention: Research findings and implications for counseling centers. Journal of College Counseling, 7 (2), 99-108.

Sharoff, K. (2004). Coping skills therapy for managing chronic and terminal illness. New York, NY: Springer Publishing.

Shaw-Zirt, B., Popali-Lehane, L., Chaplin, W., \& Bergman, A. (2005). Adjustment, social skills, and self-esteem in college students with symptoms of ADHD. Journal of Attention Disorders, 8, 109-120.

Shontz, F. C. (1965). Reactions of crisis. Volta Review, 67, 364-370.

Shontz, F. C. (1975). The psychological aspects of physical illness and disability. New York, NY: Macmillian. 
Shontz, F. C. (1982). Adaptation to chronic illness and disability. In T. Millon, C. Green, \& R. Meagher (Eds.), Handbook of clinical health psychology (pp. 153-172). New York, NY: Plenum Press.

Shontz, F. C. (2003). Rehabilitation psychology, then and now. Rehabilitation Counseling Bulletin, 46 (3), 176-181.

Silver, R. C., \& Wortman, C. B. (1984). Coping with undesirable life events. In J. Garber \& M. E. P. Seligman (Eds.), Human helplessness: Theory and application (pp. 279-340). New York, NY: Academic Press.

Simmons, J. A., Kalichman, S., \& Santrock, J. W. (1994). Human adjustment. Madison, WI: Brown \& Benchmark.

Skinner, M. E., \& Lindstrom, (2003). Bridging the gap between high school and college: Strategies for the successful transition of students with learning disabilities. Preventing School Failure, 47, 132-138.

Smart, J. (2001). Disability, society, and the individual. Austin, TX: Pro-ed.

Solberg, V., Gusavec, N., Hamann, T., Felch, J., Johnson, J., \& Lamborn, S. (1998). The Adaptive Success Identity Plan (ASIP): A career intervention for college students. The Career Development Quarterly, 47, 48-95.

Solberg, V., Hale, J. B., Villarreal, P., \& Kavanagh, J. (1993). Development of the College Stress Inventory for use with Hispanic populations: A confirmatory analytic approach. Hispanic Journal of Behavioral Sciences, 15 (4), 490-497. 
Solberg, V., \& Valdez, J., \& Villarreal, P. (1994). Social support, stress, and Hispanic college student adjustment: Test of a diathesis-stress model. Hispanic Journal of Behavioral Sciences, 16, 230-239.

Solberg, V., \& Villarreal, P. (1997). Examination of self-efficacy, social support, and stress as predictors of psychological and physical distress among Hispanic college students. Hispanic Journal of Behavioral Sciences, 19, 182-201.

Sparks, R. L., \& Lovett, B. J. (2009). College students with learning disability diagnoses: Who are they and how do they perform? Journal of Learning Disabilities, 42 (6), 494-510.

Spekman, N. J., Goldberg, R. J., \& Herman, K. L. (1992). Learning disabled children grow up: A search for factors related to success in the young adult years. Learning Disabilities Research \& Practice, 7, 161-170.

Steinhardt, M., \& Dolbier, C. (2008). Evaluation of a resilience intervention to enhance coping strategies and protective factors and decrease symptomatology. Journal of American College Health, 56 (4), 445-453.

Struthers, C. W., Perry, R. P., \& Menec, V. H. (2000). An examination of the relationship among academic stress, coping, motivation, and performance in college. Research in Higher Education, 41 (5), 581-592. 
Stucki, G., Cieza, A., \& Melvin, J. (2007). The International Classification of Functioning, Disability, and Health: A unifying model for the conceptual description of the rehabilitation strategy. Journal of Rehabilitation Medicine, 39 (4), 279-285.

Swift, A., \& Wright, M. O. D. (2000). Does social support buffer stress for college women: When and how? Journal of College Student Psychotherapy, 14 (4), 23-42.

Tabachnick, B., \& Fidell, L. S. (2012). Using multivariate statistics (6th ed.). San Francisco, CA: Pearson.

Tao, S., Dong, Q., Pratt, M. W., Hunsberger, B., \& Pancer, S. M. (2000). Social support: Relations to coping and adjustment during the transition to university in the People's Republic of China. Journal of Adolescent Research, 15 (1), 123-144.

Tate, D. G., \& Pledger, C. (2003). An integrative conceptual framework of disability: New directions for research. American Psychologist, 58 (4), 289-295

Taylor, R. (2006). Cognitive behavioral therapy for chronic illness and disability. New York, NY: Springer Verlag.

Taylor, S. E., \& Aspinwall, L. G. (1996). Mediating and moderating processes in psychological stress: Appraisal, coping, resistance, and vulnerability. In H. B. Kaplan (Ed.), Psychosocial stress: Perspectives on structure, theory, life-course, and methods (pp. 71-110). San Diego, CA: Academic Press. 
Terry, D. J. (1989). Coping resources and adaptation: Main or buffering effects? Australian Journal of Psychology, 41, 159-173.

Thoits, P. A. (1982). Conceptual, methodological, and theoretical problems in studying social support as a buffer against life stress. Journal of Health and Social Behavior, 23, 145-159.

Thoits, P. A. (1986). Social support as coping assistance. Journal of Consulting and Clinical Psychology, 54 (4), 416-423.

Thoits, P. A. (1995). Stress, coping, and social support processes: Where are we? What next? Journal of Health and Social Behavior (Extra Issue), 53-79.

Timko, C., Stovel, K. W., Baumgartner, M., \& Moos, R. H. (1995). Acute and chronic stressors, social resources, and functioning among adolescents with juvenile rheumatic disease. Journal of Research on Adolescence, 5 (3), 361-385.

Tinto, V. (1987). Leaving college. Chicago, IL: University of Chicago Press.

Tinto, V. (1993). Leaving college: Rethinking the causes and cures of student attrition. Chicago, IL: University of Chicago Press.

Tobin, D. L., Holroyd, K. A., Reynolds, R. V., \& Wigal, J. K. (1989). The hierarchical factor structure of the coping strategies inventory. Cognitive Therapy and Research, 13, 343-361.

Trieschmann, R. B. (Ed.). (1988). Spinal cord injuries: Psychological, social, and vocational rehabilitation. New York, NY: Demos Medical Publishers. 
Trockel, M. T., Barners, M. D., \& Egget, D. (2000). Health-related variables and academic performance among first-year college students: Implications for sleep and other behaviors. Journal of American College Health, 49 (3), 125-131.

Truman, M., \& Hartley, J. (1996). A comparison between the time management skills and academic performance of mature and traditional entry university students. Higher Education, 32, 199-215.

U.S. Department of Education, National Center for Education Statistics (2012). Digest of Educational Statistics (NCES 2012-001), Table 242. Retrieved from http://nces.ed.gov/programs/digest/d11/tables/dt11_242.asp

Utsey, S. O., Ponterotto, J. G., Reynolds, A. L., \& Cancelli, A. A. (2000). Racial discrimination, coping, life satisfaction, and self-esteem among African Americans. Journal of Counseling \& Development, 78, 72-80.

Valliant, G. E. (1977). Adaptation to life. Boston, MA: Little, Brown.

Valliant, G. E. (1992). Ego mechanisms of defense: A guide for clinicians and researchers. Washington, DC: American Psychiatric Press.

Van-Rooijen, L. (1986). Advanced students' adaptation to college. Higher Education, 15 (3-4), 197-209.

Vash, C. L., \& Crew, N. M. (2004). Psychology of disability (2nd ed.). New York, NY: Springer Publishing Company. 
Vaux, A., Phillips, J., Holly, L., Thomson, B., Williams, D., \& Stewart, D. (1986). The Social Support Appraisals (SS-A) scale: Studies of reliability and validity. American Journal of Community Psychology, 14 (2), 195-219.

Vogel, F. Wertheim, Vogel, G., Fresko, B. \& Wertheim, C. (2007). Peer tutoring for college students with learning disabilities: Perceptions of tutors and tutees. Journal of Learning Disabilities, 40 (6), 485-493.

Vogel, F., Holt, J. K., Sligar, S., \& Leake, E. (2008). Assessment of campus climate to enhance student success. Journal of Postsecondary Education and Disability, 21 (1), 15-31.

Vrana, S., \& Lautebach, D. (1994). Prevalence of traumatic events and post-traumatic psychological symptoms in a nonclinical sample of college students. Journal of Traumatic Stress, 7 (2), 289-302.

Wan, T. Y. (1992). Academic stress of international students attending U.S. universities. Research in Higher Education, 33 (5), 607-623.

Weller, D. J., \& Miller, P. M. (1977). Emotional reactions of patient, family, and staff in acute-care period of spinal cord injury: Part I. Social Work in Health Care, 2, 369-377.

Weyandt. L. \& DuPaul, G. (2006). ADHD in college students. Journal of Attention Disorders, 10, 9-19.

Whatley, S. L., Foreman, A. C., \& Richards, S. (1998). The relationship of coping style to dysphoria, anxiety, and anger. Psychological Reports, 83, 783-791. 
Wheaton, B. (1985). Models for the stress-buffering functions of coping resources. Journal of Health and Social Behavior, 26, 352-364.

Wilkinson, R. B., Walford, W. A., \& Espnes, G. A. (2000). Coping styles and psychological health in adolescents and young adults. A comparison of moderator and main effects models. Australian Journal of Psychology, 52 (3), 155-162.

Wilkes, S. E., \& Spivey, C. A. (2010). Resilience in undergradute social work students: Social support and adjustment to academic stress 1. Social Work Education, 29 (3), 276-288.

Wills, T., \& Fegan, M. (2001). Social networks and social support. In T. A. R. A. Baum, \& J. E. Singer (Eds.), Handbook of health psychology (pp. 139-173). Mahwah, NJ: Erlbaum.

Wineman, N. M. (1990). Adaptation to multiple sclerosis: The role of social support, functional disability and perceived uncertainty. Nursing Research, 39, 294-299.

Wineman, N. M., Durand, E. J., \& Steiner, R. P. (1994). A comparative analysis of coping behaviors in persons with multiple sclerosis or spinal cord injury. Research in Nursing and Health, 17, 185-194.

Winter, M., \& Yaffe, M. (2000). First-year students' adjustment to university life as a function of relationships with parents. Journal of Adolescent Research, 15, 9-37.

Winterowd, C. L., Street, V. L., \& Boswell, D. L. (1998). Perceived social support, disability status, and affect in college students. Journal of College Student Psychotherapy, 13 (2), 53-70. 
Wodka, E. L., \& Barakat, L. (2007). An exploratory study of the relationship of family support and coping with adjustment: Implications for college students with a chronic illness. Journal of Adolescence, 30 (3), 365-376.

Wompold, B. E., \& Freund, R. D. (1987). Use of multiple regression in counseling psychology research: A flexible data-analytic strategy. Journal of Counseling Psychology, 34 (4), 372-382.

Wolanin, T. R., \& Steele, P. E. (2004). Higher education opportunities for students with disabilities: A primer for policymakers. Washington, DC: The Institute for Higher Education Policy.

Wood-Dauphinee, S., \& Kuchler, T. (1992). Quality of life as a rehabilitation outcome: Are we missing the boat? Canadian Journal of Rehabilitation, 6 (1), 3-12.

World Health Organization (2001). World Health Organization: International Classification of Functioning, Disability and Health (ICF). Geneva, Switzerland: World Health Organization.

Wortman, C., \& Silver, R. (1989). The myths of coping with loss. Journal of Consulting and Clinical Psychology, 57 (3), 349-357.

Wright, B. A. (1983). Physical disability: A psychosocial approach (2nd ed.). New York, NY: Harper \& Row.

Wright, S. J., \& Kirby, A. (1999). Deconstructing conceptualizations of 'adjustment' to chronic illness: A proposed integrative framework. Journal of Health Psychology, 4, 259-270. 
Yagodich, N. L., Wolfe, P. S., \& Boone, R. S. (2000). Postsecondary education for individuals with multiple sclerosis: Issues and strategies. The Journal for Vocational Special Needs Education, 23 (1), 34-41.

Yalcin, I. (2011). Social support and optimism as predictors of life satisfaction of college students. International Journal for the Advancement of Counseling, 33 (2), 79-87.

Ying, W., \& Newfield, S. A. (2007). Comparing data collected by computerized and written surveys for adolescence health research. Journal of School Health, 77, 23-28.

Young, J. R. (2003, Feb. 14 ). Prozac campus. The Chronicle of Higher Education, 49 (23), A-37.

Ystgaard, M., Tambs, K., \& Dalgard, O. S. (1999). Life stress, social support and psychological distress in late adolescence: A longitudinal study. Social Psychiatry and Psychiatric Epidemiology, 34, 12-19.

Yum, J. C. K., Kember, D., \& Siaw, I. (2005). Coping mechanisms of part-time students. International Journal of Lifelong education, 24 (4), 303-317.

Zajacova, A., Lynch, \& Espenshade, T. J. (2005). Self-efficacy, stress, and academic success in college. Research in Higher Education, 46 (6), 677-694.

Zautra, A., \& Goodhart, D. (1979). Quality of life indicators: A review of the literature. Community Mental Health Review, 4, 1-10. 
Zea, M. C., Jarama, L., \& Bianchi, F. T. (1995). Social support and psychosocial competence: Explaining the adaptation to college in ethnically diverse students. American Journal of Community Psychology, 23, 509-531.

Zea, M. C., Belgrave, F. Z., Townsend, T. G., Jarama, S. L., \& Banks, R. S. (1996). The influence of social support and active coping on depression among African Americans and Latinos with disabilities. Rehabilitation Psychology, 41 (3), $225-242$.

Zimet, G. D., Dahem, N. W., Zimet, S. G., \& Farley, G. K. (1988). The multidimensional scale of perceived social support. Journal of Personality Assessment, $52(1), 30-41$.

Zivin, K., Eisenberg, D., Gollust, S. E., Golberstein, E. (2009). Persistence of mental health problems and needs in a college student population. Journal of Affective Disorders, 117 (3), 180-185.

Zullig, K. J., Huebner, E. S., \& Pun, S. M. (2009). Demographic correlates of domain-based life satisfaction reports of college students. Journal of Happiness Studies, 10, 229-238.

Zullig, K. J., Huebner, E. S., Gilman, R., Patton, J. M., \& Murray, K. A. (2005). Validation of the Brief Multidimensional Students' Life Satisfaction Scale among college students. American Journal of Health Behavior, 29, 206-214. 


\section{Appendix A}

\section{Participant Questionnaire}

Background Information. Please Indicate your response to each of the 11 items by filling in the blank or checking the appropriate response.

1. Age (please specify)

2. Please indicate your age at the time that your impairment or disability was first diagnosed:

3. Type of Disability: Please indicate the type of disability you have. Carefully read all categories before you respond.

Attention Deficit Disorder (ADD) or

Attention Deficit and Hyperactivity Disorder (ADHD)

Autism or Asperger's Syndrome

Deafness

Hard of Hearing/Hearing Impaired

Learning Disability (examples include, but are not limited to: disabilities in reading (Dyslexia), writing (Dysgraphia), or mathematics (Dyscalculia)

Visual Impairment or Blindness

Psychiatric Disability (examples include, but are not limited to: Anxiety Disorder, PTSD, Bipolar Disorder, Depression)

Mobility-Related/Orthopedic (examples include, but are not limited to: Spinal Cord Injury, Polio, Cerebral Palsy, Lower Limb Amputation) 
Health and Medical Conditions (examples include, but are not limited to: Diabetes, Epilepsy, Chronic Pain, Cancer, Chronic Fatigue Syndrome, HIV/Aids, Asthma, Chemical Sensitivity, MS)

Speech or Language Disability

Traumatic Brain Injury (TBI)

Other (please specify)

4. Racial/Ethnic Group

Black, African American

American Indian or Alaska Native

White/Caucasian, Non-Hispanic

Hispanic/Chicano/Latino

Asian or Pacific Islander

Middle Eastern

Multiple Ethnicities

Other (please specify)

5. Gender: Female __ Male 
6. Marital Status

\begin{tabular}{|c|}
\hline Single \\
\hline Divorced \\
\hline Married \\
\hline Separated \\
\hline Partnered, but not married \\
\hline Widowed \\
\hline Other (please specify) \\
\hline
\end{tabular}

7. What is your enrollment status at this college?
Full-Time Student (12 or more credits per term)
Part-Time Student (less than 12 credits per term)
No longer attending college

8. Please indicate the number of hours per week you are employed while enrolled in classes.

[ o or Only Occasional Jobs
1 to 10
11 to 20
21 to 30
31 to 40


9. Please indicate your residence while registered for classes:

\begin{tabular}{l} 
College Residence Hall/Dorm \\
College Fraternity or Sorority House \\
College Married Student Housing \\
Off-Campus Room or Apartment \\
Home of Parents or Relatives \\
Own Home or Condominium \\
\hline
\end{tabular}

10. Indicate your current, cumulative grade point average (GPA), to the best of your knowledge. Use the following scale:

_ 3.66 to 3.33 (at least a B+ average, but just below an A-)

— 3.32 to 3.00 (at least a B average, but just below a B+)

2.99 to 2.67 (at least a B- average, but just below a B)

2.66 to 2.33 (at least a C+ average, but just below a B-)

2.32 to 2.00 (at least a $\mathrm{C}$ average, but just below a $\mathrm{C}+$ )

1.99 to 1.67 (at least a C-average, but just below a C)

1.66 or below ( $\mathrm{D}$ average or below) 
11. Please indicate the college you are attending (i.e., PSU or OSU) and academic level (i.e., Freshman or Sophomore)

PSU Freshman

PSU Sophomore
OSU Freshman

OSU Sophomore 


\section{Appendix B}

\section{College Stress Inventory (CSI)}

Please use the scale below to rate your appraisal of the amount of stress you have encountered in the last month for each of the specific college experiences.

$1=$ never

$2=$ almost never

$3=$ sometimes

4 = fairly often

$5=$ very often

\section{Academic Stress}

1. __ Difficulty trying to fulfill responsibilities at home and school.

2. _ـ Difficulty taking exams.

3. __ A fear of failing to meet family expectations.

4. __ Difficulty handling your academic workload.

5. _ Difficulty writing papers.

6. Difficulty meeting deadlines for course requirements.

7. __ Difficulty because of feeling a need to perform well in school.

\section{Social Stress}

1. __ Difficulty trying to meet peers with your disability (on campus).

2. __ Difficulty finding support groups sensitive to your needs. 
3. __ Difficulty from faculty on the basis of your disability.

4. ___ Difficulty from peers on the basis of your disability

5. __ Difficulty participating in class.

6. __ Difficulty living in the local community.

7. __ Difficulty handling relationships.

8. __ Difficulty with peers treating you unlike they treat each other.

\section{Financial Stress}

1. ___ Difficulty paying student fees next quarter.

2. ___ Financial difficulties due to owing money.

3. __ Difficulty paying rent/housing.

4. ___ Difficulty paying for food/gas.

5. __ Difficulty paying for recreation and entertainment.

6. __ Difficulty due to your family experiencing money problems. 


\section{Appendix C}

\section{Disability Functional Limitations Scale (DFLS)}

Please use the scale below to rate the degree to which your ability to function within your own environment is restricted by your disability.

$1=$ not restricted at all

$2=$ only minimally restricted

$3=$ moderately restricted

$4=$ severely restricted

$5=$ totally restricted

1. __ Leisure and recreational activities

2. ___ Daily living activities (e.g., cleaning, cooking, grooming)

3. ___ Cognitive activities (e.g., reading, writing, doing math)

4. __ Meaningful relationships with others

5. __ Social interaction with others

6. Community/university involvement

7. __ Sleep/Rest 


\section{Appendix D}

\section{Social Support Appraisals-Revised (SSA-R) scale}

Below is a list of $\mathbf{2 3}$ statements about your relationships with family and friends. Please indicate how much you agree or disagree with each statement as being true.

Circle one number in each row

\begin{tabular}{|c|c|c|}
\hline $\begin{array}{l}\text { Strongly } \\
\text { Agree }\end{array}$ & Agree & Disagree \\
\hline
\end{tabular}

1. My friends respect me.

$4 \quad 3 \quad 2 \quad 1$

2. My family cares for me

$4 \quad 3 \quad 2 \quad 1$
very much.

3. I am not important

1

2

3

4

4. My family holds me

4 in high esteem.

5. I am well liked.

4

3

2

(1)

6. I can rely on my friends.

4

3

2

7. I am really admired by

4

3

2
my family.
8. I am respected by other people.

9. I am loved dearly

4

3

21
10. My friends don't care about my welfare.

11. Members of my family rely on me.

21

1

2

34

4

$\begin{array}{lll}3 & 2 & 1\end{array}$




\section{Strongly Agree Disagree Strongly}

Agree

Disagree

12. I am held in high esteem. $\quad 4 \quad 3 \quad 3 \quad 2 \quad 1$

13. I can't rely on my

$\begin{array}{llll}1 & 2 & 3 & 4\end{array}$

family for support.

14. People admire me. $\quad 4 \quad 3 \quad 3 \quad 2 \quad 1$

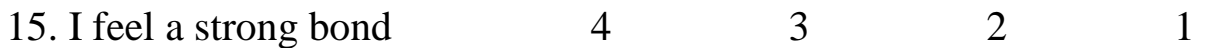

with my friends.

16. My friends look out for me.

$\begin{array}{llll}4 & 3 & 2 & 1\end{array}$

17. I feel valued by

4

3

2

1

other people.

18. My family really

4

3

$2 \quad 1$

respects me.

19. My friends and I are really important to each other.

20. I feel like I belong.

4

3

2

1

21. If I died tomorrow,

1

2

3

4 very few people would miss me.

22. I don't feel close to

1

2

3

4 members of my family.

23. My friends and I have 4 done a lot for one another. 


\section{Appendix E}

\section{Brief COPE}

INSTRUCTIONS: We are interested in how people respond when they confront difficult or stressful events in their lives. There are lots of ways to try to deal with stress. This questionnaire asks you to indicate what YOU generally do and feel, when YOU experience stressful events. Obviously, different events bring out somewhat different responses, but think about what YOU usually do when you are under a lot of stress.

Then respond to each of the following items by choosing one number for each, using the response choices listed just below. Please try to respond to each item separately in your mind from each other item. Choose your answers thoughtfully, and make your answers as true FOR YOU as you can.

Please try to answer every item. There are no "right" or "wrong" answers, so choose the most accurate answer for YOU - not what you think "most people" would say or do. Indicate what YOU usually do when YOU experience a stressful event.
1
2
3
4

I usually don't do I usually do this I usually do this I usually do

this at all a little bit a medium amount this a lot

1. I've been concentrating my efforts on doing $\quad \begin{array}{lllll}2 & 2 & 3 & 4\end{array}$ something about the situation I'm in.

2. I've been trying to come up with a strategy $\quad \begin{array}{lllll}1 & 2 & 3 & 4\end{array}$ about what to do.

3. I've been trying to see it in a different light, $\begin{array}{lllll}1 & 2 & 3 & 4\end{array}$ to make it seem more positive.

4. I've been accepting the reality of the $\quad \begin{array}{lllll}1 & 2 & 3 & 4\end{array}$ fact that it has happened.

5. I've been making jokes about it.

123



1
2
3
4

I usually don't I usually do this I usually do this I usually do do this at all a little bit a medium amount this a lot

6. I've been trying to find comfort in $\quad \begin{array}{lllll}1 & 2 & 3 & 4\end{array}$ my religion or spiritual beliefs.

7. I've been getting emotional support

$\begin{array}{llll}1 & 2 & 3 & 4\end{array}$
from others.

8. I've been trying to get advice or help

$\begin{array}{llll}1 & 2 & 3 & 4\end{array}$
from other people about what to do.

9. I've been turning to work or other $\quad \begin{array}{lllll} & 1 & 2 & 3 & 4\end{array}$

10. I've been saying to myself $\quad \begin{array}{lllll} & 1 & 2 & 3 & 4\end{array}$ "this isn't real."

11. I've been saying things to let my $\quad \begin{array}{lllll} & 2 & 3 & 3 & 4\end{array}$ unpleasant feelings escape.

12. I've been using alcohol or other

$\begin{array}{llll}1 & 2 & 3 & 4\end{array}$
drugs to make myself feel better.

13. I've been giving up trying to deal with it. $\quad \begin{array}{lllll}1 & 2 & 3 & 4\end{array}$

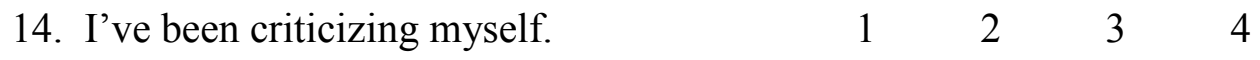

15. I've been learning to live with it. $\quad 1 \quad \begin{array}{llll} & 2 & 3 & 4\end{array}$

16. I've been taking action to try to make $\quad \begin{array}{lllll}1 & 2 & 3 & 4\end{array}$

17. I've been thinking hard about what $\quad \begin{array}{lllll}1 & 2 & 3 & 4\end{array}$ steps to take.

18. I've been looking for something good in $\quad \begin{array}{lllll}1 & 2 & 3 & 4\end{array}$ what is happening.

19. I've been making fun of the situation. $\quad \begin{array}{lllll}1 & 2 & 3 & 4\end{array}$ 
20. I've been praying or meditating.

$\begin{array}{llll}1 & 2 & 3 & 4 \\ 1 & 2 & 3 & 4\end{array}$

21. I've been getting comfort and understanding from someone.

22. I've been getting help and advice from $\quad \begin{array}{lllll}1 & 2 & 3 & 4\end{array}$ other people.

23. I've been doing something to think about $\begin{array}{llll}1 & 2 & 3 & 4\end{array}$ it less, such as going to movies, watching $\mathrm{TV}$, reading, daydreaming, sleeping, or shopping.

24. I've been refusing to believe that it 123 has happened.

25. I've been expressing my negative feelings.

$\begin{array}{lll}2 & 3 & 4\end{array}$

26. I've been using alcohol or other drugs to help me get through it.

27. I've been giving up the attempt to cope. $\quad \begin{array}{lllll}1 & 2 & 3 & 4\end{array}$

28. I've been blaming myself for things $\quad 1 \quad \begin{array}{llll}2 & 3 & 4\end{array}$ that happened. 


\section{Appendix F}

\section{Satisfaction With Life Scale (SWLS)}

Below are five statements with which you may agree or disagree. Using the 1-7 scale below, indicate your agreement with each item by placing the appropriate number on the line preceding that item. Please be open and honest in your responding. The 7-point scale is:

$1=$ strongly disagree

$2=$ disagree

3 = slightly disagree

$4=$ neither agree nor disagree

$5=$ slightly agree

$6=$ agree

$7=$ strongly agree

1. In most ways my life is close to my ideal.

2. The conditions of my life are excellent.

3. __ I am satisfied with my life.

4. So far, I have gotten the important things I want in life.

5. __ If I could live my life over, I would change almost nothing. 